\title{
Dinuclear Ce(IV) Aryloxides: Highly Active Catalysts for Anhydride/Epoxide Ring-Opening Copolymerization
}

Steven J. Gray ${ }^{\dagger}$, Karen Brown ${ }^{\dagger}$, Francis Y. T. Lam ${ }^{\dagger, \ddagger}$, Jennifer A. Garden ${ }^{\dagger}$ and Polly L. Arnold ${ }^{+, \neq, *}$

${ }^{\dagger}$ EaStCHEM School of Chemistry, The University of Edinburgh, Joseph Black Building, David Brewster Road, Edinburgh, EH9 3FJ, UK

‡ Current address: Department of Chemistry, University of California, Berkeley, Berkeley CA 94720, USA. Chemical Sciences Division, Lawrence Berkeley National Laboratory, One Cyclotron Road, Berkeley 94720, USA. e-mail: pla@berkeley.edu

\section{Contents}

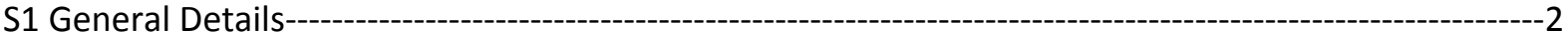

S2 Compound Syntheses --------

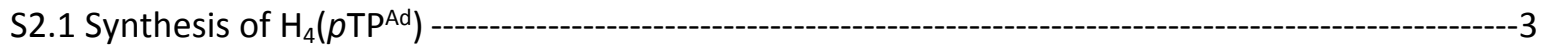

S2.2 Synthesis of $\mathrm{H}_{4}\left(m T^{A d}\right)$-------

$\mathrm{S} 2.3$ Synthesis of $\left[\mathrm{NEt}_{4}\right]_{2}\left[\mathrm{Ce}_{2} \mathrm{Cl}_{6}\left(p T P^{t}\right)\right]---$

$\mathrm{S} 2.4\left[\mathrm{NEt}_{4}\right]_{2}\left[\mathrm{Ce}_{2} \mathrm{Cl}_{2}\left(\mathrm{OSiMe}_{3}\right)_{4}\left(p \mathrm{TPt}^{t}\right)\right]--$

$\mathrm{S} 2.5\left[\mathrm{NEt}_{4}\right]_{2}\left[\mathrm{Ce}_{2} \mathrm{Cl}_{2}\left(\mathrm{OSiMe}_{3}\right)_{4}\left(m \mathrm{mPt}^{\mathrm{t}}\right)\right]$------11

$\mathrm{S} 2.6\left[\mathrm{NEt}_{4}\right]_{2}\left[\mathrm{Ce}_{2} \mathrm{Cl}_{2}\left(\mathrm{OSiMe}_{3}\right)_{4}\left(p \mathrm{pTP}^{\mathrm{Ad}}\right)\right]$

$\mathrm{S} 2.7\left[\mathrm{NEt}_{4}\right]_{2}\left[\mathrm{Ce}_{2} \mathrm{Cl}_{2}\left(\mathrm{OSiMe}_{3}\right)_{4}\left(m \mathrm{mPAd}^{\mathrm{Ad}}\right)\right]$

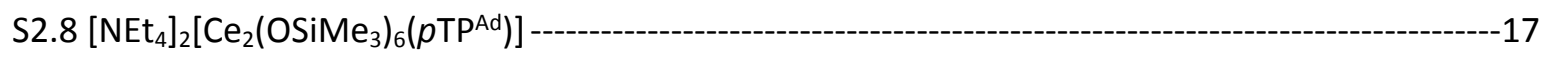

$\mathrm{S} 2.9\left[\mathrm{NEt}_{4}\right]_{2}\left[\mathrm{Ce}_{2} \mathrm{Cl}_{4}(\mathrm{ODipp})_{2}\left(p \mathrm{pTt}^{\mathrm{t}}\right)\right]$

$\mathrm{S} 2.10$ Synthesis of $\left[\mathrm{NEt}_{4}\right]_{2}\left[\mathrm{Ce}_{2} \mathrm{Cl}_{6}(m T e r T P t)\right]$

S2.11 Synthesis of neutral di-Ce(IV) complex ---

S2.12 Cyclic voltammetry studies ---

S2.13 UV-Vis ---

S3 ROCOP Protocols --

S3.1 Example procedure for $\mathrm{PA} / \mathrm{CHO}$ reactions, using $\left[\mathrm{NEt}_{4}\right]_{2}\left[\mathrm{Ce}_{2} \mathrm{Cl}_{2}\left(\mathrm{OSiMe}_{3}\right)_{4}\left(p \mathrm{PPt}^{t}\right)\right]$ as catalyst. -27

S3.2 Example procedure for ROCOP substrate scope ---------------------------------------------------31

S3.3 Rate equation determination experiments-----------------------------------------------------------35

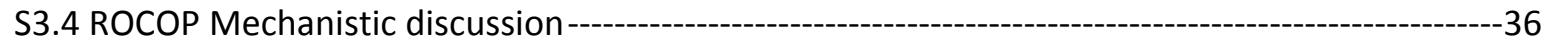

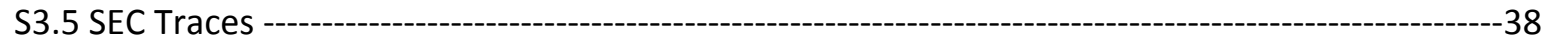

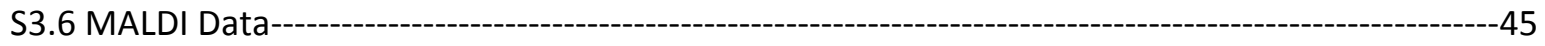

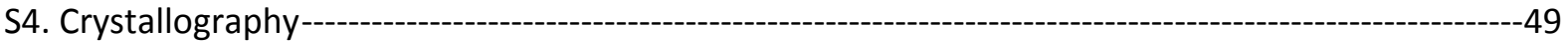

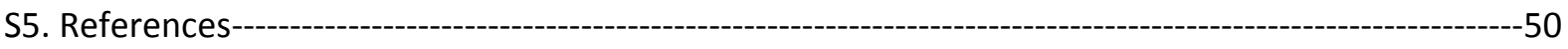




\section{S1 General Details}

All moisture and air sensitive materials were manipulated using standard high-vacuum Schlenk-line techniques and MBraun gloveboxes and stored under an atmosphere of dried and deoxygenated dinitrogen. All gases were supplied by BOC gases UK. All glassware items, cannulae and Fisherbrand $1.2 \mu \mathrm{m}$ retention glass microfibre filters were dried in a $160^{\circ} \mathrm{C}$ oven overnight before use.

Hexane, tetrahydrofuran and toluene for use with moisture and air sensitive compounds were dried using a Vac Atmospheres solvent purification system and stored over activated 4 Å molecular sieves. The solvent was cycled through a drying column containing molecular sieves for 12 hours before collection. All solvents were degassed and stored for 2 days prior to use. Benzene- $d_{6}$ and pyridine- $d_{5}$ were refluxed over potassium metal for 24 hours, freeze-pump-thaw degassed and distilled by trapto-trap distillation prior to use. All solvents were purchased from Sigma-Aldrich or Fisher Scientific.

$\mathrm{NaOSiMe}_{3}$ was sublimed prior to use and phthalic anhydride and succinic anhydride were sublimed three times prior to use. Cyclohexene oxide, $( \pm)$-propylene oxide and (+)-limonene oxide were dried over $\mathrm{CaH}_{2}$ and distilled prior to use. $\mathrm{H}_{4}\left(p \mathrm{TP}^{\mathrm{t}}\right),{ }^{1} \mathrm{H}_{4}\left(m T \mathrm{Pt}^{\mathrm{t}}\right),{ }^{1} \mathrm{H}_{4}\left(m T e r T \mathrm{Pt}^{\mathrm{t}}\right),{ }^{2}$ 2-adamantyl-p-cresol, ${ }^{3}$ $\mathrm{NaN}\left(\mathrm{SiMe}_{3}\right)_{2}{ }^{4}\left[\mathrm{NEt}_{4}\right]_{2} \mathrm{CeCl}_{6},{ }^{5}$ and $\mathrm{NaODipp}^{6,7}$ were prepared using published methods. All other chemicals were used as received.

Unless stated otherwise, all NMR spectroscopic analyses were recorded at $298 \mathrm{~K}$ using a Bruker Avance III $500.12 \mathrm{MHz}$ spectrometer with ${ }^{1} \mathrm{H}$ NMR spectra run at $500.12 \mathrm{MHz},{ }^{13} \mathrm{C}$ NMR spectra run at 126 $\mathrm{MHz}$ and ${ }^{29} \mathrm{Si}$ NMR spectra at $99.37 \mathrm{MHz}$. The ${ }^{1} \mathrm{H}$ NMR spectra were referenced internally using residual solvent signals and are reported relative to external tetramethylsilane. Chemical shifts are quoted in ppm and coupling constants in Hz. IR spectra were recorded using a Nicolet Avatar $360 \mathrm{FT}-\mathrm{IR}$ spectrometer between $4000-400 \mathrm{~cm}^{-1}$ on a powdered sample. Elemental analyses were carried out at the London Metropolitan University.

SEC analyses of the filtered polymer samples were carried out in SEC grade THF at a flow rate of 1 $\mathrm{mL}$. $\mathrm{min}^{-1}$ at $35^{\circ} \mathrm{C}$ on a 1260 Infinity $\| \mathrm{GPC} / \mathrm{SEC}$ single detection system with mixed bed C PLgel columns $\left(300 \times 7.5 \mathrm{~mm}\right.$ ). MALDI-ToF MS analyses were performed using a Bruker Daltonics UltrafleXtreme ${ }^{\mathrm{TM}}$ MALDIToF/ToF MS instrument. The sample to be analysed, dithranol matrix and KI (cationizing agent) were dissolved in THF at $10 \mathrm{mg} \cdot \mathrm{mL}^{-1}$ and the solutions were mixed in a 2:2:1 volume ratio, respectively. A droplet $(2 \mu \mathrm{L})$ of the resultant mixture was spotted on to the sample plate and submitted for MALDIToF MS analysis. 


\section{S2 Compound Syntheses}

\section{S2.1 Synthesis of $\mathrm{H}_{4}\left(p T P^{A d}\right)$}

4<smiles>Cc1ccc(O)c(C23CC4CC(CC(C4)C2)C3)c1</smiles>

0.1<smiles>O=Cc1ccc(C=O)cc1</smiles>

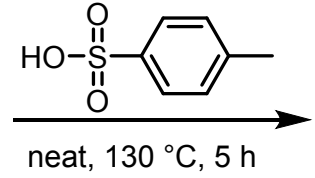

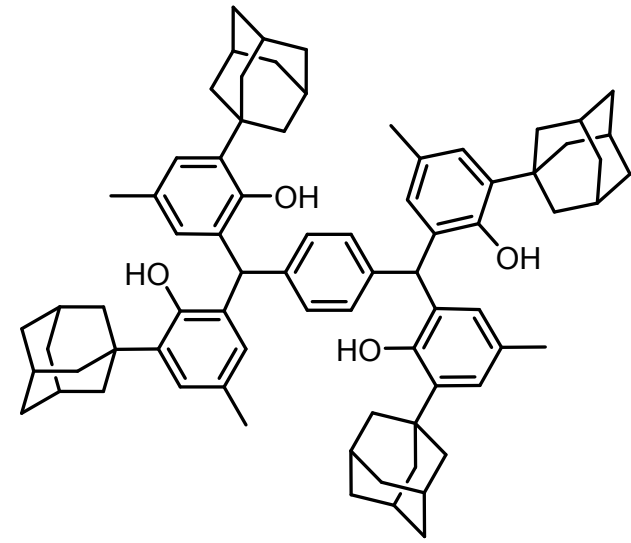

Equation S1: Synthesis of $\mathrm{H}_{4}\left(p \mathrm{pP}^{\mathrm{Ad}}\right)$.

A $250 \mathrm{~mL}$ round-bottomed flask was charged with 2-adamantyl-p-cresol (3.52 g, $14.5 \mathrm{mmol}, 4.4$ equiv.), terephthalaldehyde $(0.440 \mathrm{~g}, 3.3 \mathrm{mmol}, 1$ equiv.), $p$-toluenesulfonic acid $(0.063 \mathrm{~g}, 0.33 \mathrm{mmol}, 0.1$ equiv.) and a stir bar, the contents heated to $130{ }^{\circ} \mathrm{C}$ and stirred for 5 hours. The resulting red-orange solid was suspended in acetonitrile $\left(200 \mathrm{~cm}^{3}\right)$ and stirred with sonication until the mixture was homogenous. The light red mixture was then filtered and dried under suction, washed with boiling ethanol $\left(3 \times 50 \mathrm{~cm}^{3}\right)$ and dried under dynamic vacuum for 16 hours at $50^{\circ} \mathrm{C}$ to give the pale red/pink solid $\mathrm{H}_{4}\left(p \mathrm{PP}^{\mathrm{Ad}}\right)$. Yield: $3.81 \mathrm{~g}(82 \%)$.

${ }^{1} \mathrm{H}$ NMR $\left(500 \mathrm{MHz}\right.$, benzene- $\left.\mathrm{d}_{6}\right) \delta_{\mathrm{H}} 7.08(\mathrm{br}, 4 \mathrm{H}, \mathrm{Ar}-\mathrm{H}), 7.07(\mathrm{br}, 4 \mathrm{H}, \mathrm{Ar}-\mathrm{H}), 6.73(\mathrm{~s}, 4 \mathrm{H}, \mathrm{Ar}-\mathrm{H}), 5.63(\mathrm{~s}, 2 \mathrm{H}$, benzylic $\mathrm{C}-\mathrm{H}), 4.98(\mathrm{~s}, 4 \mathrm{H},-\mathrm{OH}), 2.19,\left(\mathrm{~m}, 24 \mathrm{H},-\mathrm{CH}_{2}\right), 2.11(\mathrm{br}, 12 \mathrm{H}), 2.02(\mathrm{br}, 12 \mathrm{H}), 1.74\left(\mathrm{~m}, 24 \mathrm{H},-\mathrm{CH}_{2}\right)$.

${ }^{13} \mathrm{C}\left\{{ }^{1} \mathrm{H}\right\} \mathrm{NMR}\left(126 \mathrm{MHz}\right.$, benzene- $\left.\mathrm{d}_{6}\right) \delta_{\mathrm{C}} 151.9(\operatorname{ArC}), 140.5(\operatorname{ArC}), 138.3(\operatorname{ArC}), 130.6(\operatorname{ArC}), 130.2(\operatorname{ArC}), 128.7(\operatorname{ArC})$, 67.8 (benzylic $\mathrm{CH}), 47.7,40.9,37.4,37.3,29.6,25.8,21.2$.

Elemental analysis $\left(\mathrm{C}_{76} \mathrm{H}_{90} \mathrm{O}_{4}\right)$ : C 85.51\%, $\mathrm{H} 8.50 \%, \mathrm{~N} 0.00 \%$ calculated; $\mathrm{C} 85.33 \%, \mathrm{H} 8.37 \%, \mathrm{~N} 0.00 \%$ found.

Figure S1: ${ }^{1} \mathrm{H}$ NMR spectrum of $\mathrm{H}_{4}\left(p T \mathrm{P}^{\mathrm{Ad}}\right)$ in $\mathrm{C}_{6} \mathrm{D}_{6}$. 


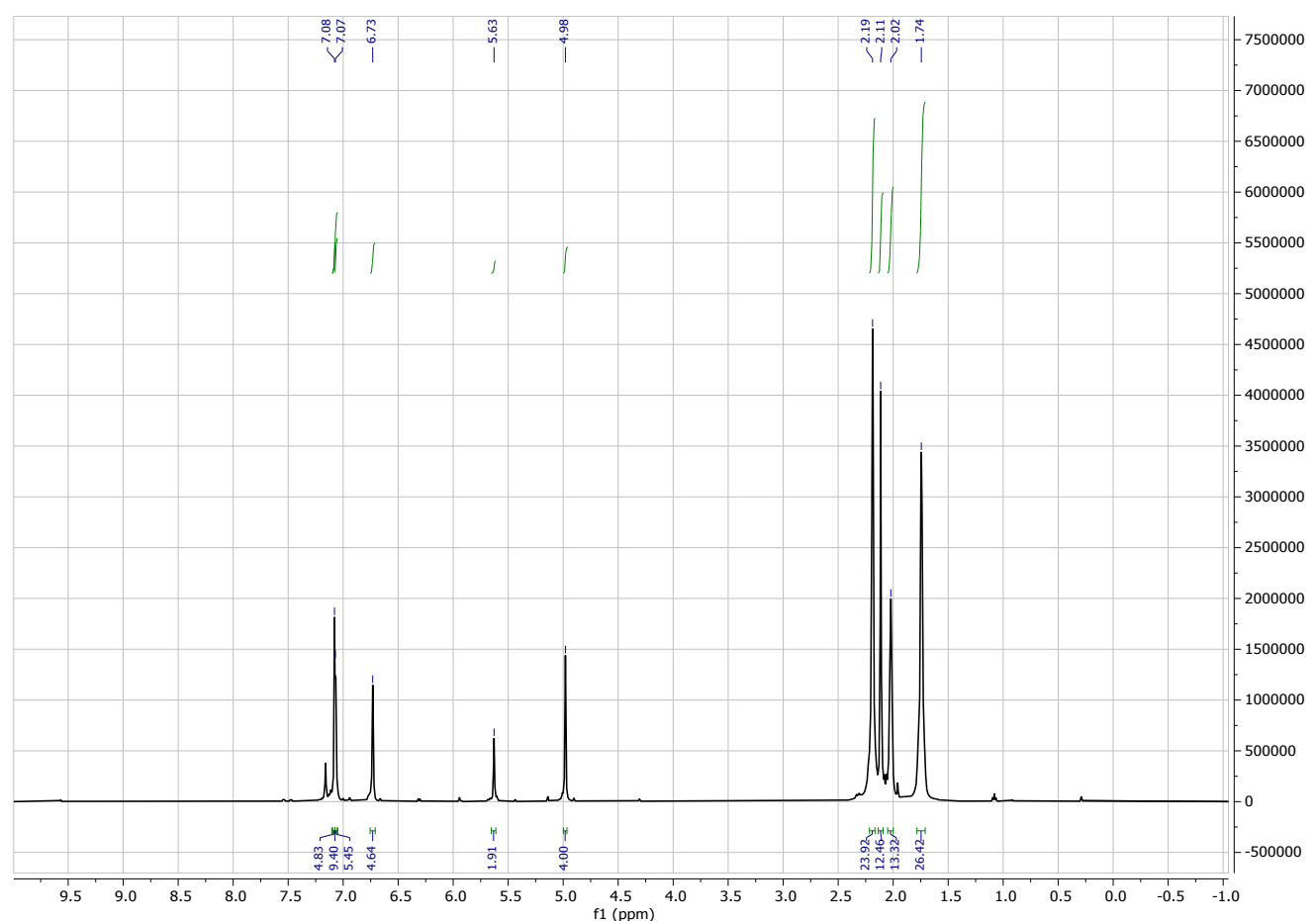

Figure S2: ${ }^{13} C\left\{{ }^{1} \mathrm{H}\right\}$ NMR spectrum of $\mathrm{H}_{4}\left(p T P^{A d}\right)$ in $\mathrm{C}_{6} \mathrm{D}_{6}$.

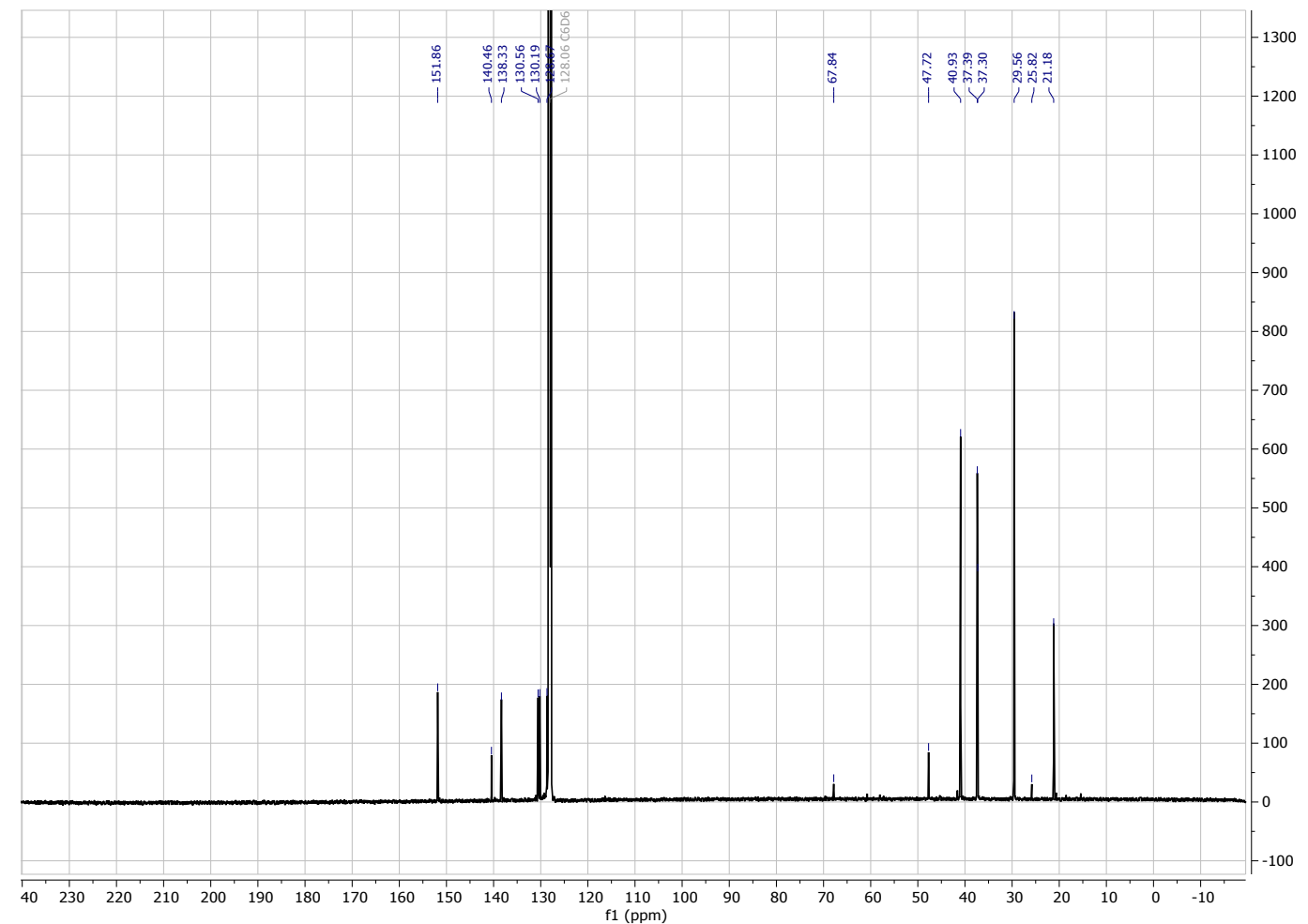

S2.2 Synthesis of $\mathrm{H}_{4}\left(m \mathrm{mP}^{\mathrm{Ad}}\right)$ 


\section{1}

4<smiles>Cc1ccc(O)c(C23CC4CC(CC(C4)C2)C3)c1</smiles><smiles>O=Cc1cccc(C=O)c1</smiles><smiles>Cc1ccc(S(=O)(=O)O)cc1</smiles><smiles>Cc1cc([Al])c(O)c(C(c2cccc(C(c3cc(C)cc([Ge])c3O)c3cc([Ge])cc([Ge])c3O)c2)c2cc(C)cc(Cl)c2O)c1</smiles>

Equation S2: Synthesis of $\mathrm{H}_{4}\left(m T^{\mathrm{Ad}}\right)$.

Synthesised by analogous procedure to $\mathrm{H}_{4}(p$ TPAd). 2-adamantyl-p-cresol ( $3.52 \mathrm{~g}, 14.5 \mathrm{mmol}, 4.4$ equiv.), isophthalaldehyde $(0.440 \mathrm{~g}, 3.3 \mathrm{mmol}, 1$ equiv.) and $p$-toluenesulfonic acid $(0.063 \mathrm{~g}, 0.33 \mathrm{mmol}, 0.1$ equiv.) were combined, heated to $130{ }^{\circ} \mathrm{C}$ and stirred for 5 hours. An identical work-up yielded $\mathrm{H}_{4}\left(\mathrm{mTP}^{\mathrm{Ad}}\right)$ as a pale-yellow solid, yield: $3.05 \mathrm{~g}(66 \%)$.

${ }^{1} \mathrm{H}$ NMR $\left(500 \mathrm{MHz}\right.$, benzene- $\left.\mathrm{d}_{6}\right) \delta_{\mathrm{H}} 7.05(\mathrm{br}, 8 \mathrm{H}, \mathrm{Ar}-\mathrm{H}), 6.71(\mathrm{br}, 4 \mathrm{H}, \mathrm{Ar}-\mathrm{H}), 5.53(\mathrm{~s}, 2 \mathrm{H}$, benzylic $\mathrm{C}-\mathrm{H}), 4.95(\mathrm{~s}, 4 \mathrm{H}$, $-\mathrm{OH}), 2.18,\left(\mathrm{~m}, 24 \mathrm{H},-\mathrm{CH}_{2}\right), 2.12(\mathrm{br}, 12 \mathrm{H}), 2.03(\mathrm{br}, 12 \mathrm{H}), 1.76\left(\mathrm{~m}, 24 \mathrm{H},-\mathrm{CH}_{2}\right)$.

${ }^{13} \mathrm{C}\left\{{ }^{1} \mathrm{H}\right\} \mathrm{NMR}\left(126 \mathrm{MHz}\right.$, benzene- $\left.\mathrm{d}_{6}\right) \delta_{\mathrm{C}} 151.8(\operatorname{ArC}), 142.1(\operatorname{ArC}), 138.3(\operatorname{ArC}), 131.2(\operatorname{ArC}), 130.2(\operatorname{ArC}), 130.0$, 128.9 (ArC), 128.5 ( $\operatorname{ArC}), 128.4(\operatorname{ArC}), 128.0(\operatorname{ArC}), 48.1,40.9,37.4,37.3,29.6,21.2$.

Elemental analysis $\left(\mathrm{C}_{76} \mathrm{H}_{90} \mathrm{O}_{4}\right): \mathrm{C} 85.51 \%, \mathrm{H} 8.50 \%, \mathrm{~N} 0.00 \%$ calculated; $\mathrm{C} 85.67 \%, \mathrm{H} 8.78 \%, \mathrm{~N} 0.00 \%$ found.

Figure S3: ${ }^{1} \mathrm{H}$ NMR spectrum of $\mathrm{H}_{4}\left(m \mathrm{mP}^{\mathrm{Ad}}\right)$ in $\mathrm{C}_{6} \mathrm{D}_{6}$.

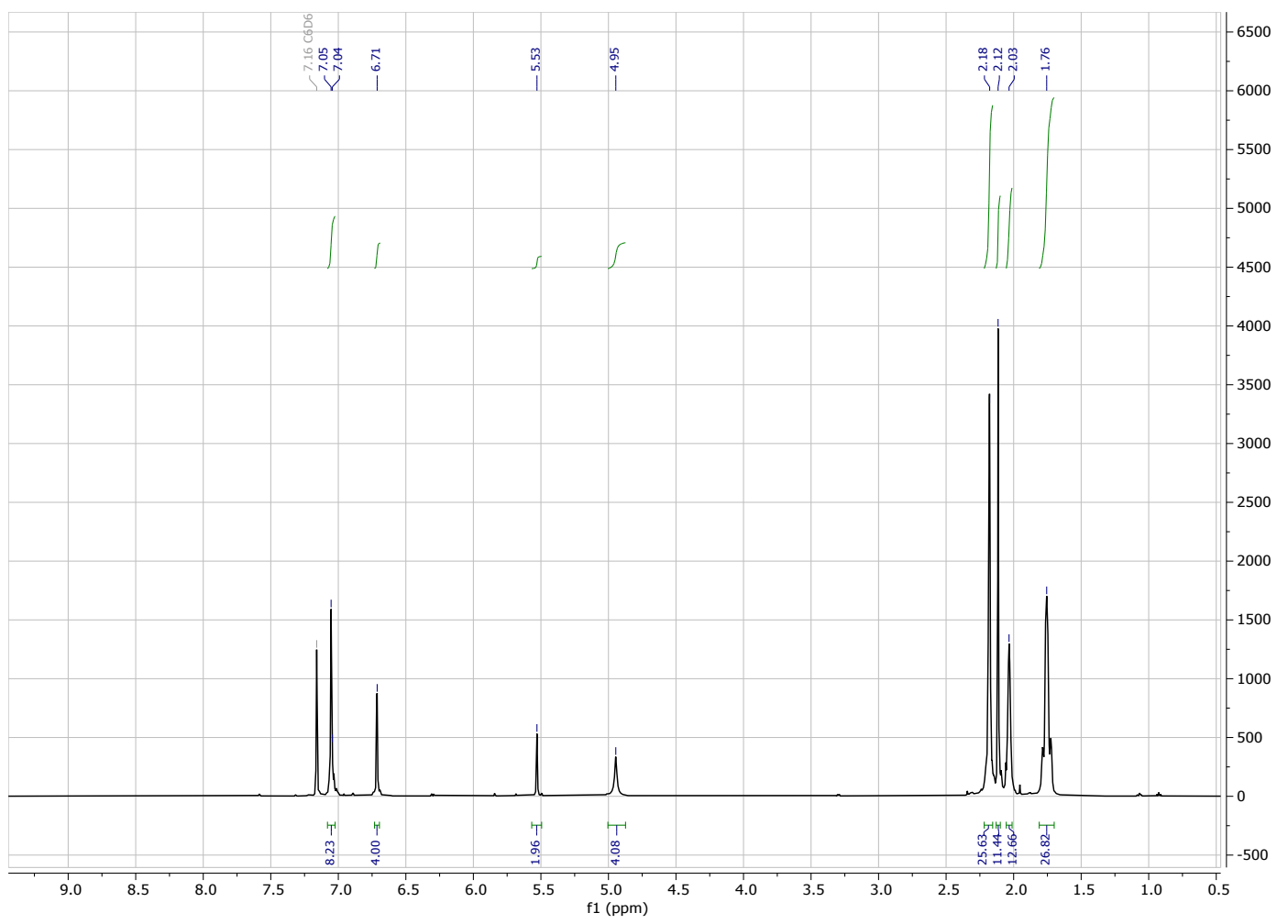

Figure S4: ${ }^{13} \mathrm{C}\left\{{ }^{1} \mathrm{H}\right\}$ NMR spectrum of $\mathrm{H}_{4}\left(m T P^{\mathrm{Ad}}\right)$ in $\mathrm{C}_{6} \mathrm{D}_{6}$. 


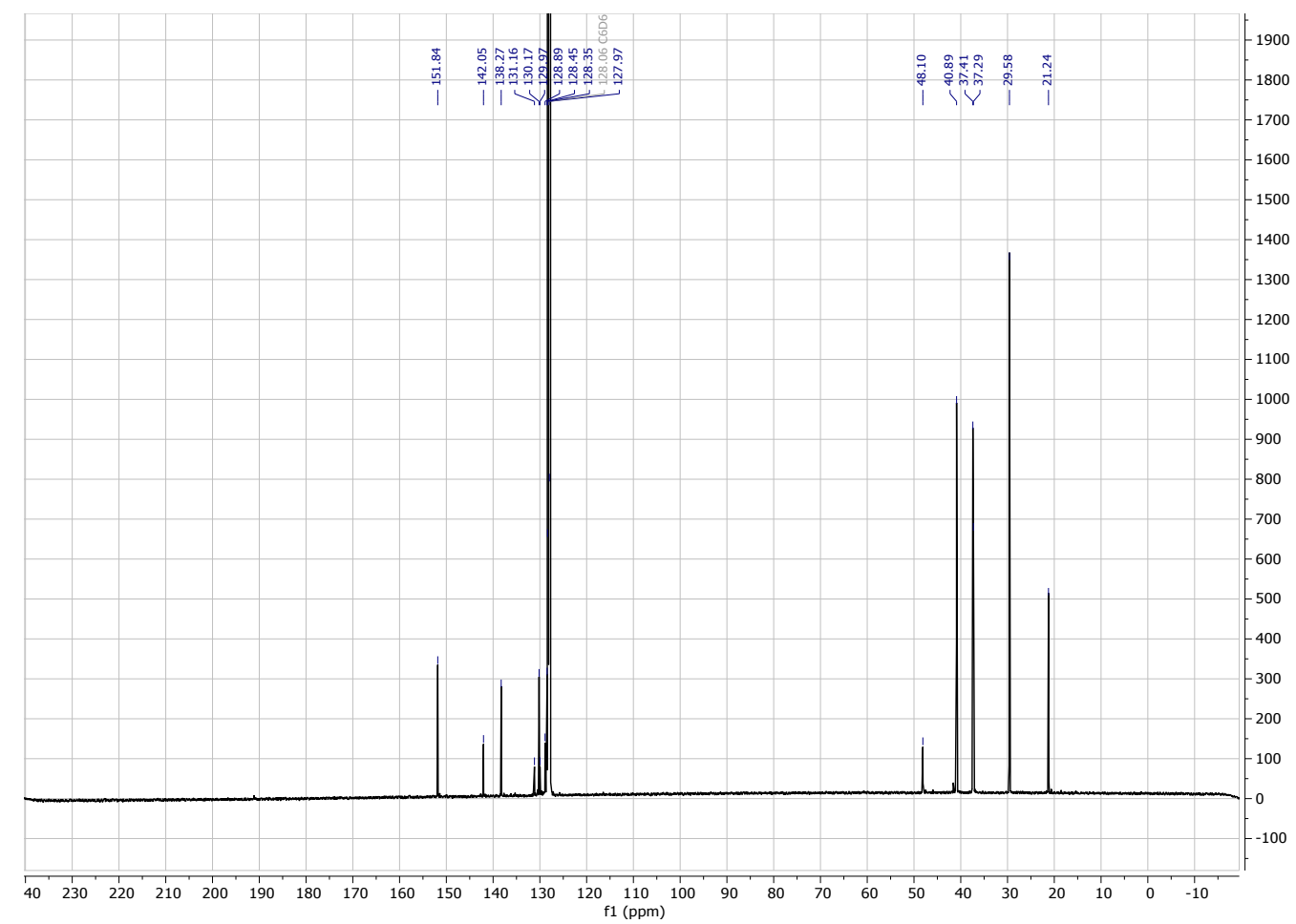

S2.3 Synthesis of $\left[\mathrm{NEt}_{4}\right]_{2}\left[\mathrm{Ce}_{2} \mathrm{Cl}_{6}\left(p \mathrm{TP}^{\mathrm{t}}\right)\right]$

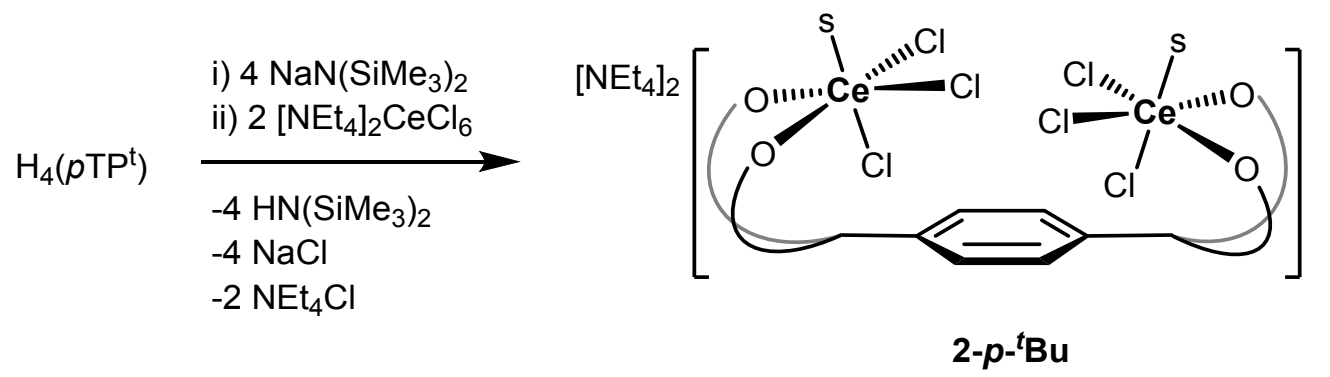

Equation S3: Synthesis of $\left[\mathrm{NEt}_{4}\right]_{2}\left[\mathrm{Ce}_{2} \mathrm{Cl}_{6}\left(p \mathrm{TPt}^{\mathrm{t}}\right)\right]$.

$\mathrm{H}_{4}\left(p \mathrm{TPt}^{\mathrm{t}}\right)\left(1.11 \mathrm{~g}, 1.20 \mathrm{mmol}, 1\right.$ equiv.) and $\mathrm{NaN}\left(\mathrm{SiMe}_{3}\right)_{2}(0.850 \mathrm{~g}, 4.90 \mathrm{mmol} 4.1$ equiv.) were dissolved in THF (25 $\mathrm{mL}$ ) and the resulting yellow-brown solution stirred for 2 hours. [ $\left.\mathrm{NEt}_{4}\right]_{2} \mathrm{CeCl}_{6}(1.55 \mathrm{~g}, 2.52 \mathrm{mmol}, 2.1$ equiv.) was suspended in THF $(15 \mathrm{~mL})$ at $0{ }^{\circ} \mathrm{C}$ and to this was slowly added the $\mathrm{Na}_{4}\left(p \mathrm{TP}^{\mathrm{t}}\right)$ solution, forming a deep purple solution which was stirred vigorously for 16 hours at room temperature. The mixture was centrifuged then cooled in an ice-bath, filtered via cannula whilst cold and the volatiles of the filtrate evaporated under reduced pressure presenting the crude product as a purple solid. This material was dried under reduced pressure then triturated with hexane $(3 \times 10 \mathrm{~mL})$ before drying again, yielding $\left[\mathrm{NEt}_{4}\right]_{2}\left[\mathrm{Ce}_{2} \mathrm{Cl}_{6}\left(p \mathrm{TP}^{\mathrm{t}}\right)\right] \mathbf{2 - \boldsymbol { p }}$ - $\mathrm{Bu}^{\mathrm{B}}(1.12 \mathrm{~g}, 56 \%)$ as a purple powder.

${ }^{1} \mathrm{H}$ NMR $\left(500 \mathrm{MHz}\right.$, pyridine- $\left.\mathrm{d}_{5}\right) \delta_{\mathrm{H}} 8.14(\mathrm{~d}, \mathrm{~J}=2.5 \mathrm{~Hz}, 2 \mathrm{H}$ Aryloxide A), 8.08 (d, J = 2.5 Hz, 2H Aryloxide B), 7.82 (s, 2H, benzyl protons), 7.60 (s, 4H, spacer), 7.48 (d, J = 2.5 Hz, 2H, Aryloxide A), 7.16 (d, J = 2.5 Hz, 2H Aryloxide B), $3.32\left(\mathrm{q}, 16 \mathrm{H},\left({ }^{+} \mathrm{N}\left(\mathrm{C}_{2} \mathrm{CH}_{3}\right)_{4}\right), 1.91\left(\mathrm{~s}, 18 \mathrm{H},{ }^{\mathrm{t}} \mathrm{Bu}\right), 1.53\left(\mathrm{~s}, 18 \mathrm{H},{ }^{\mathrm{t}} \mathrm{Bu}\right), 1.41\left(\mathrm{~s}, 18 \mathrm{H},{ }^{\mathrm{t}} \mathrm{Bu}\right), 1.40\left(\mathrm{~s}, 18 \mathrm{H},{ }^{\mathrm{t}} \mathrm{Bu}\right), 1.20(\mathrm{t}\right.$, $24 \mathrm{H},\left({ }^{+} \mathrm{N}\left(\mathrm{CH}_{2} \mathrm{CH}_{3}\right)_{4}\right)$.

${ }^{13} \mathrm{C}\left\{{ }^{1} \mathrm{H}\right\}$ NMR (126 MHz, pyridine- $\left.d_{5}\right) \delta_{\mathrm{C}} 170.0$ (q, ArC-O), 166.5 (q, ArC-O), 143.4 (q, ArC), 139.0 (q, ArC), 138.2 (q, $\operatorname{ArC}), 137.8$ (q, $\operatorname{ArC}), 134.0$ (q, ArC), 133.8 (q, ArC), 130.1 (spacer, ArC-H), 125.6 (Aryloxide B ArC-H), 124.9

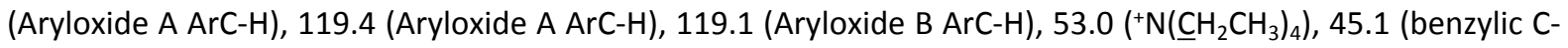


H), $36.2\left(\mathrm{q},{ }^{\mathrm{t}} \mathrm{Bu}\right), 36.1\left(\mathrm{q},{ }^{\mathrm{t}} \mathrm{Bu}\right), 35.0\left(\mathrm{q},{ }^{\mathrm{t}} \mathrm{Bu}\right), 34.8\left(\mathrm{q},{ }^{\mathrm{t}} \mathrm{Bu}\right), 33.1\left(\mathrm{CH}_{3},{ }^{\mathrm{t}} \mathrm{Bu}\right), 33.0\left(\mathrm{CH}_{3},{ }^{\mathrm{t}} \mathrm{Bu}\right), 32.5\left(\mathrm{CH}_{3},{ }^{\mathrm{t}} \mathrm{Bu}\right), 32.3\left(\mathrm{CH}_{3}\right.$, $\left.{ }^{\mathrm{t}} \mathrm{Bu}\right), 7.8\left({ }^{+} \mathrm{N}\left(\mathrm{CH}_{2} \mathrm{CH}_{3}\right)_{4}\right)$.

IR: $v_{\text {max }} / \mathrm{cm}^{-1} 2951 \mathrm{~m}, 1433 \mathrm{~m}, 1252 \mathrm{~s}, 1120,1010 \mathrm{~m}, 830 \mathrm{~s}, 530 \mathrm{~s}, 432 \mathrm{~s}$.

Elemental analysis ([NEt $\left.]_{2}\left[\mathrm{Ce}_{2} \mathrm{Cl}_{6}\left(p \mathrm{TP}^{\mathrm{t}}\right)(\mathrm{THF})_{2}\right]\right): \mathrm{C} 58.17 \%, \mathrm{H} 7.88 \%, \mathrm{~N} 1.54 \%$ calculated; $\mathrm{C} 58.22 \%, \mathrm{H} 7.41 \%, \mathrm{~N}$ $1.94 \%$ found.

Figure S5: ${ }^{1} \mathrm{H}$ NMR spectrum of $\left[\mathrm{NEt}_{4}\right]_{2}\left[\mathrm{Ce}_{2} \mathrm{Cl}_{6}\left(p T P^{\mathrm{t}}\right)\right]$ in $\mathrm{C}_{5} \mathrm{D}_{5} \mathrm{~N}$.

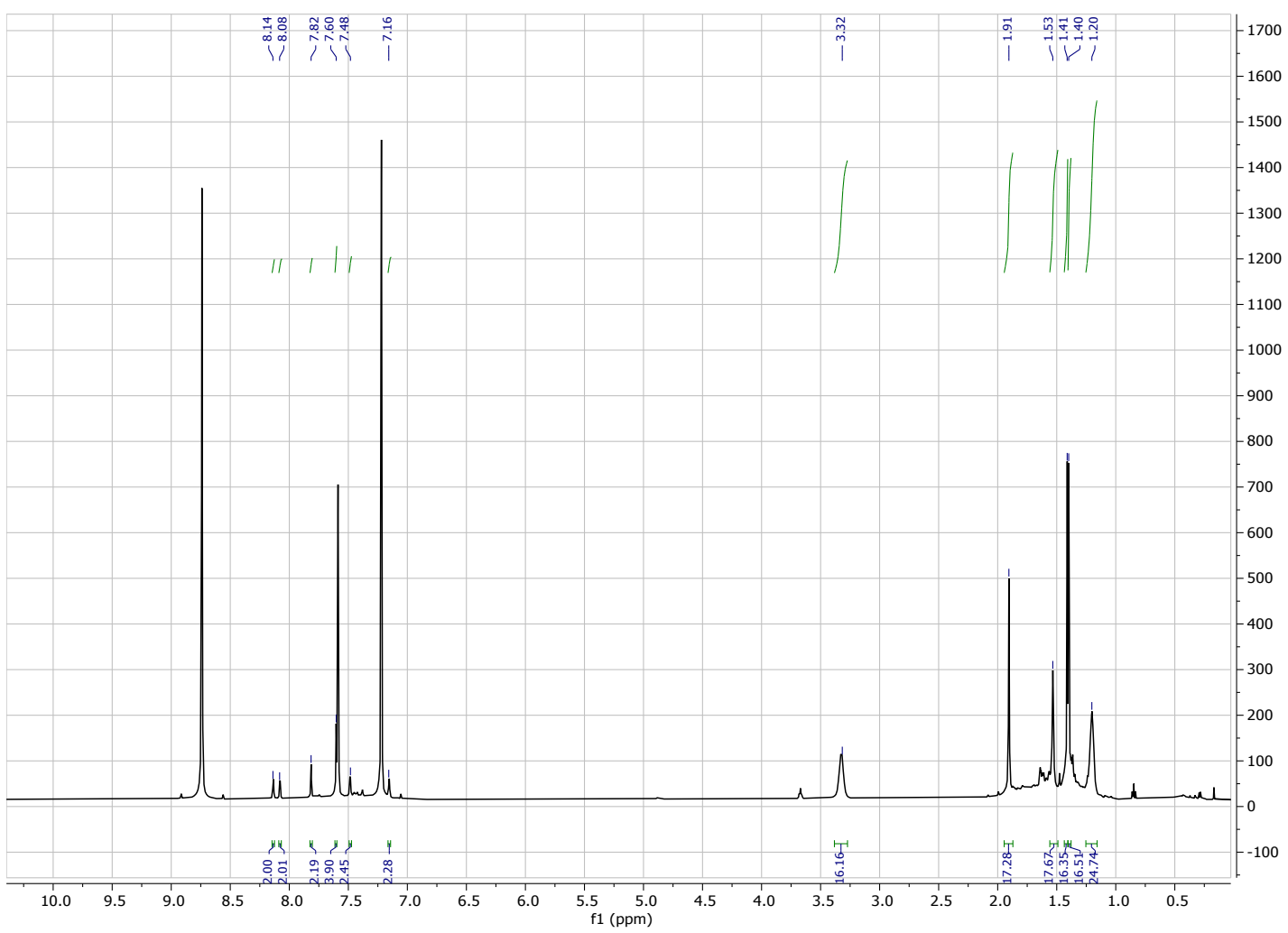

Figure S6: ${ }^{13} \mathrm{C}\left\{{ }^{1} \mathrm{H}\right\}$ NMR spectrum of $\left[\mathrm{NEt}_{4}\right]_{2}\left[\mathrm{Ce}_{2} \mathrm{Cl}{ }_{6}\left(p T P^{t}\right)\right]$ in $\mathrm{C}_{5} \mathrm{D}_{5} \mathrm{~N}$. 


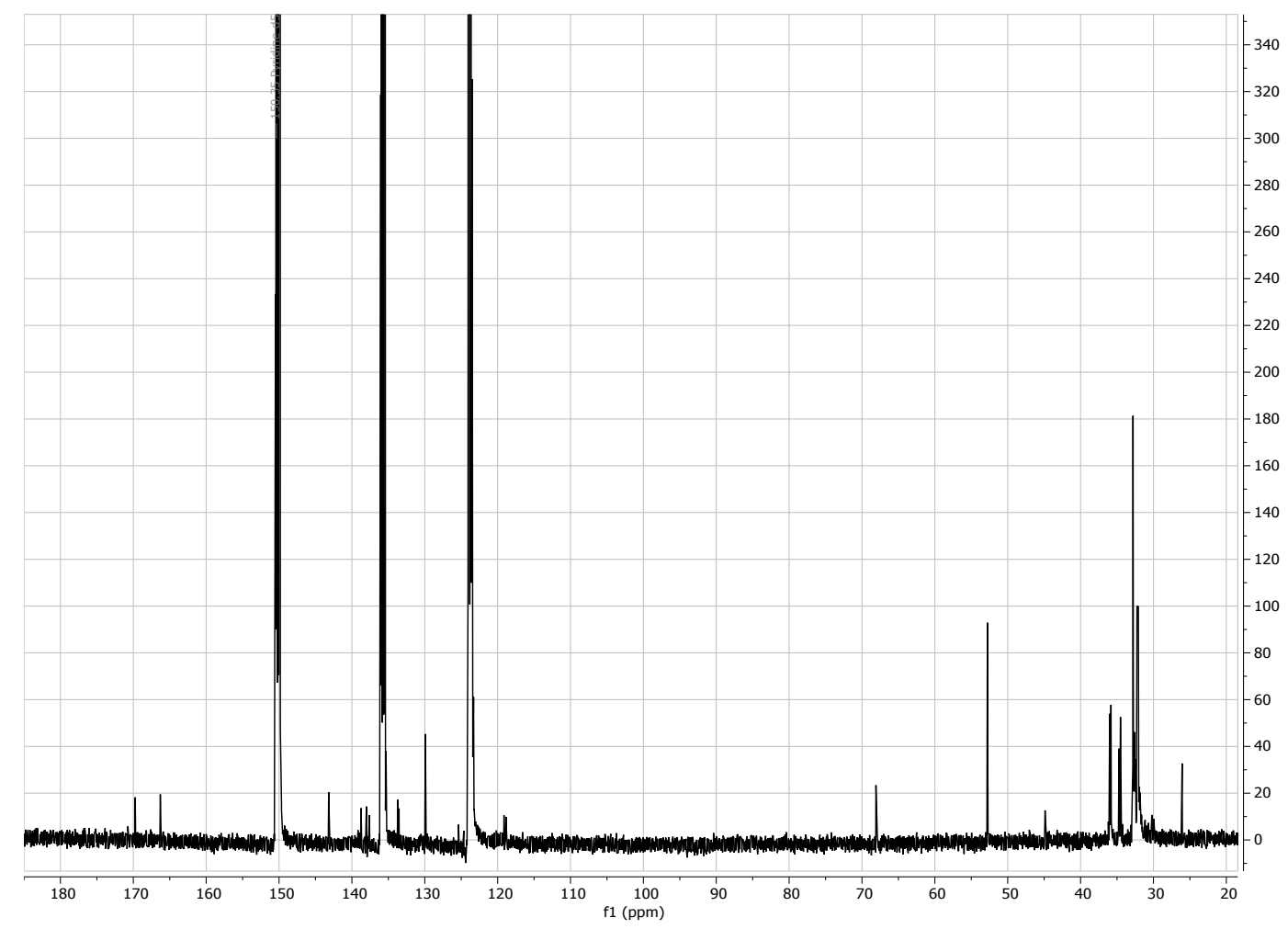

$\mathrm{S2.4}\left[\mathrm{NEt}_{4}\right]_{2}\left[\mathrm{Ce}_{2} \mathrm{Cl}_{2}\left(\mathrm{OSiMe}_{3}\right)_{4}\left(p \mathrm{TPt}^{\mathrm{t}}\right)\right]$

\section{Method A}

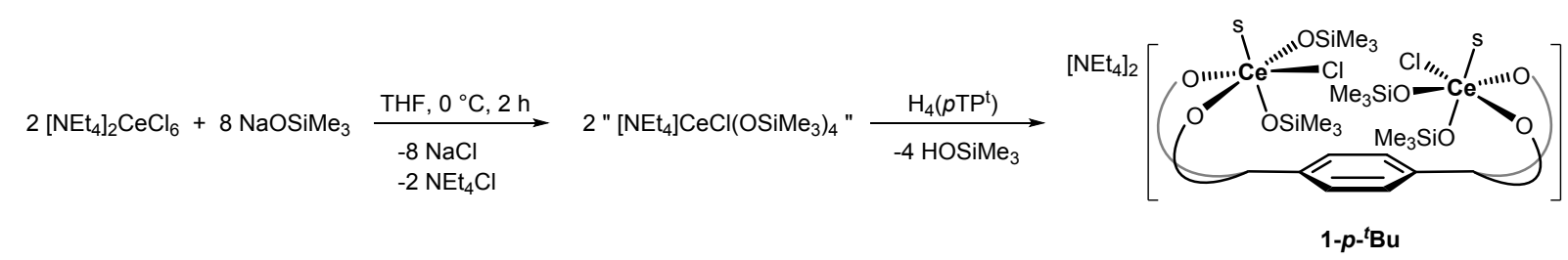

Equation S4: Synthesis of $\left[\mathrm{NEt}_{4}\right]_{2}\left[\mathrm{Ce}_{2} \mathrm{Cl}_{2}(\mathrm{OSiMe})_{2}\left(p \mathrm{TP}^{\mathrm{t}}\right)\right]$ by in situ formation of a "Ce-siloxide" complex.

[ $\left.\mathrm{NEt}_{4}\right]_{2} \mathrm{CeCl}_{6}$ (613 mg, $1 \mathrm{mmol}, 2$ equiv.) and sodium trimethylsiloxide ( $449 \mathrm{mg}, 4 \mathrm{mmol}, 8$ equiv.) were combined in a Schlenk flask, cooled in a salt-ice bath, and suspended in THF (15 mL). The yellow suspension was stirred at $0{ }^{\circ} \mathrm{C}$ for 2 hours before adding dropwise to a THF solution $(15 \mathrm{~mL})$ of $\mathrm{H}_{4}\left(p \mathrm{TP}^{\mathrm{t}}\right)(462 \mathrm{mg}, 0.5 \mathrm{mmol}, 1$ equiv.) over a period of 20 minutes. An initial green colour was observed which afterwards turned red-brown once the addition was completed. The reaction was stirred for a further 16 hours and the now purple mixture was centrifuged, filtered via cannula and the solids extracted with toluene $(2 \times 10 \mathrm{~mL})$. The filtrates were combined, volatiles were removed under reduced pressure giving the crude product, which was recrystallised from hot toluene yielding $\left[\mathrm{NEt}_{4}\right]_{2}\left[\mathrm{Ce}_{2} \mathrm{Cl}_{2}\left(\mathrm{OSiMe}_{3}\right)_{4}\left(p \mathrm{TP}^{\mathrm{t}}\right)\right]$ 1- $\boldsymbol{p}$-t'Bu as a purple solid (740 $\left.\mathrm{mg}, 72 \%\right)$.

${ }^{1} \mathrm{H}$ NMR $\left(500 \mathrm{MHz}\right.$, pyridine- $\left.\mathrm{d}_{5}\right) \delta_{\mathrm{H}} 7.64(\mathrm{~s}, 4 \mathrm{H}), 7.63(\mathrm{~d}, \mathrm{~J}=2.4 \mathrm{~Hz}, 4 \mathrm{H}), 7.34(\mathrm{~d}, \mathrm{~J}=2.4 \mathrm{~Hz}, 4 \mathrm{H}), 6.96(\mathrm{~s}, 1 \mathrm{H}$, benzylic $\mathrm{C}-\mathrm{H}), 6.88(\mathrm{~s}, 1 \mathrm{H}$, benzylic $\mathrm{C}-\mathrm{H}), 3.21\left(\mathrm{q}, 16 \mathrm{H},\left({ }^{+} \mathrm{N}\left(\mathrm{C}_{2} \mathrm{CH}_{3}\right)_{4}\right), 1.94(\mathrm{~s}, 36 \mathrm{H}, \mathrm{tBu}), 1.40(\mathrm{~s}, 36 \mathrm{H}, \mathrm{tBu}), 1.17(\mathrm{t}, 24 \mathrm{H}\right.$, $\left({ }^{+} \mathrm{N}\left(\mathrm{CH}_{2} \mathrm{CH}_{3}\right)_{4}\right), 0.43\left(\mathrm{~s}, 36 \mathrm{H}, \mathrm{OSiMe}_{3}\right)$.

${ }^{13} \mathrm{C}$ DEPTQ NMR (126 MHz, pyridine- $d_{5}$ ) $\delta_{\mathrm{C}} 167.4$ (q, ArC-O), 144.8 (q, ArC), 138.5 (q, ArC), $138.4(q, \operatorname{ArC}), 135.4$ (q, ArC), 129.2 (spacer, ArC-H), $125.2(\operatorname{ArC}-H), 119.9(\operatorname{ArC}-H), 53.2\left({ }^{+} \mathrm{N}\left(\mathrm{CH}_{2} \mathrm{CH}_{3}\right)_{4}\right), 36.5\left(\mathrm{q},{ }^{\mathrm{t}} \mathrm{Bu}\right), 35.0\left(\mathrm{q},{ }^{\mathrm{t}} \mathrm{Bu}\right), 33.2$ $\left(\mathrm{CH}_{3},{ }^{\mathrm{t}} \mathrm{Bu}\right), 32.7\left(\mathrm{CH}_{3},{ }^{\mathrm{t}} \mathrm{Bu}\right), 8.0\left({ }^{+} \mathrm{N}\left(\mathrm{CH}_{2} \mathrm{CH}_{3}\right)_{4}\right), 5.4\left(\mathrm{CH}_{3}, \mathrm{OSiMe}_{3}\right)$. 
${ }^{29} \mathrm{Si} N \mathrm{NMR}\left(99 \mathrm{MHz}\right.$, pyridine- $\left.\mathrm{d}_{5}\right) \delta_{\mathrm{Si}} 7.47$

Elemental analysis $\left(\left[\mathrm{NEt}_{4}\right]_{2}\left[\mathrm{Ce}_{2} \mathrm{Cl}_{2}\left(\mathrm{OSiMe}_{3}\right)_{4}\left(p \mathrm{TPt}^{\mathrm{t}}\right)(\mathrm{THF})_{2}\right]\right): \mathrm{C} 59.11 \%, \mathrm{H} 8.83 \%, \mathrm{~N} 1.38 \%$ calculated; $\mathrm{C} 58.73 \%, \mathrm{H}$ $8.81 \%$, N $1.41 \%$ found.

IR: $v_{\max } / \mathrm{cm}^{-1} 2952 \mathrm{w}, 1434 \mathrm{w}, 1251 \mathrm{~m}, 1239 \mathrm{~m}, 981 \mathrm{w}, 897 \mathrm{~s}, 832 \mathrm{~s}, 744 \mathrm{~m}$.

\section{Method B}
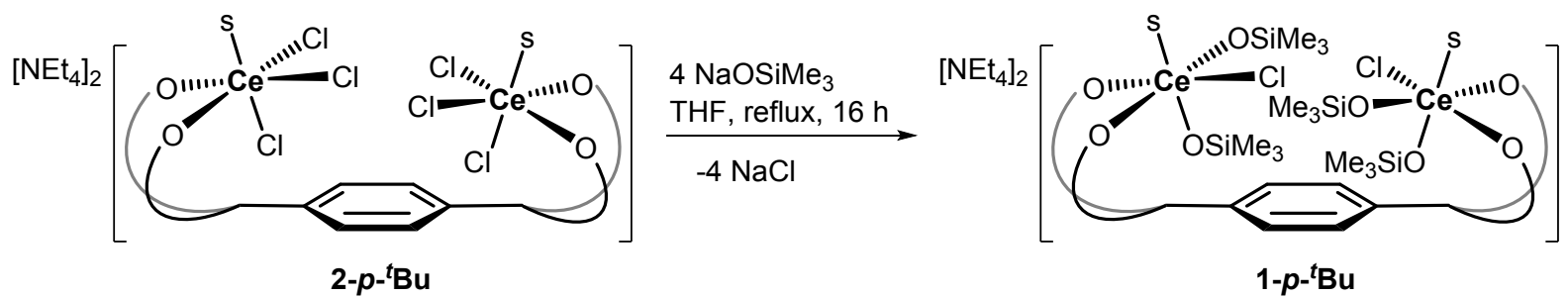

Equation S5: Synthesis of $\left[\mathrm{NEt}_{4}\right]_{2}\left[\mathrm{Ce}_{2} \mathrm{Cl}_{2}\left(\mathrm{OSiMe}_{3}\right)_{2}\left(p \mathrm{pP}^{\mathrm{t}}\right)\right]$ from $2-\boldsymbol{p}-{ }^{\mathrm{t}} \mathrm{Bu}$.

To a purple THF solution $(20 \mathrm{~mL})$ of $\left[\mathrm{NEt}_{4}\right]_{2}\left[\mathrm{Ce}_{2} \mathrm{Cl}_{6}\left(p T \mathrm{P}^{\mathrm{t}}\right)\right](1.00 \mathrm{~g}, 0.61 \mathrm{mmol}, 1$ equiv.) was added, with stirring, a clear THF solution $(20 \mathrm{~mL}$ ) of sodium trimethylsiloxide $(273 \mathrm{mg}, 2.44 \mathrm{mmol}, 4$ equiv.). The resulting purple solution was heated under reflux for 16 hours before allowing to cool to room temperature, centrifuged, filtered via cannula and the solids extracted with toluene $(2 \times 10 \mathrm{~mL})$. The filtrates were combined, volatiles were removed under reduced pressure giving the crude product, which was recrystallised from hot toluene yielding $\left[\mathrm{NEt}_{4}\right]_{2}\left[\mathrm{Ce}_{2} \mathrm{Cl}_{2}\left(\mathrm{OSiMe}_{3}\right)_{4}(\right.$ TTPt $\left.)\right]$ 1-p-tBu as a purple solid (790 mg, 69\%).

Figure S7: ${ }^{1} \mathrm{H}$ NMR spectrum of $\left[\mathrm{NEt}_{4}\right]_{2}\left[\mathrm{Ce}_{2} \mathrm{Cl}_{2}\left(\mathrm{OSiMe}_{3}\right)_{4}\left(p T \mathrm{P}^{\mathrm{t}}\right)\right]$ in $\mathrm{C}_{5} \mathrm{D}_{5} \mathrm{~N}$.

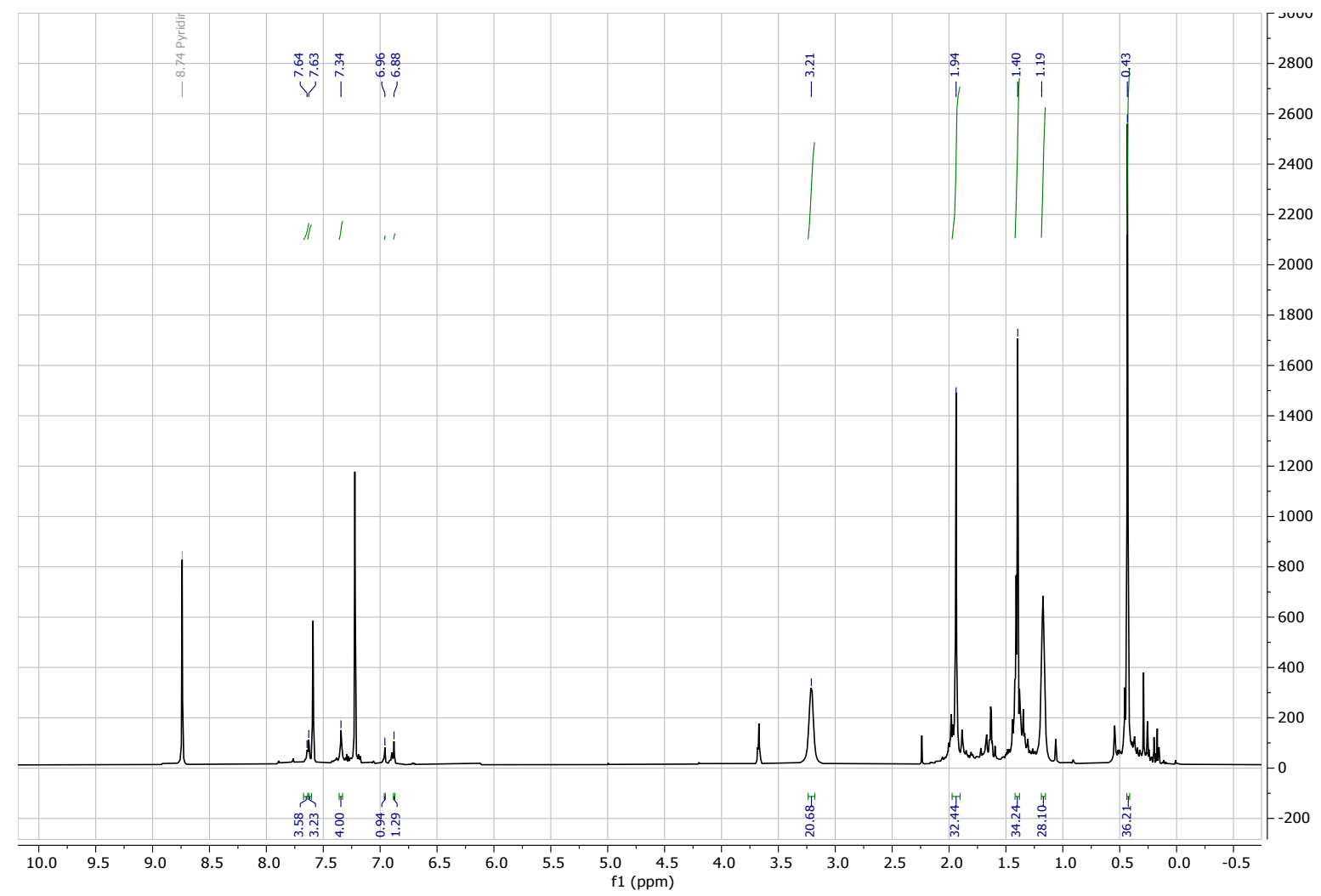


Figure S8: Section of variable temperature ${ }^{1} \mathrm{H}$ NMR spectra of $\left[\mathrm{NEt}_{4}\right]_{2}\left[\mathrm{Ce}_{2} \mathrm{Cl}_{2}\left(\mathrm{OSiMe}_{3}\right)_{4}\left(p T P^{t}\right)\right]$ in $\mathrm{C}_{5} \mathrm{D}_{5} \mathrm{~N}$.

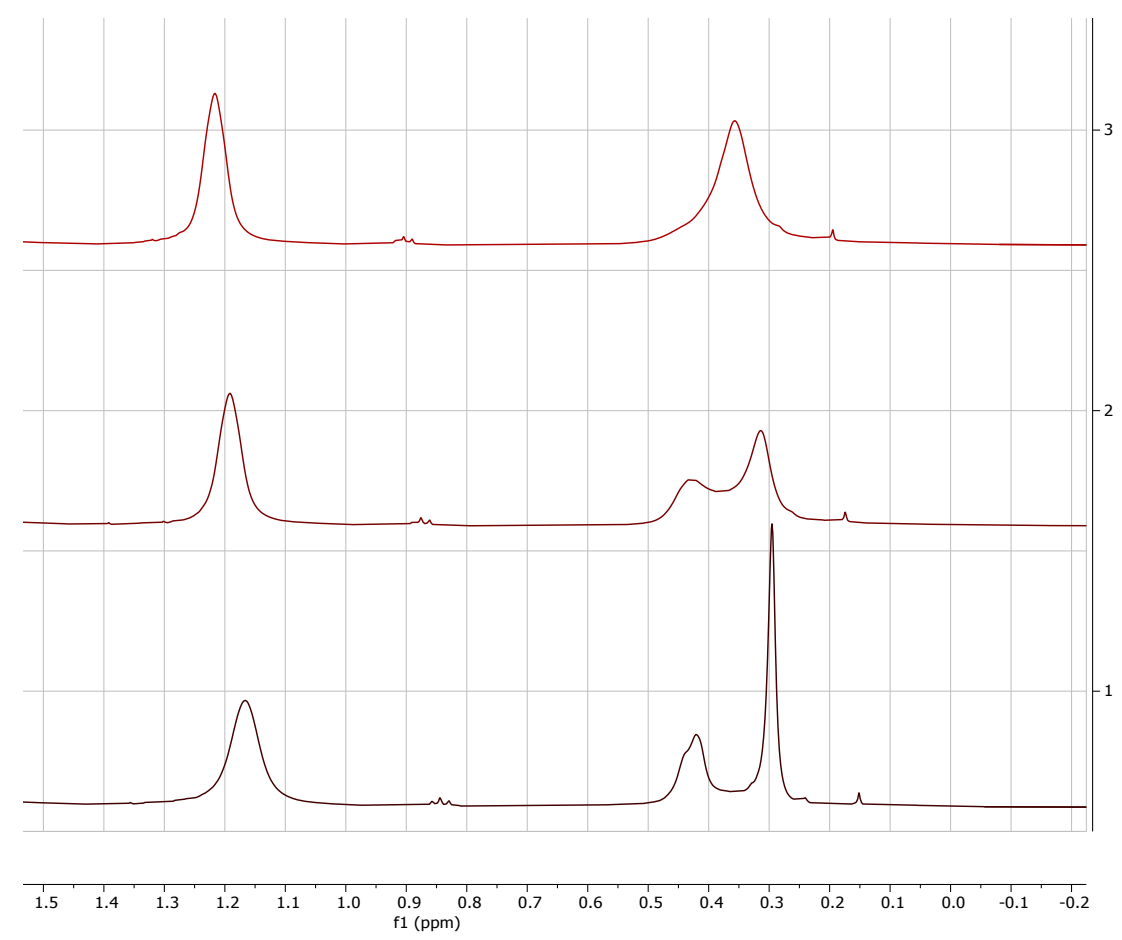

The top spectrum is at $340 \mathrm{~K}$ and shows a coalescence of both $\mathrm{OSiMe}_{3}$ signals into one, indicating that upon even mild heating, there is sufficient energy for free rotation between the two conformers.

The middle spectrum is at $320 \mathrm{~K}$.

The bottom spectrum is at $300 \mathrm{~K}$ and shows two signals for the $\mathrm{OSiMe}_{3}$ ligands, at 0.4 and 0.3 ppm - one each for the anti and syn conformers.

${ }^{1} \mathrm{H}-{ }^{1} \mathrm{H}$ NOESY spectrum of $\left[\mathrm{NEt}_{4}\right]_{2}\left[\mathrm{Ce}_{2} \mathrm{Cl}_{2}\left(\mathrm{OSiMe}_{3}\right)_{4}\left(p T \mathrm{PPt}^{\mathrm{t}}\right)\right]$ in $\mathrm{C}_{5} \mathrm{D}_{5} \mathrm{~N}$. No cross-peaks are observable that correspond to siloxide ligands in close proximity.

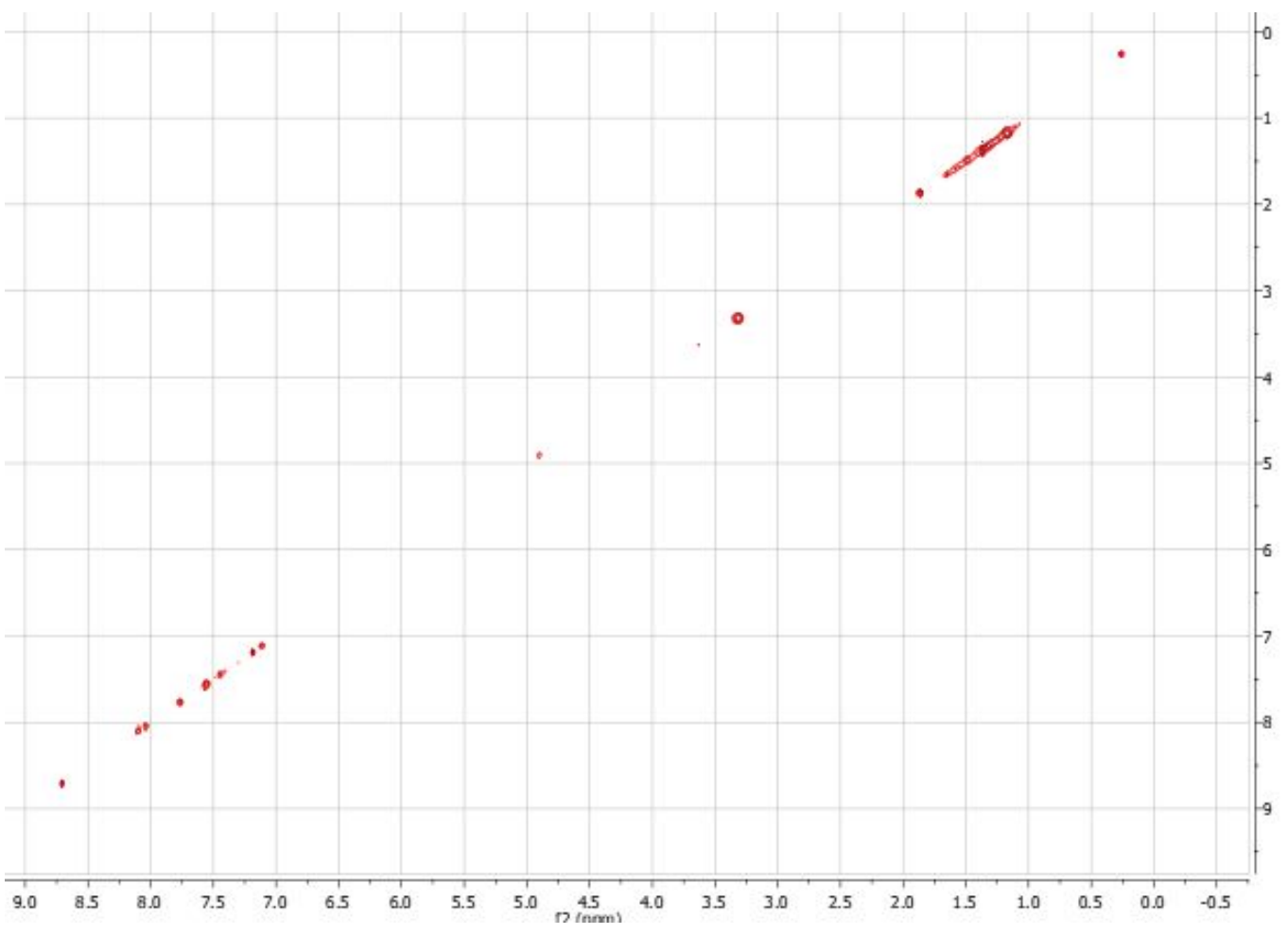


Figure S9: ${ }^{13} \mathrm{C}$ DEPTQ NMR spectrum of $\left[\mathrm{NEt}_{4}\right]_{2}\left[\mathrm{Ce}_{2} \mathrm{Cl}_{2}\left(\mathrm{OSiMe}_{3}\right)_{4}\left(p T \mathrm{Pt}^{\mathrm{t}}\right)\right]$ in $\mathrm{C}_{5} \mathrm{D}_{5} \mathrm{~N}$.

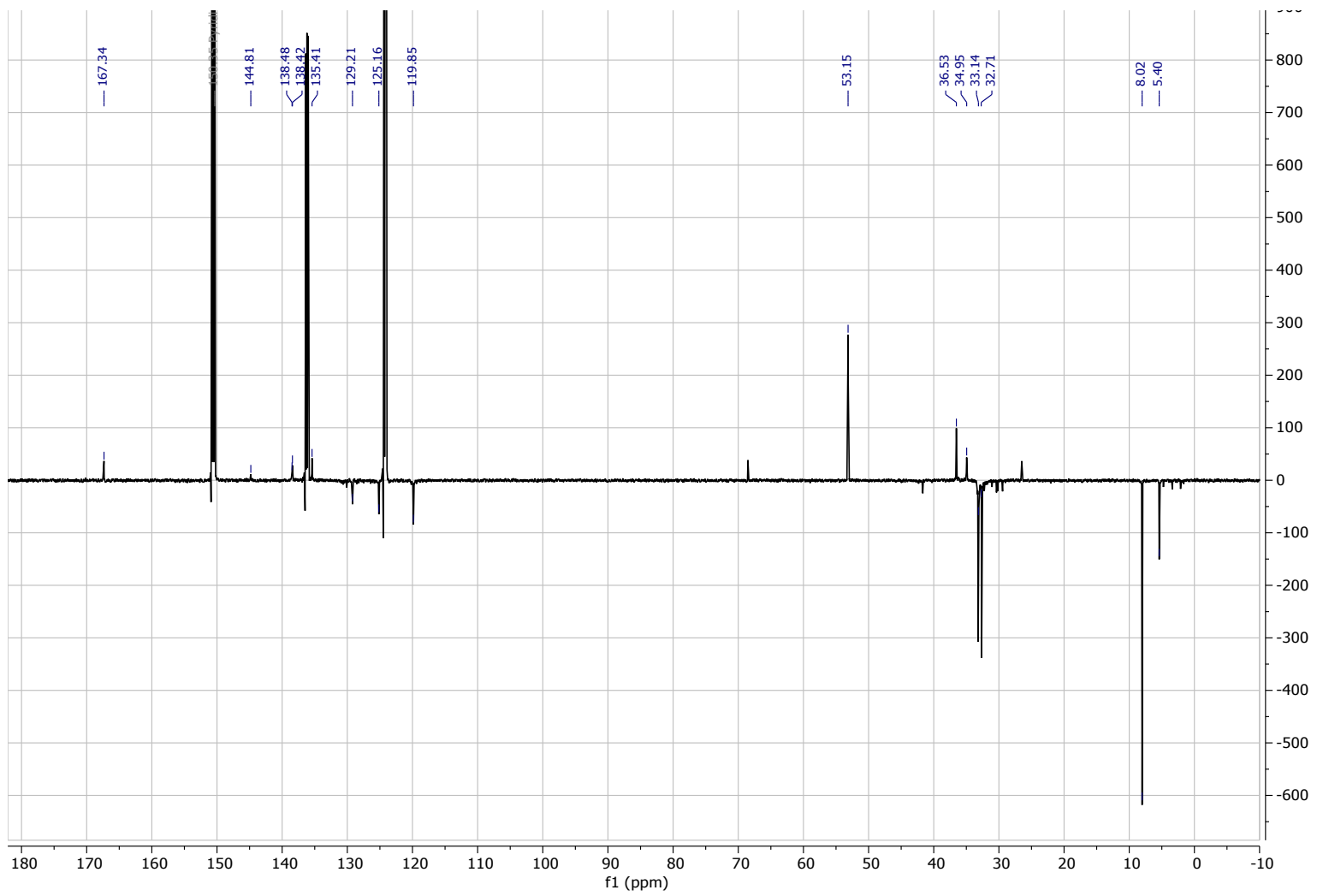

$\mathrm{S2.5}\left[\mathrm{NEt}_{4}\right]_{2}\left[\mathrm{Ce}_{2} \mathrm{Cl}_{2}\left(\mathrm{OSiMe}_{3}\right)_{4}\left(m \mathrm{MPt}^{\mathrm{t}}\right)\right]$

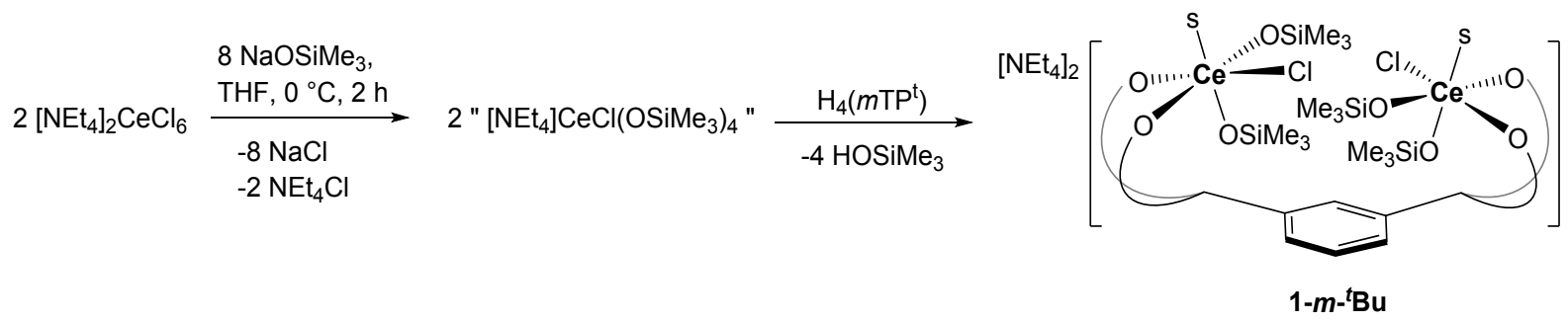

Equation S6: Synthesis of $\left[\mathrm{NEt}_{4}\right]_{2}\left[\mathrm{Ce}_{2} \mathrm{Cl}_{2}(\mathrm{OSiMe})_{2}\left(m T \mathrm{P}^{\mathrm{t}}\right)\right]$.

By analogous procedure to S2.4A.

$\left[\mathrm{NEt}_{4}\right]_{2} \mathrm{CeCl}_{6}(681 \mathrm{mg}, 1.11 \mathrm{mmol}, 2$ equiv.) and sodium trimethylsiloxide (498 mg, $4.44 \mathrm{mmol}, 8$ equiv.) were combined in a Schlenk flask, cooled in a salt-ice bath, and suspended in THF (15 mL). The yellow suspension was stirred at $0{ }^{\circ} \mathrm{C}$ for 2 hours before adding dropwise to a THF solution (15 mL) of $\mathrm{H}_{4}(\mathrm{mTPt})$ (513 mg, $0.55 \mathrm{mmol}, 1$ equiv.). An initial green colour was observed which afterwards turned red-brown once the addition was completed. The reaction was stirred for a further 16 hours and worked up in an identical manner to S2.4A. The purple solid $\left[\mathrm{NEt}_{4}\right]_{2}\left[\mathrm{Ce}_{2} \mathrm{Cl}_{2}\left(\mathrm{OSiMe}_{3}\right)_{4}\left(m \mathrm{mP}^{\mathrm{t}}\right)\right]$ 1- $m$-' ${ }^{\mathrm{B} u}$ was obtained (735 mg, $\left.71 \%\right)$.

${ }^{1} \mathrm{H}$ NMR $\left(500 \mathrm{MHz}\right.$, pyridine- $\left.\mathrm{d}_{5}\right) \delta_{\mathrm{H}} 7.56(\mathrm{~m}, 1 \mathrm{H}), 7.53(\mathrm{~m}, 1 \mathrm{H}), 7.43(\mathrm{~m}, 1 \mathrm{H}), 7.40(\mathrm{~m}, 1 \mathrm{H}) 7.35(\mathrm{~m}, 4 \mathrm{H}), 7.31(\mathrm{~m}$, $4 \mathrm{H}), 6.94(\mathrm{~s}, 2 \mathrm{H}$, benzylic $\mathrm{C}-\mathrm{H}) 3.33\left(\mathrm{q}, 16 \mathrm{H},\left({ }^{+} \mathrm{N}\left(\mathrm{CH}_{2} \mathrm{CH}_{3}\right)_{4}\right), 2.02\left(\mathrm{br} \mathrm{s}, 36 \mathrm{H},{ }^{\mathrm{t}} \mathrm{Bu}\right), 1.36\left(\mathrm{~s}, 36 \mathrm{H},{ }^{\mathrm{t}} \mathrm{Bu}\right), 1.23(\mathrm{t}, 24 \mathrm{H}\right.$, $\left({ }^{+} \mathrm{N}\left(\mathrm{CH}_{2} \mathrm{CH}_{3}\right)_{4}\right), 0.45$ (br s, 36H, OSiMe $)_{3}$.

${ }^{13} \mathrm{C}$ DEPTQ NMR (126 MHz, pyridine- $d_{5}$ ) $\delta_{\mathrm{C}} 166.6$ (q, ArC-O), 147.0 (q, ArC), 146.6 (q, ArC), 134.9 (q, ArC), 134.8 (q, ArC), $119.7(\mathrm{ArC}-\mathrm{H}), 119.5(\mathrm{ArC}-\mathrm{H}), 52.8\left({ }^{+} \mathrm{N}\left(\mathrm{CH}_{2} \mathrm{CH}_{3}\right)_{4}\right), 36.2\left(\mathrm{q},{ }^{\mathrm{t} B u}\right), 36.0\left(\mathrm{q},{ }^{\mathrm{t} B u}\right), 33.0\left(\mathrm{CH}_{3},{ }^{\mathrm{t} B u}\right), 32.9\left(\mathrm{CH}_{3}\right.$, $\left.{ }^{t} \mathrm{Bu}\right), 32.4\left(\mathrm{CH}_{3},{ }^{\mathrm{t} B u}\right), 31.9\left(\mathrm{CH}_{3},{ }^{\mathrm{t}} \mathrm{Bu}\right), 7.6\left({ }^{+} \mathrm{N}\left(\mathrm{CH}_{2} \mathrm{CH}_{3}\right)_{4}\right), 3.0\left(\mathrm{CH}_{3}, \mathrm{OSiMe}_{3}\right)$. 
${ }^{29} \mathrm{Si}$ NMR (99 MHz, pyridine- $\left.\mathrm{d}_{5}\right) \delta_{\mathrm{si}} 1.40$.

Elemental analysis $\left(\left[\mathrm{NEt}_{4}\right]_{2}\left[\mathrm{Ce}_{2} \mathrm{Cl}_{2}\left(\mathrm{OSiMe}_{3}\right)_{4}(m \mathrm{TPt})(\mathrm{THF})_{2}\right]\right): \mathrm{C} 59.11 \%, \mathrm{H} 8.83 \%, \mathrm{~N} 1.38 \%$ calculated; $\mathrm{C} 59.58 \%, \mathrm{H}$ $8.62 \%, N 1.53 \%$ found.

IR: $v_{\max } / \mathrm{cm}^{-1} 2952 \mathrm{w}, 1595 \mathrm{w}, 1435 \mathrm{w}, 1255 \mathrm{~m}, 972 \mathrm{w}, 891 \mathrm{~m}, 832 \mathrm{~s}, 744 \mathrm{~m}$.

Figure S10: ${ }^{1} \mathrm{H}$ NMR spectrum of $\left[\mathrm{NEt}_{4}\right]_{2}\left[\mathrm{Ce}_{2} \mathrm{Cl}_{2}\left(\mathrm{OSiMe}_{3}\right)_{4}\left(m T \mathrm{Pt}^{\mathrm{t}}\right)\right]$ in $\mathrm{C}_{5} \mathrm{D}_{5} \mathrm{~N}$.

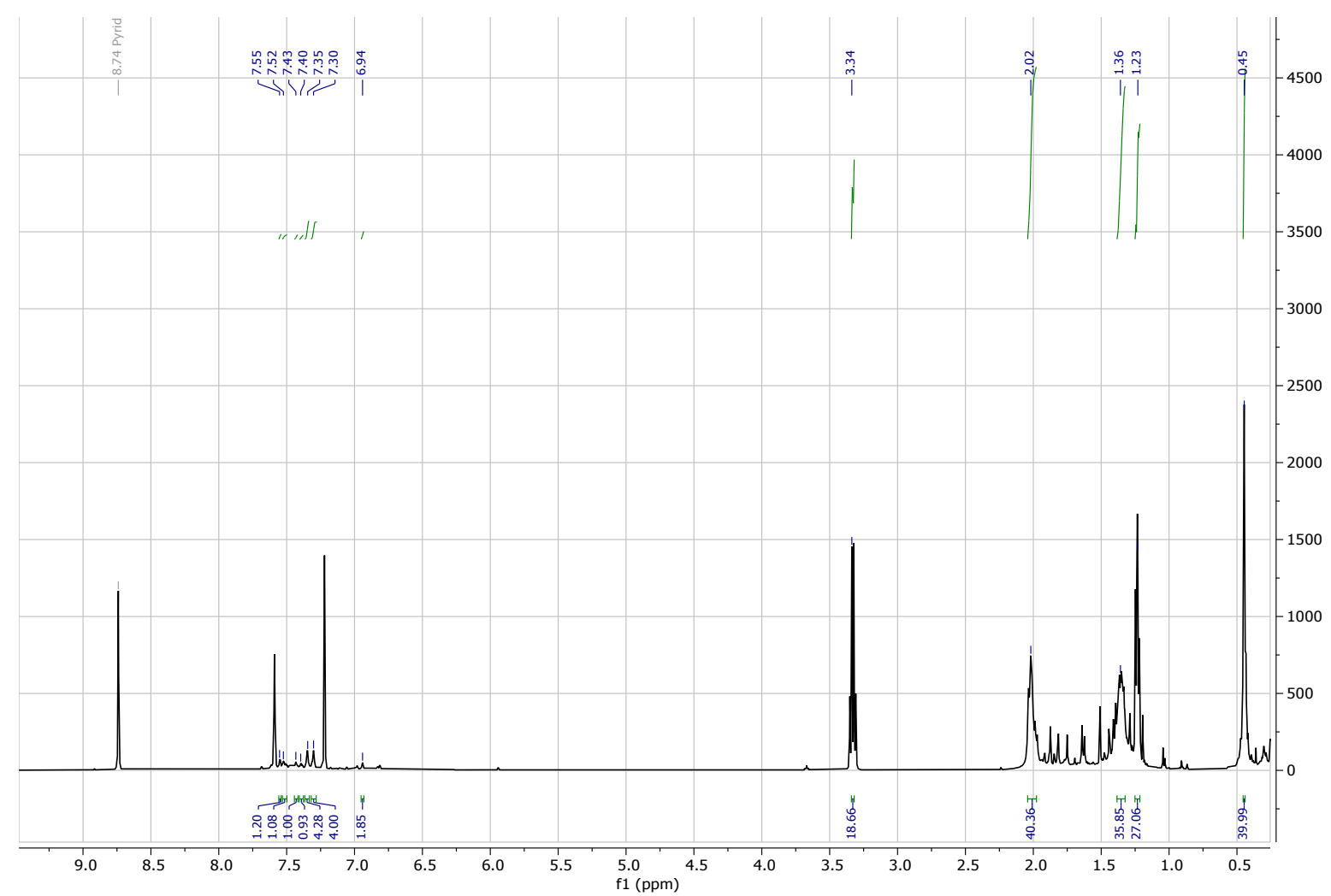


Figure S11: ${ }^{13} \mathrm{C}$ DEPTQ NMR spectrum of $\left[\mathrm{NEt}_{4}\right]_{2}\left[\mathrm{Ce}_{2} \mathrm{Cl}_{2}\left(\mathrm{OSiMe}_{3}\right)_{4}\left(m T P^{t}\right)\right]$ in $\mathrm{C}_{5} \mathrm{D}_{5} \mathrm{~N}$.

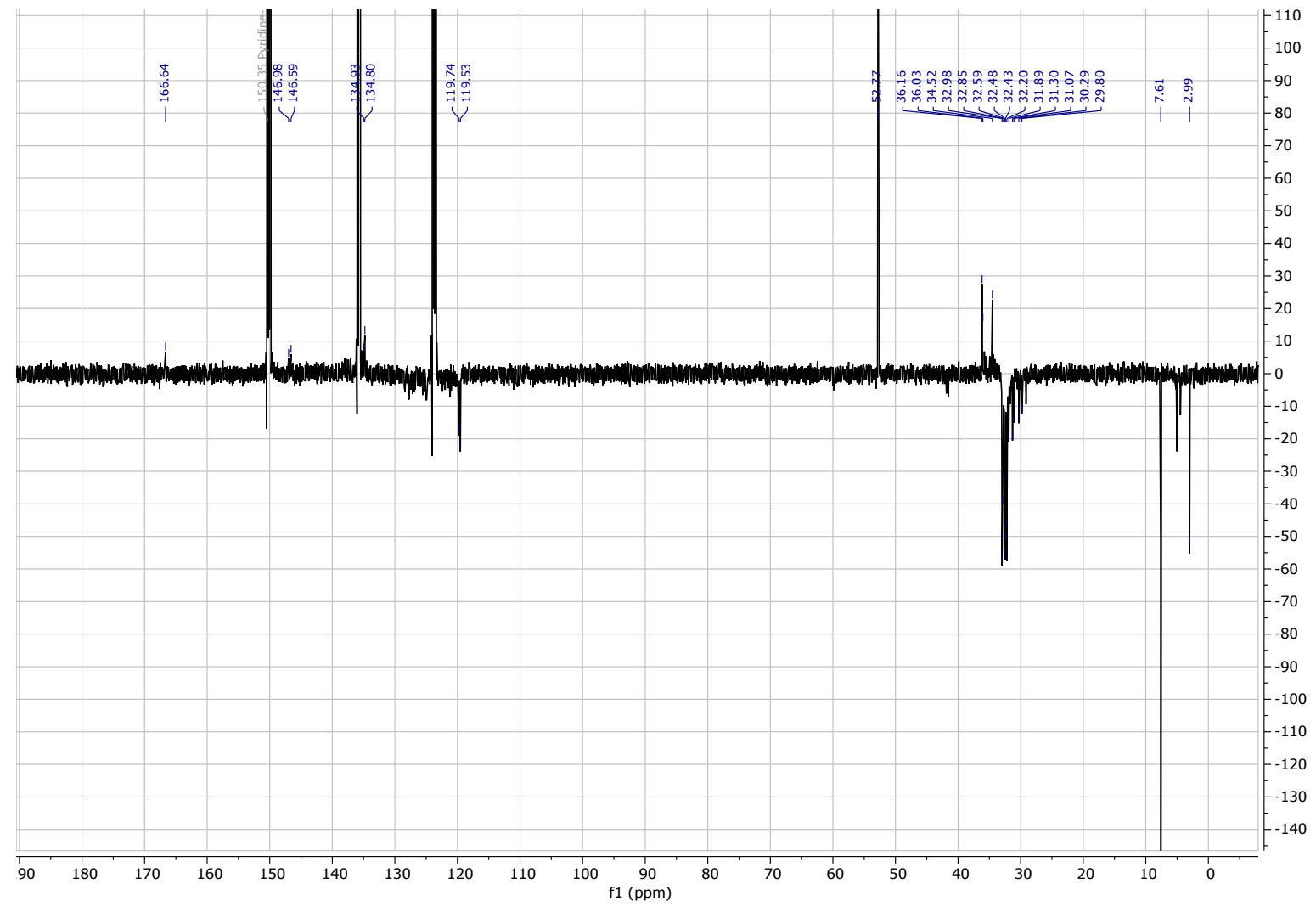

\section{$\mathrm{S2.6}\left[\mathrm{NEt}_{4}\right]_{2}\left[\mathrm{Ce}_{2} \mathrm{Cl}_{2}\left(\mathrm{OSiMe}_{3}\right)_{4}\left(p \mathrm{TP}^{\mathrm{Ad}}\right)\right]$}

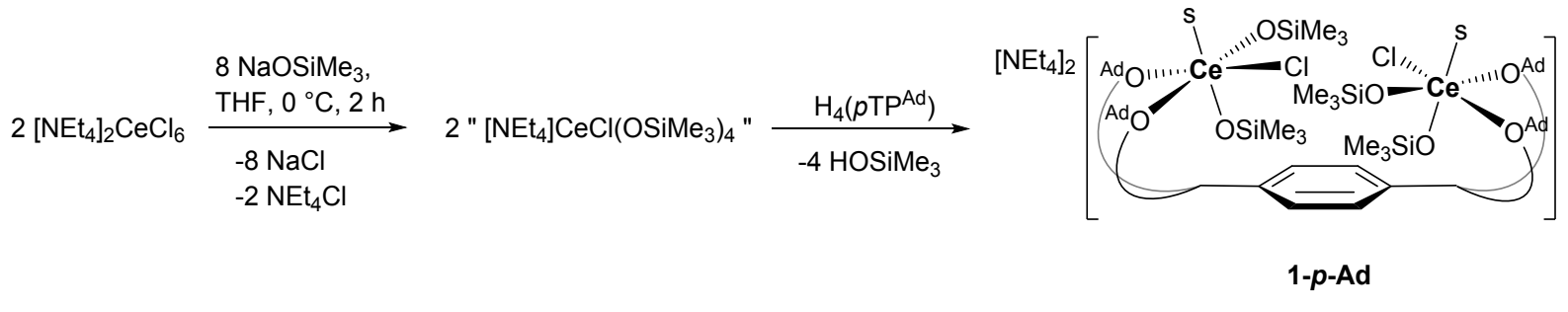

Equation S7: Synthesis of $\left[\mathrm{NEt}_{4}\right]_{2}\left[\mathrm{Ce}_{2} \mathrm{Cl}_{2}\left(\mathrm{OSiMe}_{3}\right)_{2}\left(p \mathrm{TPAd}^{\mathrm{Ad}}\right)\right]$.

By analogous procedure to S2.4A.

$\left[\mathrm{NEt}_{4}\right]_{2} \mathrm{CeCl}_{6}$ (515 mg, $0.840 \mathrm{mmol}, 2$ equiv.) and sodium trimethylsiloxide ( $377 \mathrm{mg}, 3.36 \mathrm{mmol}, 8$ equiv.) were combined in a Schlenk flask, cooled in a salt-ice bath, and suspended in THF (15 mL). The yellow suspension was stirred at $0{ }^{\circ} \mathrm{C}$ for 2 hours before adding dropwise to a THF solution ( $15 \mathrm{~mL}$ ) of $\mathrm{H}_{4}\left(p \mathrm{TP}^{\mathrm{Ad}}\right)(623 \mathrm{mg}, 0.42 \mathrm{mmol}, 1$ equiv.). An initial green colour was observed which afterwards turned red-brown once the addition was completed. The reaction was stirred for a further 16 hours and worked up in an identical manner to S2.4A. The purple solid $\left[\mathrm{NEt}_{4}\right]_{2}\left[\mathrm{Ce}_{2} \mathrm{Cl}_{2}\left(\mathrm{OSiMe}_{3}\right)_{4}\left(p \mathrm{TP}^{\mathrm{Ad}}\right)\right]$ 1-p-Ad was obtained (592 mg, 60\%).

${ }^{1} \mathrm{H}$ NMR $\left(500 \mathrm{MHz}\right.$, pyridine- $\left.\mathrm{d}_{5}\right) \delta_{\mathrm{H}} 7.21(\mathrm{~s}, 4 \mathrm{H}, \mathrm{Ar}-\mathrm{H}), 7.18-7.13(\mathrm{~m}, 4 \mathrm{H}, \mathrm{Ar}-\mathrm{H}), 7.02-6.96(\mathrm{~m}, 4 \mathrm{H}, \mathrm{Ar}-\mathrm{H}), 6.71(\mathrm{~s}$, $1 \mathrm{H}$, benzylic $\mathrm{C}-\mathrm{H}), 6.40(\mathrm{~s}, 1 \mathrm{H}$, benzylic $\mathrm{C}-\mathrm{H}), 3.02\left(\mathrm{q}, 16 \mathrm{H},\left({ }^{+} \mathrm{N}\left(\mathrm{CH}_{2} \mathrm{CH}_{3}\right)_{4}\right), 2.74-2.66\left(\mathrm{~m}, 18 \mathrm{H},-\mathrm{CH}_{2}\right), 2.44(\mathrm{~s}, 18 \mathrm{H})\right.$, $2.35(\mathrm{~s}, 6 \mathrm{H}), 2.28-2.20\left(\mathrm{~m}, 6 \mathrm{H},-\mathrm{CH}_{2}\right), 2.10(\mathrm{~s}, 6 \mathrm{H}), 1.96-1.76(\mathrm{~m}, 18 \mathrm{H}), 1.15\left(\mathrm{t}, 24 \mathrm{H},\left({ }^{+} \mathrm{N}\left(\mathrm{CH}_{2} \mathrm{CH}_{3}\right)_{4}\right), 0.40(\mathrm{~s}, 36 \mathrm{H}\right.$, $\left.\mathrm{OSiMe}_{3}\right)$. 
${ }^{13} \mathrm{C}$ DEPTQ NMR (126 MHz, pyridine- $d_{5}$ ) $\delta_{\mathrm{C}} 155.9$ (q, ArC-O), 138.6 (q, ArC), 137.2 (q, ArC), 129.9 (ArC-H), 129.1 (ArC-H), 128.3 (ArC-H), 127.9 (ArC-H), 126.2 (ArC-H) $53.0\left({ }^{+} \mathrm{N}\left(\mathrm{CH}_{2} \mathrm{CH}_{3}\right)_{4}\right), 42.2,41.5,41.3\left(-\mathrm{CH}_{2}\right), 38.5,38.3,37.90$ $\left(-\mathrm{CH}_{2}\right), 30.5,30.4,30.0\left(-\mathrm{CH}_{3}\right), 7.9\left({ }^{+} \mathrm{N}\left(\mathrm{CH}_{2} \mathrm{CH}_{3}\right)_{4}\right)$.

${ }^{29}$ Si NMR (99 MHz, pyridine- $\left.d_{5}\right) \delta_{\mathrm{Si}} 10.48$

Elemental analysis ([NEt $\left.]_{2}\left[\mathrm{Ce}_{2} \mathrm{Cl}_{2}\left(\mathrm{OSiMe}_{3}\right)_{4}(p \mathrm{TPAd})(\mathrm{THF})_{2}\right]\right): \mathrm{C} 61.82 \%, \mathrm{H} 8.25 \%, \mathrm{~N} 1.29 \%$ calculated; $\mathrm{C} 61.37 \%, \mathrm{H}$ $7.95 \%, N 1.28 \%$ found.

Figure S12: Section of variable temperature ${ }^{1} \mathrm{H}$ NMR spectra of $\left[\mathrm{NEt}_{4}\right]_{2}\left[\mathrm{Ce}_{2}\left(\mathrm{OSiMe}_{3}\right)_{6}\left(p \mathrm{TPd}^{\mathrm{Ad}}\right)\right]$ in $\mathrm{C}_{5} \mathrm{D}_{5} \mathrm{~N}$.

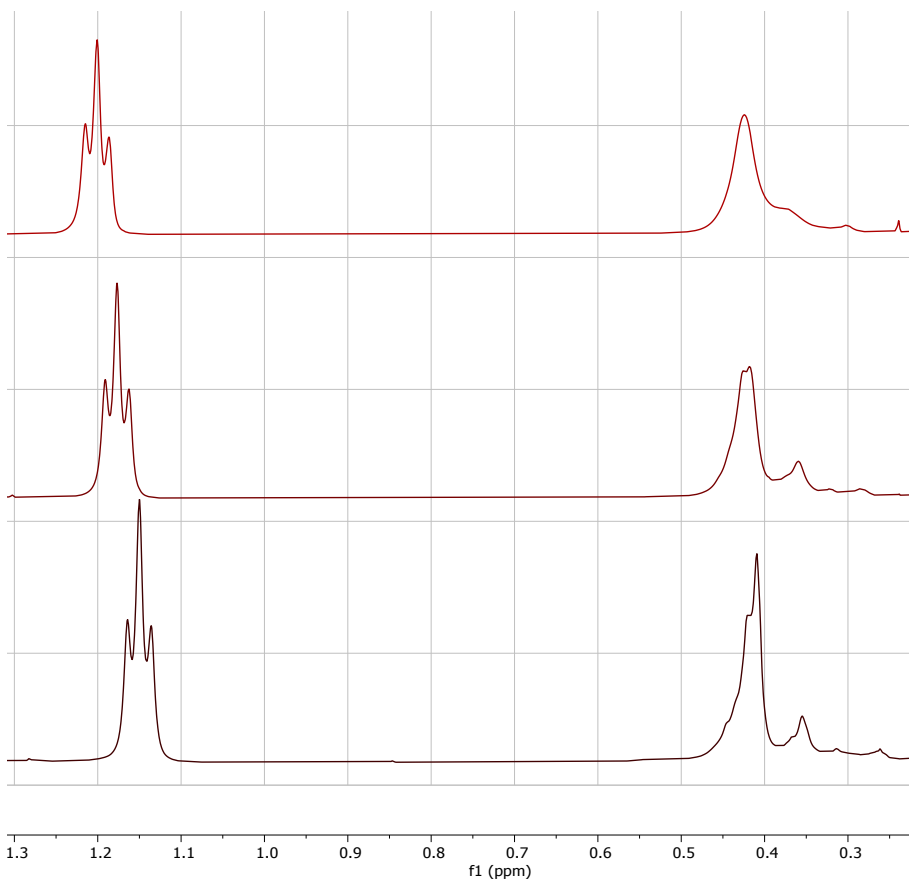

The top spectrum is at $340 \mathrm{~K}$ and shows a coalescence of both $\mathrm{OSiMe}_{3}$ signals into one, indicating that upon even mild heating, there is sufficient energy for free rotation between the two conformers.

The middle spectrum is at $320 \mathrm{~K}$.

The bottom spectrum is at $300 \mathrm{~K}$ and shows two signals for the $\mathrm{OSiMe}_{3}$ ligands, at 0.43 and 0.35 ppm - one each for the anti and syn conformers.

Figure S13: ${ }^{1} \mathrm{H}$ NMR spectrum of $\left[\mathrm{NEt}_{4}\right]_{2}\left[\mathrm{Ce}_{2} \mathrm{Cl}_{2}\left(\mathrm{OSiMe}_{3}\right)_{4}(p \mathrm{TPAd})\right]$ in $\mathrm{C}_{\llcorner}$

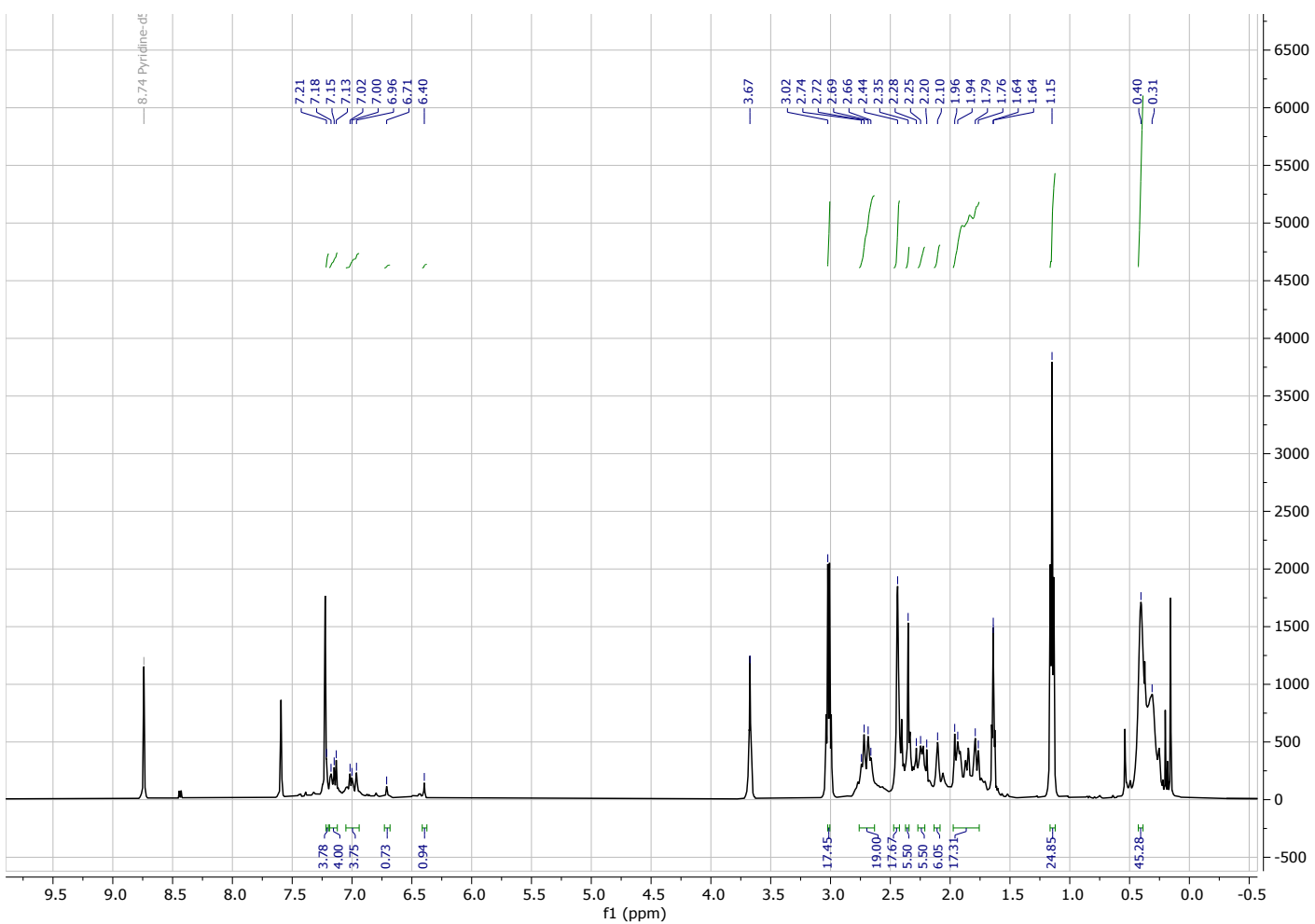


Figure S14: ${ }^{13} \mathrm{C}$ DEPTQ NMR spectrum of $\left[\mathrm{NEt}_{4}\right]_{2}\left[\mathrm{Ce}_{2} \mathrm{Cl}_{2}\left(\mathrm{OSiMe}_{3}\right)_{4}\left(p T P^{\mathrm{Ad}}\right)\right]$ in $\mathrm{C}_{5} \mathrm{D}_{5} \mathrm{~N}$.

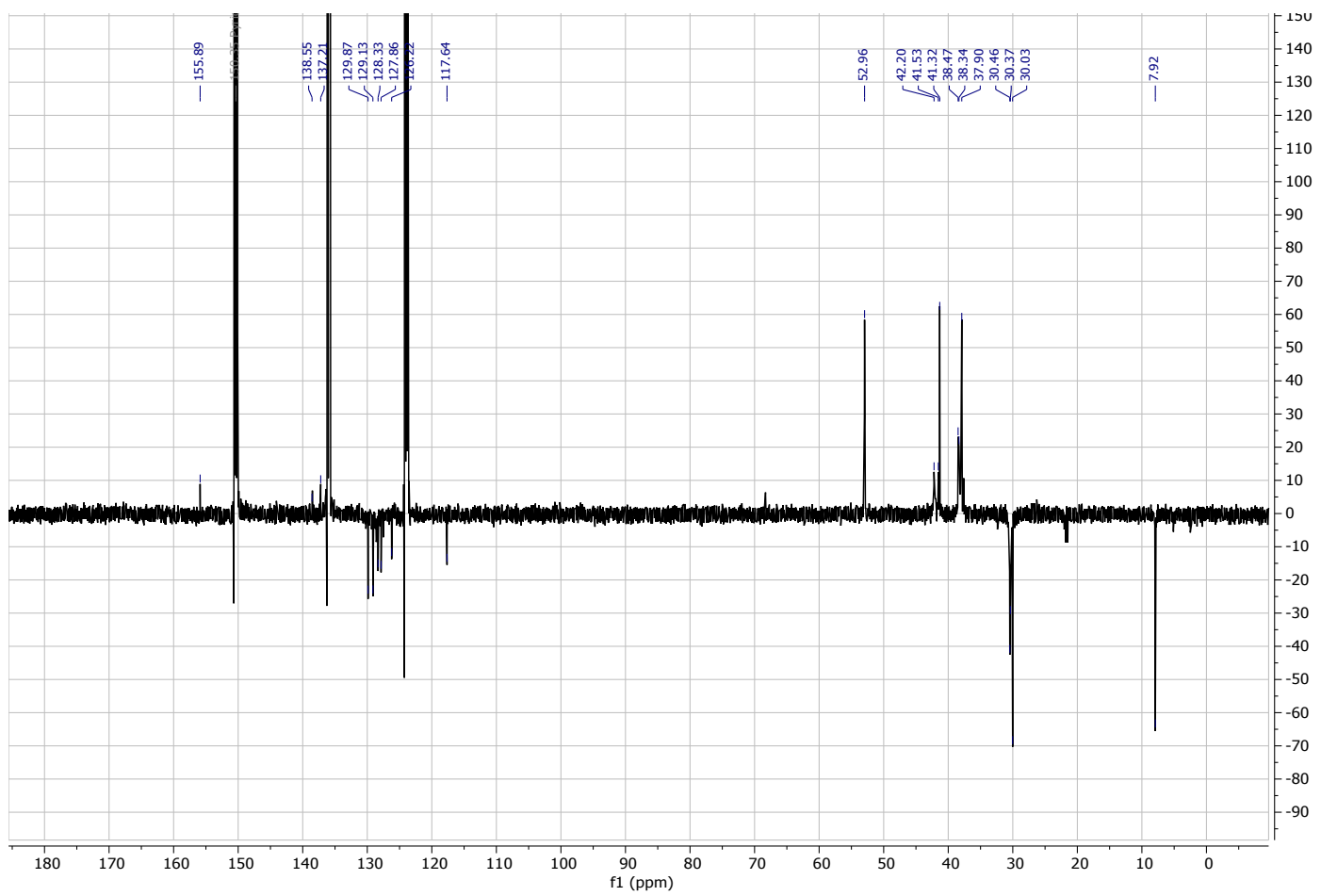

\section{$\mathrm{S2.7}\left[\mathrm{NEt}_{4}\right]_{2}\left[\mathrm{Ce}_{2} \mathrm{Cl}_{2}\left(\mathrm{OSiMe}_{3}\right)_{4}(m \mathrm{TPAd})\right]$}

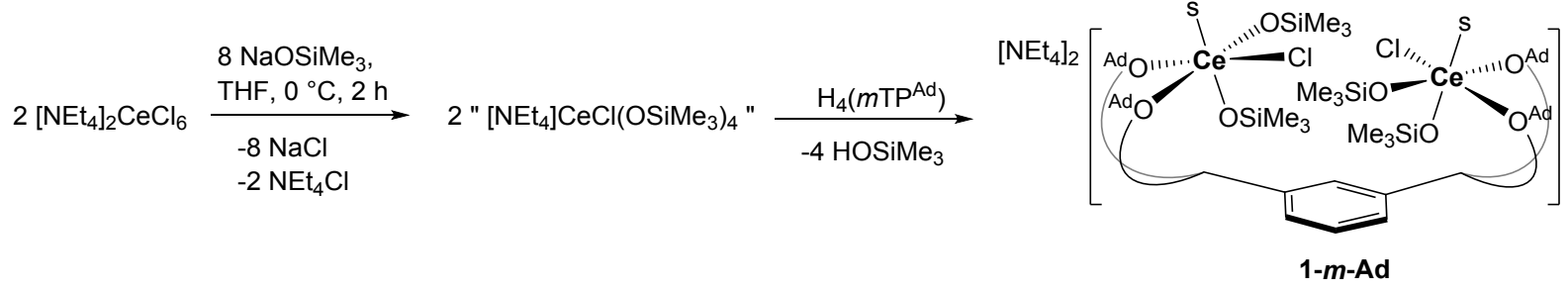

Equation S8: Synthesis of $\left[\mathrm{NEt}_{4}\right]_{2}\left[\mathrm{Ce}_{2} \mathrm{Cl}_{2}\left(\mathrm{OSiMe}_{3}\right)_{2}\left(m T \mathrm{PAd}^{\mathrm{Ad}}\right)\right]$.

By analogous procedure to S2.4A.

$\left[\mathrm{NEt}_{4}\right]_{2} \mathrm{CeCl}_{6}(515 \mathrm{mg}, 0.840 \mathrm{mmol}, 2$ equiv.) and sodium trimethylsiloxide $(377 \mathrm{mg}, 3.36 \mathrm{mmol}, 8$ equiv.) were combined in a Schlenk flask, cooled in a salt-ice bath, and suspended in THF ( $15 \mathrm{~mL})$. The yellow suspension was stirred at $0{ }^{\circ} \mathrm{C}$ for 2 hours before adding dropwise to a THF solution $(15 \mathrm{~mL})$ of $\mathrm{H}_{4}(\mathrm{mTPAd})(623 \mathrm{mg}, 0.42 \mathrm{mmol}, 1$ equiv.). An initial green colour was observed which afterwards turned red-brown once the addition was completed. The reaction was stirred for a further 16 hours and worked up in an identical manner to S2.4A. The purple solid $\left[\mathrm{NEt}_{4}\right]_{2}\left[\mathrm{Ce}_{2} \mathrm{Cl}_{2}\left(\mathrm{OSiMe}_{3}\right)_{4}\left(m \mathrm{mP}^{\mathrm{Ad}}\right)\right]$ 1-m-Ad was obtained (648 mg, 65\%).

${ }^{1} \mathrm{H}$ NMR $\left(500 \mathrm{MHz}\right.$, pyridine- $\left.\mathrm{d}_{5}\right) \delta_{\mathrm{H}}$ 7.31-7.28 (m, 2H, Ar-H), 7.20-7.17 (m, 2H, Ar-H), 7.13-7.11 (m, 2H, Ar-H), 7.09-7.06 (m, 2H, Ar-H), 7.02-6.98 (m, 2H, Ar- H), $6.90(\mathrm{~s}, 2 \mathrm{H}, \mathrm{Ar}-\mathrm{H}), 3.00\left(\mathrm{~m}, 16 \mathrm{H},\left({ }^{+} \mathrm{N}\left(\mathrm{CH}_{2} \mathrm{CH}_{3}\right)_{4}\right), 2.72-2.67(\mathrm{~m}\right.$, $12 \mathrm{H}), 2.44(\mathrm{~m}, 12 \mathrm{H}), 2.37-2.33(\mathrm{~m}, 12 \mathrm{H}), 2.30-2.24(\mathrm{~m}, 12 \mathrm{H}), 1.94-1.70(\mathrm{~m}, 24 \mathrm{H}), 1.14\left(\mathrm{~m}, 24 \mathrm{H},\left({ }^{+} \mathrm{N}\left(\mathrm{CH}_{2} \mathrm{CH}_{3}\right)_{4}\right)\right.$, $0.42\left(\mathrm{~s}, 36 \mathrm{H}, \mathrm{OSiMe}_{3}\right)$. 
${ }^{13}$ C DEPTQ NMR (126 MHz, pyridine- $d_{5}$ ) $\delta_{\mathrm{C}} 155.9$ (q, ArC-O), 129.9 (ArC-H), 129.1 (ArC-H), 128.3 (ArC-H), 127.9 (ArC-H), 126.2 (ArC-H), 117.7 (ArC-H), $53.0\left({ }^{+} \mathrm{N}_{(}\left(\mathrm{CH}_{2} \mathrm{CH}_{3}\right)_{4}\right), 42.2,41.5,41.3\left(-\mathrm{CH}_{2}\right), 38.3,38.1,37.90\left(-\mathrm{CH}_{2}\right), 30.5$, $30.0\left(-\mathrm{CH}_{3}\right), 29.8,8.0\left({ }^{+} \mathrm{N}\left(\mathrm{CH}_{2} \mathrm{CH}_{3}\right)_{4}\right), 2.49\left(\mathrm{CH}_{3}, \mathrm{OSiMe}_{3}\right)$.

${ }^{29} \mathrm{Si}$ NMR (99 MHz, pyridine- $\mathrm{d}_{5}$ ) $\delta_{\mathrm{Si}}-7.59$

Elemental analysis ([NEt $\left.]_{2}\left[\mathrm{Ce}_{2} \mathrm{Cl}_{2}\left(\mathrm{OSiMe}_{3}\right)_{4}\left(m \mathrm{mP}^{\mathrm{Ad}}\right)(\mathrm{THF})_{2}\right]\right): \mathrm{C} 61.82 \%, \mathrm{H} 8.25 \%, \mathrm{~N} 1.29 \%$ calculated; $\mathrm{C} 62.21 \%$, H $8.92 \%$, N $1.16 \%$ found.

Figure S15: ${ }^{1} \mathrm{H}$ NMR spectrum of $\left[\mathrm{NEt}_{4}\right]_{2}\left[\mathrm{Ce}_{2} \mathrm{Cl}_{2}\left(\mathrm{OSiMe}_{3}\right)_{4}\left(m T P^{\mathrm{Ad}}\right)\right]$ in $\mathrm{C}_{5} \mathrm{D}_{5} \mathrm{~N}$. 


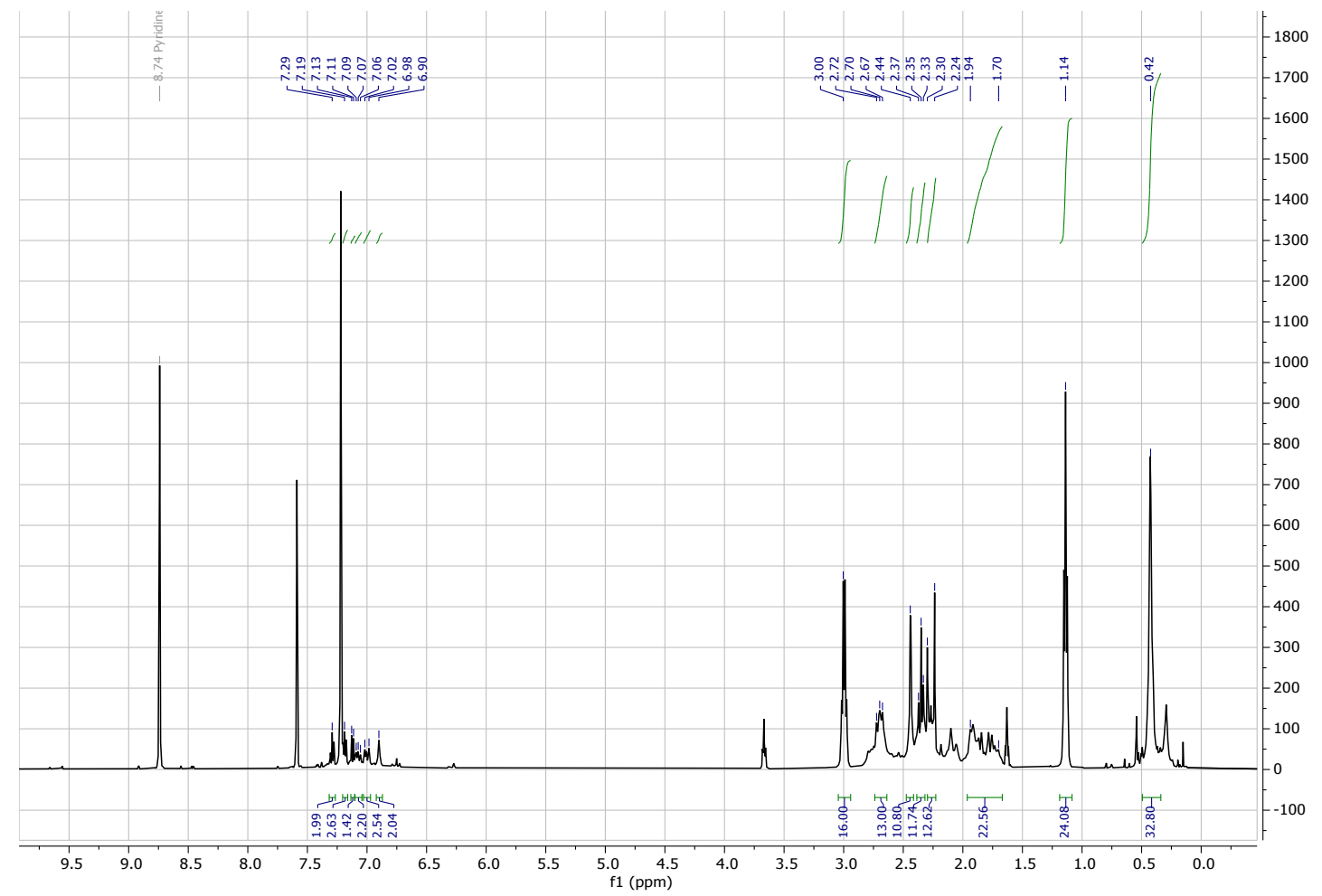

Figure S16: ${ }^{13} \mathrm{C}$ DEPTQ NMR spectrum of $\left[\mathrm{NEt}_{4}\right]_{2}\left[\mathrm{Ce}_{2} \mathrm{Cl}_{2}\left(\mathrm{OSiMe}_{3}\right)_{4}\left(p T P^{\mathrm{Ad}}\right)\right]$ in $\mathrm{C}_{5} \mathrm{D}_{5} \mathrm{~N}$.

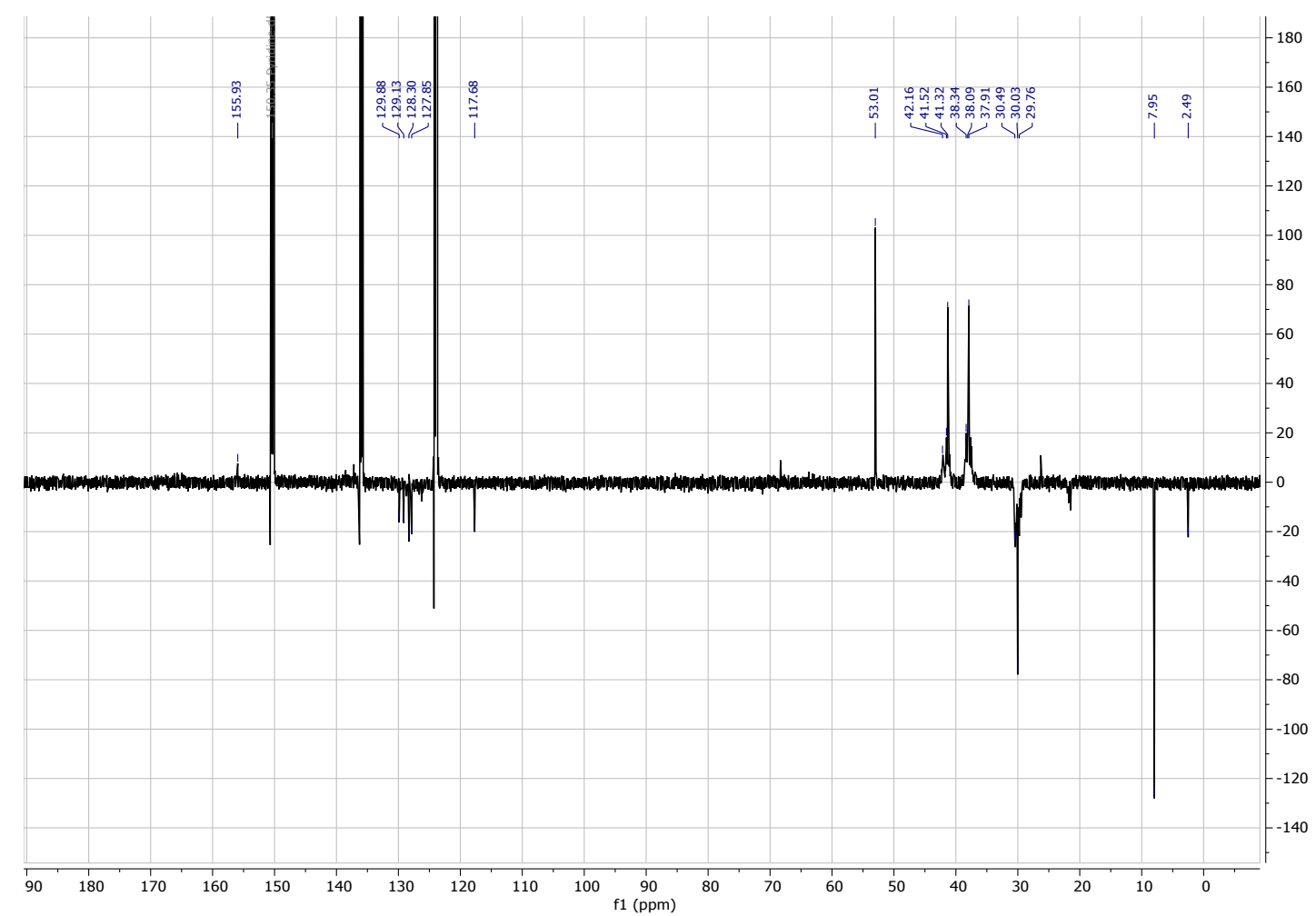

$\mathrm{S2.8}\left[\mathrm{NEt}_{4}\right]_{2}\left[\mathrm{Ce}_{2}\left(\mathrm{OSiMe}_{3}\right)_{6}\left(p \mathrm{TP}^{\mathrm{Ad}}\right)\right]$ 

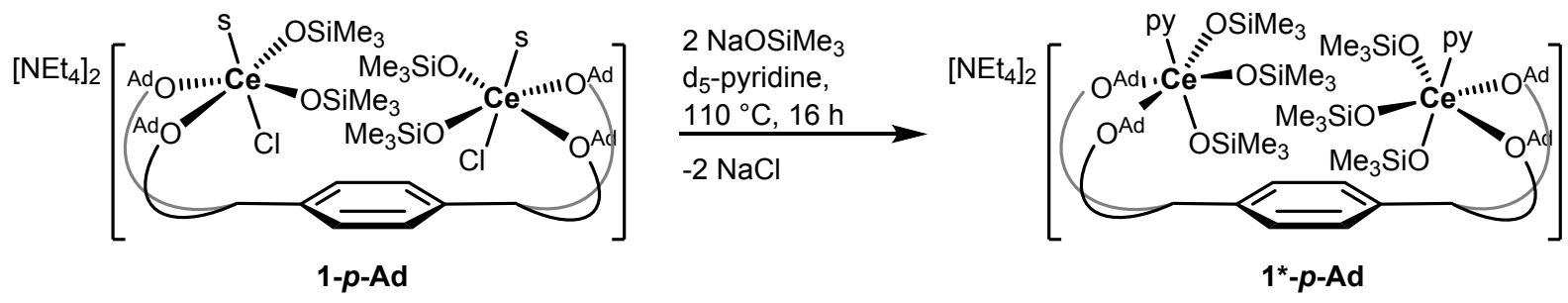

Equation S9: Synthesis of $\left[\mathrm{NEt}_{4}\right]_{2}\left[\mathrm{Ce}_{2}\left(\mathrm{OSiMe}_{3}\right)_{6}\left(p \mathrm{TPt}^{\mathrm{t}}\right)\right]$ from 2-p- ${ }^{\mathrm{t}} \mathrm{Bu}$.

A J. Young's tap NMR tube was charged with a $d_{5}$-pyridine solution $(0.6 \mathrm{~mL})$ of 1-p-Ad $(25 \mathrm{mg}, 0.01 \mathrm{mmol}, 1$ equiv.) and sodium trimethylsiloxide $(2.0 \mathrm{mg}, 0.02 \mathrm{mmol}, 2$ equiv.). Once sealed, the solution was heated at 110 ${ }^{\circ} \mathrm{C}$ for 16 hours. No change was observed in the ${ }^{1} \mathrm{H}$ NMR spectrum, however a few dark crystals grew from the concentrated solution, identified by X-ray crystallography as $1 *$-p-Ad.

\section{$\mathrm{S2.9}\left[\mathrm{NEt}_{4}\right]_{2}\left[\mathrm{Ce}_{2} \mathrm{Cl}_{4}(\mathrm{ODipp})_{2}\left(p \mathrm{TPt}^{\mathrm{t}}\right)\right]$}
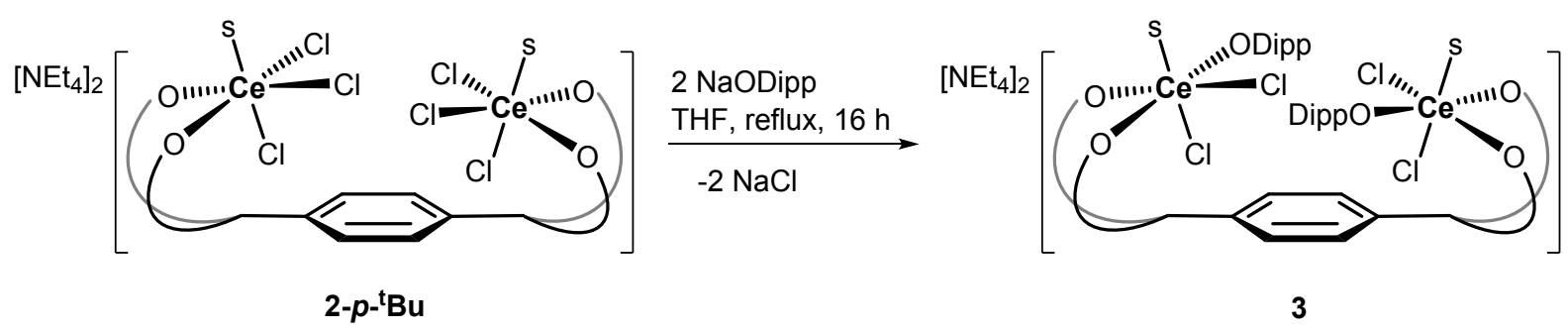

Equation S10: Synthesis of $\left[\mathrm{NEt}_{4}\right]_{2}\left[\mathrm{Ce}_{2} \mathrm{Cl}_{4}(\mathrm{ODipp})_{2}\left(p \mathrm{TP}^{\mathrm{t}}\right)\right]$.

To a purple THF solution $(20 \mathrm{~mL})$ of $\left[\mathrm{NEt}_{4}\right]_{2}\left[\mathrm{Ce}_{2} \mathrm{Cl}_{6}\left(p T \mathrm{Pt}^{\mathrm{t}}\right)\right](1.00 \mathrm{~g}, 0.61 \mathrm{mmol}, 1$ equiv.) was added, with stirring, a clear THF solution ( $20 \mathrm{~mL}$ ) of sodium 2,6-diisopropylphenoxide ( $243 \mathrm{mg}, 1.22 \mathrm{mmol}, 2$ equiv.). The resulting purple solution was heated under reflux for 16 hours before allowing to cool to room temperature, centrifuged, filtered via cannula and the solids extracted with THF $(2 \times 10 \mathrm{~mL})$. The filtrates were combined, solvent was evaporated under reduced pressure and the crude product dried yielding $\left[\mathrm{NEt}_{4}\right]_{2}\left[\mathrm{Ce}_{2} \mathrm{Cl}_{4}(\mathrm{ODipp})_{2}\left(p \mathrm{TP}^{\mathrm{t}}\right)\right] 3(910$ $\mathrm{mg}, 76 \%)$ as a purple solid.

${ }^{1} \mathrm{H}$ NMR $\left(500 \mathrm{MHz}\right.$, pyridine- $\left.\mathrm{d}_{5}\right) \delta_{\mathrm{H}} 7.96(\mathrm{~d}, \mathrm{~J}=2.5 \mathrm{~Hz}, 2 \mathrm{H}), 7.90(\mathrm{~d}, \mathrm{~J}=2.5 \mathrm{~Hz}, 2 \mathrm{H}), 7.54(\mathrm{~s}, 4 \mathrm{H}$, spacer), 7.41 (d, J $=2.5 \mathrm{~Hz}, 2 \mathrm{H}$ ), 7.35 (s, 2H, benzylic C-H), 7.27 (d, J = 7.7 Hz, 2H, Dipp), 7.19 (d, J = 7.7 Hz, 2H, Dipp), 7.17 (d, J = $2.5 \mathrm{~Hz}, 2 \mathrm{H}$ ), $6.87(\mathrm{t}, \mathrm{J}=7.7 \mathrm{~Hz}, 1 \mathrm{H}, \operatorname{Dipp}), 6.80$ (t, J = 7.7 Hz, 1H, Dipp), 4.17 (sept, J = 6.6 Hz, 2H, Dipp), 3.80 (sept, J = 6.6 Hz, 2H, Dipp), $3.33\left(\mathrm{q}, 16 \mathrm{H},\left({ }^{+} \mathrm{N}\left(\mathrm{C}_{2} \mathrm{CH}_{3}\right)_{4}\right), 1.76\left(\mathrm{~s}, 18 \mathrm{H},{ }^{\mathrm{t} B u}\right), 1.71\left(\mathrm{~s}, 18 \mathrm{H},{ }^{\mathrm{t} B u}\right), 1.34(\mathrm{~d}, \mathrm{~J}=6.6 \mathrm{~Hz}\right.$, $12 \mathrm{H}$, Dipp), 1.33 (s, 18H, $\left.{ }^{\mathrm{t}} \mathrm{Bu}\right), 1.30\left(\mathrm{~s}, 18 \mathrm{H},{ }^{\mathrm{t} B u}\right), 1.23\left(\mathrm{t}, 24 \mathrm{H},\left({ }^{+} \mathrm{N}\left(\mathrm{CH}_{2} \mathrm{CH}_{3}\right)_{4}\right)\right.$.

${ }^{13} \mathrm{C}$ DEPTQ NMR (126 MHz, pyridine- $d_{5}$ ) $\delta_{\mathrm{C}} 169.4$ (q, ArC-O), 168.9 (q, ArC-O), 167.8 (q, ArC-O), 165.7 (q, ArC-O), 152.8 ( $q, \operatorname{ArC}), 143.7$ ( $q, \operatorname{ArC}), 143.1$ ( $q, \operatorname{ArC}), 139.9$ ( $q, \operatorname{ArC}), 139.2$ (q, ArC), $138.4(q, \operatorname{ArC}), 138.1(q, \operatorname{ArC}), 137.8$ (q, $\operatorname{ArC}), 137.1$ (q, ArC), 134.4 (q, ArC), 134.2 (q, ArC), 130.1 (ArC-H), 126.5 (ArC-H), $122.9(\operatorname{ArC}-\mathrm{H}), 122.8(\operatorname{ArC}-$ H), 119.3 (ArC-H), 119.1 (ArC-H), $118.1\left(\right.$ ArC-H), $53.2\left({ }^{+} \mathrm{N}\left(\mathrm{CH}_{2} \mathrm{CH}_{3}\right)_{4}\right), 46.1$ (benzylic C-H), $36.2\left(\mathrm{q},{ }^{\mathrm{t}} \mathrm{Bu}\right), 36.1$ (q, $\left.{ }^{t} \mathrm{Bu}\right), 35.1\left(\mathrm{q},{ }^{\mathrm{t}} \mathrm{Bu}\right), 34.9\left(\mathrm{q},{ }^{\mathrm{t}} \mathrm{Bu}\right), 33.3\left(\mathrm{CH}_{3},{ }^{\mathrm{t}} \mathrm{Bu}\right), 33.0\left(\mathrm{CH}_{3},{ }^{\mathrm{t} B u}\right), 32.4\left(\mathrm{CH}_{3},{ }^{\mathrm{t} B u}\right), 31.8\left(\mathrm{CH}_{3},{ }^{\mathrm{t} B u}\right), 28.4\left(\mathrm{CH}(\mathrm{CH})_{2}\right)$, $28.1\left(\mathrm{CH}\left(\mathrm{CH}_{3}\right)_{2}\right), 25.9\left(\mathrm{CH}\left(\mathrm{CH}_{3}\right)_{2}\right), 25.6\left(\mathrm{CH}\left(\mathrm{CH}_{3}\right)_{2}\right), 25.0\left(\mathrm{CH}\left(\mathrm{CH}_{3}\right)_{2}\right), 8.0\left({ }^{+} \mathrm{N}\left(\mathrm{CH}_{2} \mathrm{CH}_{3}\right)_{4}\right)$.

Elemental analysis ([NEt $\left.]_{2}\left[\mathrm{Ce}_{2} \mathrm{Cl}_{4}(\mathrm{ODipp})_{2}\left(p \mathrm{TP}^{\mathrm{t}}\right)(\mathrm{THF})_{2}\right]\right): \mathrm{C} 64.04 \%, \mathrm{H} 8.45 \%, \mathrm{~N} \mathrm{1.33 \%}$ calculated; C 64.07\%, $\mathrm{H}$ $8.35 \%, \mathrm{~N} 1.09 \%$ found. 
Figure S17: ${ }^{1} \mathrm{H}$ NMR spectra of $\left[\mathrm{NEt}_{4}\right]_{2}\left[\mathrm{Ce}_{2} \mathrm{Cl}_{4}(\mathrm{ODipp})_{2}\left(p T \mathrm{Pt}^{\mathrm{t}}\right)\right]$ in $\mathrm{C}_{5} \mathrm{D}_{5} \mathrm{~N}$.
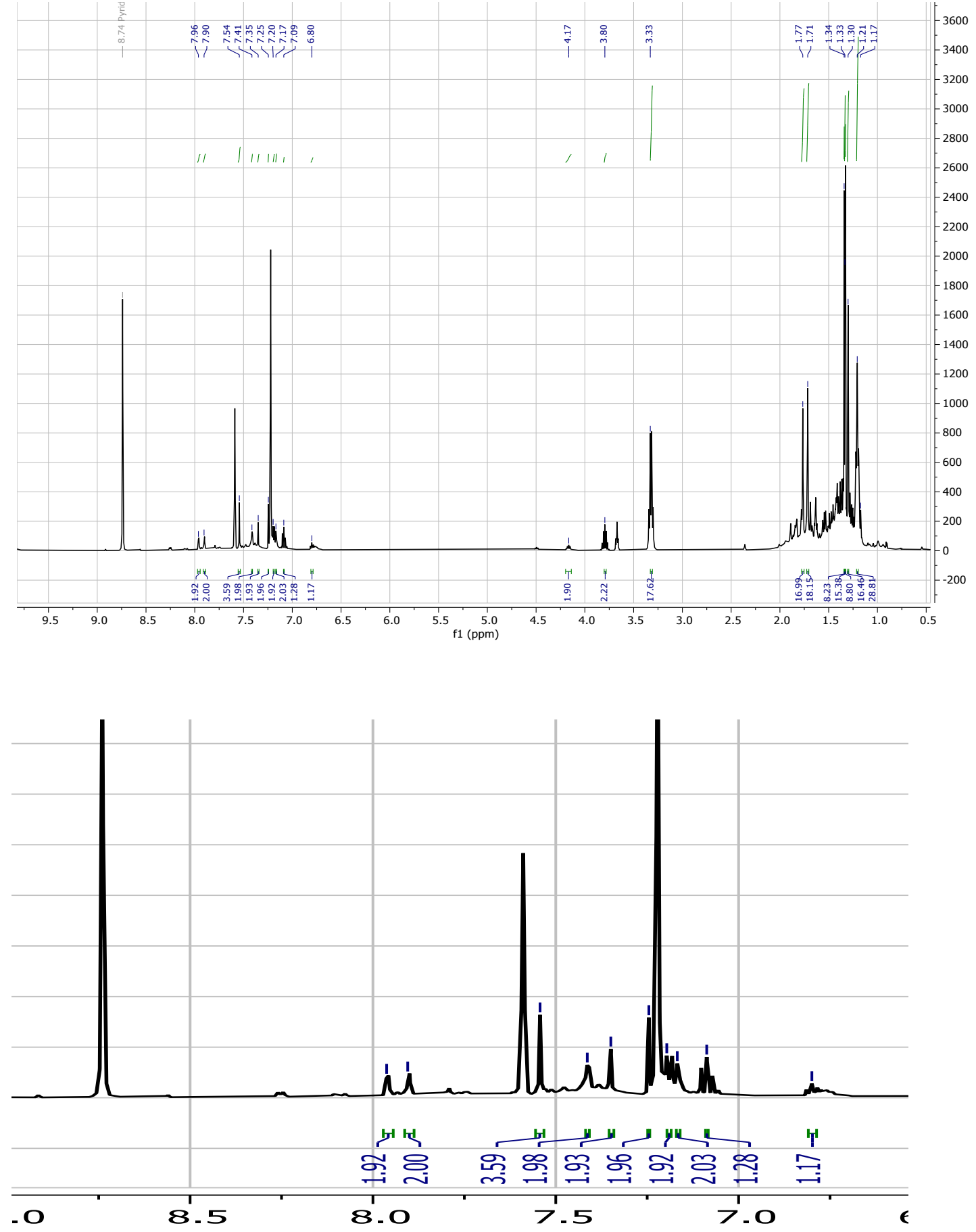
${ }^{1} \mathrm{H}-{ }^{1} \mathrm{H}$ NOESY spectrum of $\left[\mathrm{NEt}_{4}\right]_{2}\left[\mathrm{Ce}_{2} \mathrm{Cl}_{4}(\mathrm{ODipp})_{2}\left(p T P^{\mathrm{t}}\right)\right]$ in $\mathrm{C}_{5} \mathrm{D}_{5} \mathrm{~N}$. The only cross-peaks observable are that related to the $\mathrm{NEt}_{4}$ counterions - located at 3.3 and $1.2 \mathrm{ppm}$.

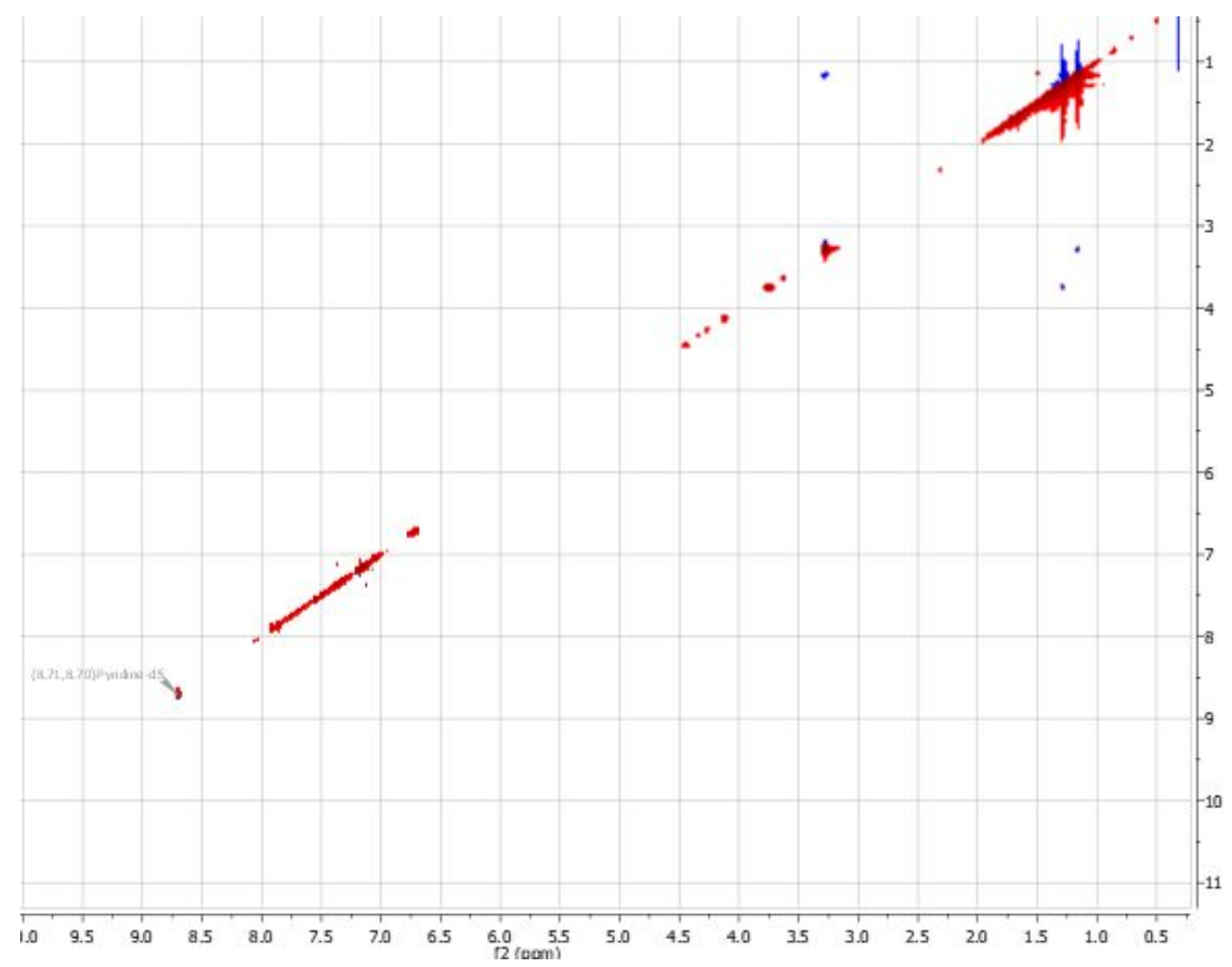

Figure S18: ${ }^{13} \mathrm{C}$ DEPTQ NMR spectrum of $\left[\mathrm{NEt}_{4}\right]_{2}\left[\mathrm{Ce}_{2} \mathrm{Cl}_{4}(\mathrm{ODipp})_{2}\left(p T \mathrm{Pt}^{\mathrm{t}}\right)\right]$ in $\mathrm{C}_{5} \mathrm{D}_{5} \mathrm{~N}$.

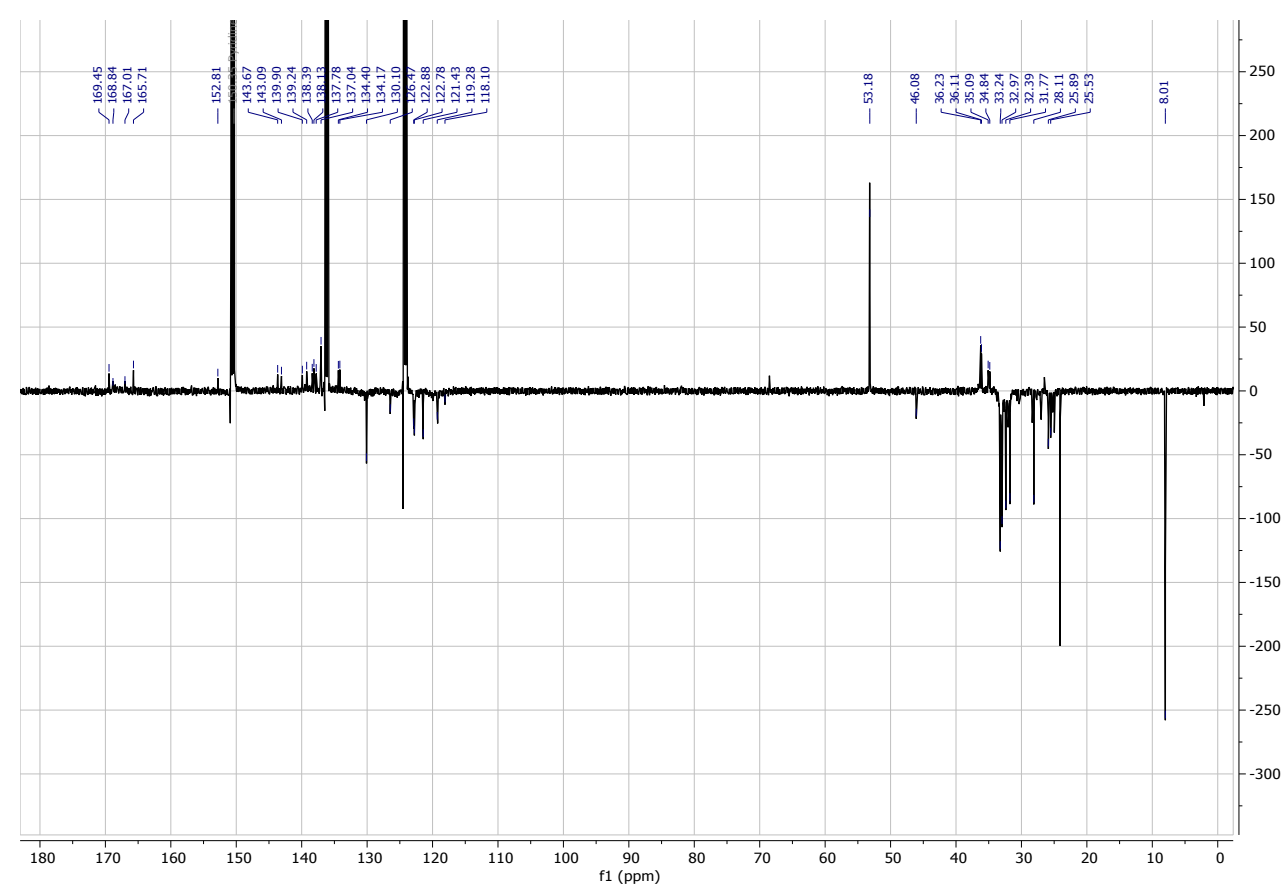




\section{S2.10 Synthesis of $\left[\mathrm{NEt}_{4}\right]_{2}\left[\mathrm{Ce}_{2} \mathrm{Cl}_{6}\left(m T e r T P^{\mathrm{t}}\right)\right]$}

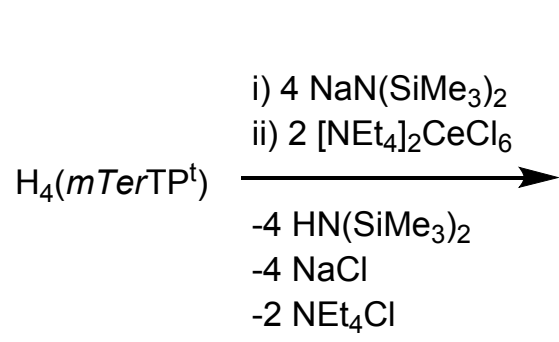

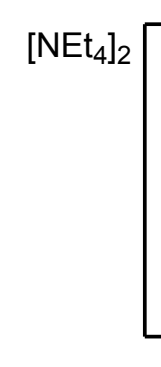

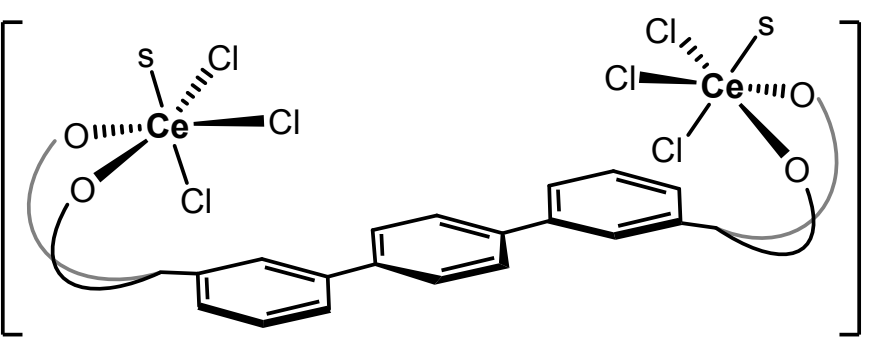

4

Equation S11: Synthesis of $\left[\mathrm{NEt}_{4}\right]_{2}\left[\mathrm{Ce}_{2} \mathrm{Cl}_{6}\left(m \operatorname{merTP}^{\mathrm{t}}\right)\right]$.

By analogous procedure to $\mathbf{S 2 . 3 .}$

$\mathrm{H}_{4}$ (mTerTPt) (269 mg, $0.25 \mathrm{mmol}, 1$ equiv.) and $\mathrm{NaN}\left(\mathrm{SiMe}_{3}\right)_{2}$ (183 mg, $1.00 \mathrm{mmol} 4$ equiv.) were dissolved in THF $(10 \mathrm{~mL})$ and the resulting clear solution stirred for 2 hours. $\left[\mathrm{NEt}_{4}\right]_{2} \mathrm{CeCl}_{6}(307 \mathrm{mg}, 0.50 \mathrm{mmol}, 2$ equiv.) was suspended in THF $(10 \mathrm{~mL})$ at $0{ }^{\circ} \mathrm{C}$ and to this was slowly added the $\mathrm{Na}_{4}\left(m T e r T P^{t}\right)$ solution, forming a purple solution which was stirred for 16 hours at room temperature. The mixture was centrifuged and cooled in an icebath, filtered via cannula whilst cold and the volatiles evaporated under reduced pressure. The crude product was dried, then triturated with hexane $(3 \times 10 \mathrm{~mL})$ before drying again, yielding $\left[\mathrm{NEt}_{4}\right]_{2}\left[\mathrm{Ce}_{2} \mathrm{Cl}_{6}\left(m T e r T \mathrm{P}^{\mathrm{t}}\right)\right] 4(351$ $\mathrm{mg}, 77 \%)$ as a purple solid.

${ }^{1} \mathrm{H}$ NMR $\left(500 \mathrm{MHz}\right.$, pyridine-d $\left.\mathrm{d}_{5}\right) \delta_{\mathrm{H}} 8.33(\mathrm{~s}, 1 \mathrm{H}, \mathrm{Ar}-\mathrm{H}), 8.25(\mathrm{~s}, 1 \mathrm{H}, \mathrm{Ar}-\mathrm{H}), 8.18(\mathrm{~s}, 1 \mathrm{H}, \mathrm{Ar}-\mathrm{H}), 7.83(\mathrm{~m}, 2 \mathrm{H}, \mathrm{Ar}-\mathrm{H})$, $7.72(\mathrm{~m}, 2 \mathrm{H}, \mathrm{Ar}-\mathrm{H}), 7.70(\mathrm{~m}, 2 \mathrm{H}, \mathrm{Ar}-\mathrm{H}), 7.49$ (s, 1H, Ar-H), $7.40(\mathrm{~s}, 1 \mathrm{H}, \mathrm{Ar}-\mathrm{H}), 7.38(\mathrm{~s}, 1 \mathrm{H}, \mathrm{Ar}-\mathrm{H}), 7.36(\mathrm{~m}, 2 \mathrm{H}, \mathrm{Ar}-\mathrm{H})$, $7.35(\mathrm{~m}, 2 \mathrm{H}, \mathrm{Ar}-\mathrm{H}), 7.31(\mathrm{~m}, 1 \mathrm{H}, \mathrm{Ar}-\mathrm{H}), 7.30(\mathrm{~m}, 1 \mathrm{H}, \mathrm{Ar}-\mathrm{H}), 7.28(\mathrm{~m}, 2 \mathrm{H}, \mathrm{Ar}-\mathrm{H}), 6.82(\mathrm{~s}, 2 \mathrm{H}$, benzylic C-H), $3.32(\mathrm{q}$, $16 \mathrm{H},\left({ }^{+} \mathrm{N}\left(\mathrm{CH}_{2} \mathrm{CH}_{3}\right)_{4}\right), 1.86\left(\mathrm{br} \mathrm{s}, 18 \mathrm{H},{ }^{\mathrm{t}} \mathrm{Bu}\right), 1.64(9 \mathrm{H}, \mathrm{tBu}), 1.49\left(\mathrm{~s}, 18 \mathrm{H},{ }^{\mathrm{t}} \mathrm{Bu}\right), 1.36\left(\mathrm{~s}, 9 \mathrm{H},{ }^{\mathrm{t}} \mathrm{Bu}\right), 1.33\left(\mathrm{~s}, 9 \mathrm{H},{ }^{\mathrm{t}} \mathrm{Bu}\right), 1.31$ $\left(\mathrm{s}, 9 \mathrm{H},{ }^{\mathrm{t}} \mathrm{Bu}\right), 1.19\left(\mathrm{t}, 24 \mathrm{H},\left({ }^{+} \mathrm{N}\left(\mathrm{CH}_{2} \mathrm{CH}_{3}\right)_{4}\right)\right.$.

${ }^{13} \mathrm{C}$ DEPTQ NMR (126 MHz, pyridine- $d_{5}$ ) $\delta_{\mathrm{C}} 168.2$ (q, ArC-O), 149.5 (q, ArC), 143.5 (q, ArC), 141.2 ( $\left.q, \operatorname{ArC}\right), 133.0$ (ArC-H), 129.3 (ArC-H), 128.8 (ArC-H), 128.5 (ArC-H), $127.6(\mathrm{ArC}-\mathrm{H}), 120.1(\mathrm{ArC}-\mathrm{H}), 53.0\left({ }^{+} \mathrm{N}\left(\mathrm{CH}_{2} \mathrm{CH}_{3}\right)_{4}\right), 44.4$ $\left(\mathrm{CH}_{3},{ }^{\mathrm{t}} \mathrm{Bu}\right), 36.1\left(\mathrm{q},{ }^{\mathrm{t}} \mathrm{Bu}\right), 36.0\left(\mathrm{q},{ }^{\mathrm{t}} \mathrm{Bu}\right), 34.9\left(\mathrm{q},{ }^{\mathrm{t}} \mathrm{Bu}\right), 33.1\left(\mathrm{CH}_{3},{ }^{\mathrm{t} B u}\right), 32.8\left(\mathrm{CH}_{3},{ }^{\mathrm{t} B u}\right), 30.9\left(\mathrm{CH}_{3},{ }^{\mathrm{t}} \mathrm{Bu}\right), 26.3\left(\mathrm{q},{ }^{\mathrm{t}} \mathrm{Bu}\right)$, $7.8\left({ }^{+} \mathrm{N}\left(\mathrm{CH}_{2} \mathrm{CH}_{3}\right)_{4}\right)$.

Elemental analysis $\left(\left[\mathrm{NEt}_{4}\right]_{2}\left[\mathrm{Ce}_{2} \mathrm{Cl}_{6}\left(m \operatorname{merTP}^{\mathrm{t}}\right)(\mathrm{THF})_{2}\right]\right): \mathrm{C} 60.55 \%, \mathrm{H} 8.65 \%, \mathrm{~N} 1.40 \%$ calculated; $\mathrm{C} 60.44 \%, \mathrm{H}$ $8.76 \%$, N $1.39 \%$ found. 
Figure S19: ${ }^{1} \mathrm{H}$ NMR spectrum of $\left[\mathrm{NEt}_{4}\right]_{2}\left[\mathrm{Ce}_{2} \mathrm{Cl}_{6}\left(m T e r T P^{t}\right)\right]$ in $\mathrm{C}_{5} \mathrm{D}_{5} \mathrm{~N}$.

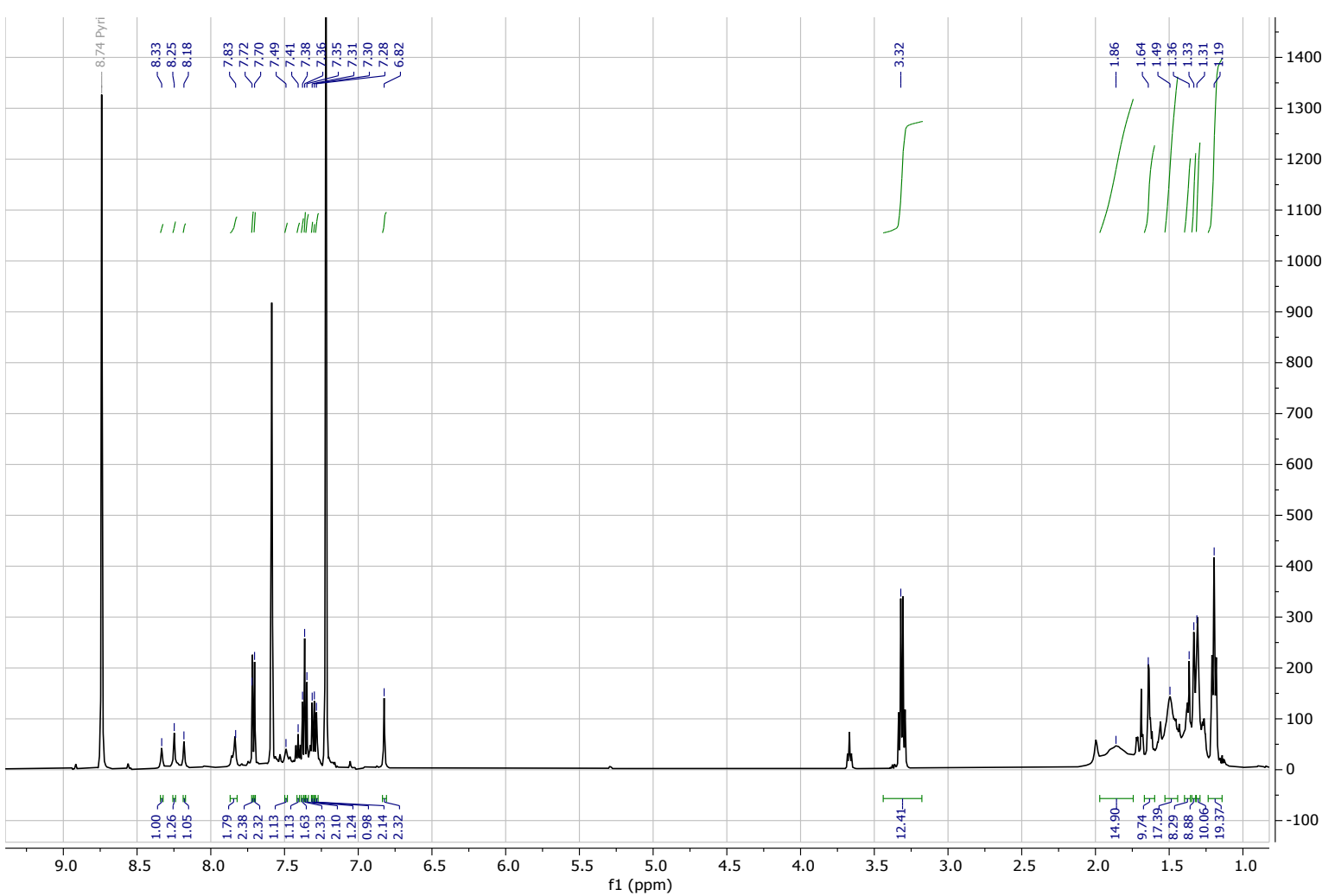

Figure S20: ${ }^{13} \mathrm{C}$ DEPTQ NMR spectrum of $\left[\mathrm{NEt}_{4}\right]_{2}\left[\mathrm{Ce}_{2} \mathrm{Cl}_{6}\left(m T e r T P^{t}\right)\right]$ in $\mathrm{C}_{5} \mathrm{D}_{5} \mathrm{~N}$.

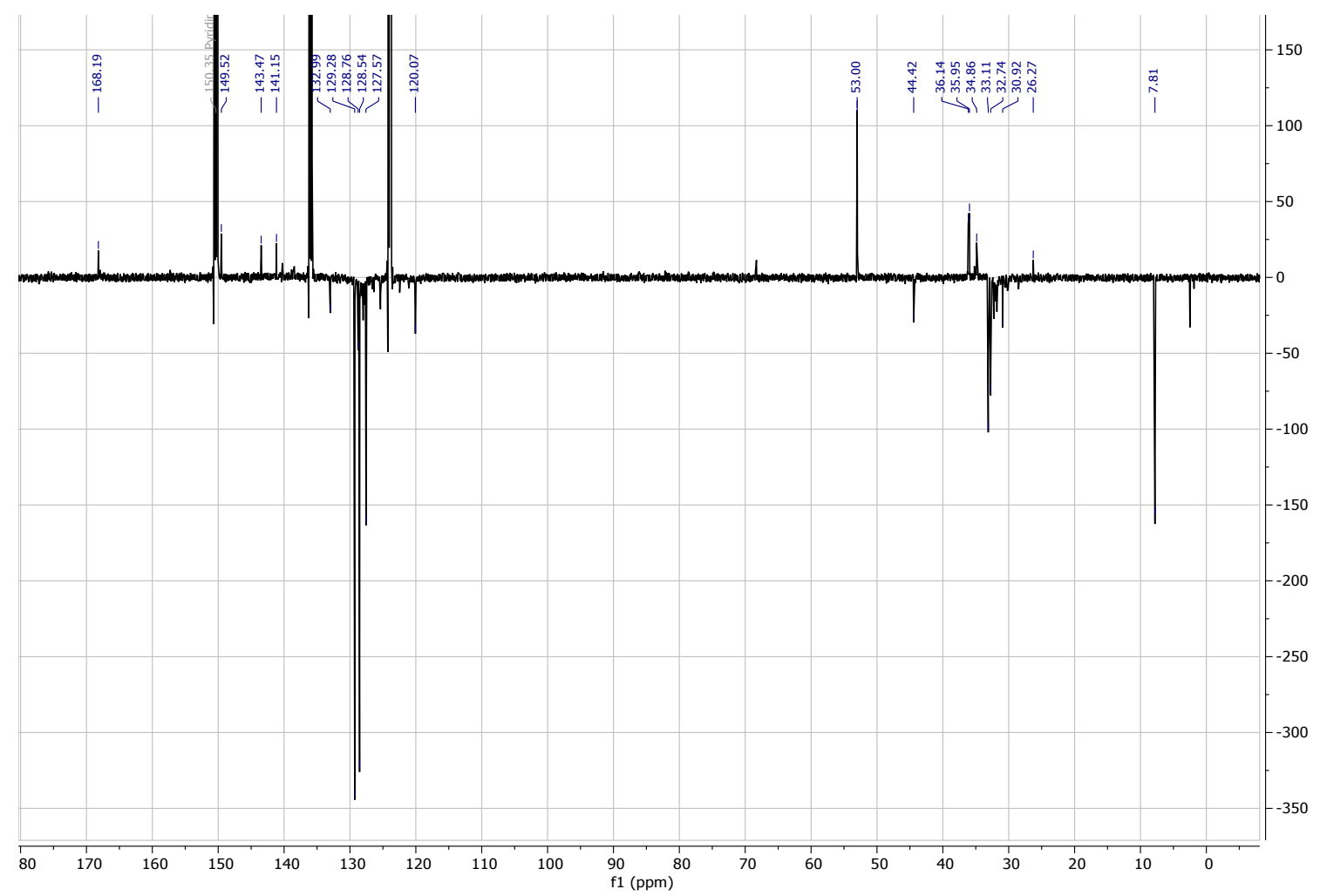




\section{S2.11 Synthesis of neutral di-Ce(IV) complex}

\begin{tabular}{|c|c|c|}
\hline \multirow{4}{*}{$\left(\mathrm{NH}_{4}\right)_{2} \mathrm{Ce}\left(\mathrm{NO}_{3}\right)_{6}+6 \mathrm{NaOSiEt}_{3}$} & $\begin{array}{l}\text { THF } \\
\text { r.t., } 16 \mathrm{~h}\end{array}$ & \multirow{2}{*}{$\mathrm{Ce}\left(\mathrm{OSiEt}_{3}\right)_{4}$} \\
\hline & $-6 \mathrm{NaNO}_{3}$ & \\
\hline & $-2 \mathrm{HOSiEt}_{3}$ & \\
\hline & $-2 \mathrm{NH}_{3}$ & \\
\hline
\end{tabular}

Equation S12: Synthesis of $\mathrm{Ce}\left(\mathrm{OSiEt}_{3}\right)_{4}$.

Prepared using the method described by Anwander et al. and Evans et al. ${ }^{8,9}$ A colourless THF solution ( $\left.2 \mathrm{~mL}\right)$ of $\mathrm{NaOSiEt}_{3}$ (212.4 mg, $1.38 \mathrm{mmol}, 6$ equiv.) was added dropwise to a vigorously stirring orange THF suspension ( 2 $\mathrm{mL}$ ) of $\left(\mathrm{NH}_{4}\right)_{2} \mathrm{Ce}\left(\mathrm{NO}_{3}\right)_{6}(125.8 \mathrm{mg}, 0.230 \mathrm{mmol}, 1$ equiv.). The mixture became a cloudy yellow suspension and gas evolution was observed for about 1 hour. Stirring was continued for 16 hours after which the white suspension was centrifuged, filtered and the filtrate evaporated to dryness by heating under vacuum at $40{ }^{\circ} \mathrm{C}$ for 5 hours. A sticky yellow solid was obtained (95 $\mathrm{mg}, 62 \%)$.

${ }^{1} \mathrm{H}$ NMR $\left(500 \mathrm{MHz}\right.$, pyridine- $\left.\mathrm{d}_{5}\right) \delta_{\mathrm{H}} 1.11(\mathrm{t}, J=7.5 \mathrm{~Hz}, 36 \mathrm{H}), 0.71(\mathrm{q}, J=7.5 \mathrm{~Hz}, 24 \mathrm{H})$.

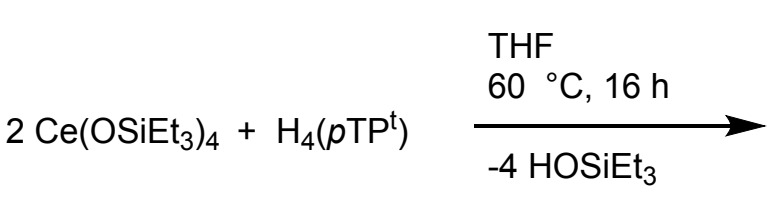

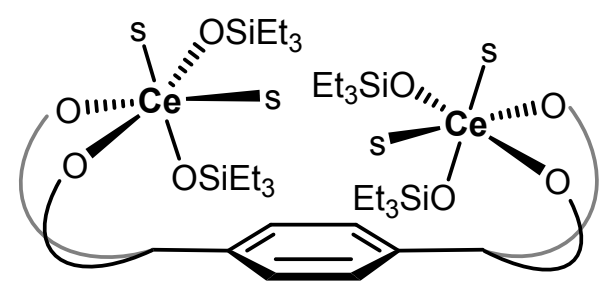

5

Equation S13: Synthesis of $\left[\mathrm{Ce}\left(\mathrm{OSiEt}_{3}\right)_{4}\left(p \mathrm{PP}^{\mathrm{t}}\right)\right] ; \mathrm{s}=$ solvent

In a Schlenk flask, $\mathrm{H}_{4}\left(p \mathrm{TPt}^{\mathrm{t}}\right)(55 \mathrm{mg}, 0.060 \mathrm{mmol}, 1$ equiv.) was dissolved in THF $(10 \mathrm{~mL})$. To this clear, stirring solution, a pale yellow THF solution $(3 \mathrm{~mL})$ of $\mathrm{Ce}\left(\mathrm{OSiEt}_{3}\right)_{4}(80 \mathrm{mg}, 0.120 \mathrm{mmol}, 2$ equiv.) was added dropwise. The mixture immediately turned a deep purple colour, and the reaction was heated at $60^{\circ} \mathrm{C}$ with stirring for 16 hours. Volatiles were evaporated under reduced pressure and the residue washed with hexane $(2 \times 5 \mathrm{~mL})$ before drying at $50{ }^{\circ} \mathrm{C}$ for $6 \mathrm{~h}$. [Ce(OSiEt $\left.)_{4}\left(\mathrm{pTPt}^{\mathrm{t}}\right)\right] \mathbf{5}$ was obtained as a purple solid (70 $\left.\mathrm{mg}, 68 \%\right)$.

${ }^{1} \mathrm{H}$ NMR $\left(500 \mathrm{MHz}\right.$, pyridine- $\left.\mathrm{d}_{5}\right) \delta_{\mathrm{H}} 7.94(2 \mathrm{H}, \mathrm{Ar}-\mathrm{H}), 7.44(2 \mathrm{H}, \mathrm{Ar}-\mathrm{H}), 7.30(2 \mathrm{H}, \mathrm{Ar}-\mathrm{H}), 7.08(\mathrm{~s}, 4 \mathrm{H}$, spacer Ar-H), 7.02 $(2 \mathrm{H}, \mathrm{Ar}-\mathrm{H}), 1.44\left(\mathrm{~s}, 36 \mathrm{H},{ }^{\mathrm{t} B u}\right), 1.35\left(\mathrm{~s}, 36 \mathrm{H},{ }^{\mathrm{t} B u}\right), 1.13(\mathrm{t}, J=7.5 \mathrm{~Hz}, 36 \mathrm{H}), 0.73(\mathrm{q}, J=7.5 \mathrm{~Hz}, 24 \mathrm{H})$.

${ }^{13} \mathrm{C}$ DEPTQ NMR (126 MHz, pyridine- $d_{5}$ ) $\delta_{\mathrm{C}} 166.8$ (q, ArC-O), 145.4 (q, ArC), 140.9 ( $\left.q, \operatorname{ArC}\right), 137.9$ ( $\left.q, \operatorname{ArC}\right), 134.3$ (q, ArC), 130.1 (ArC-H), 129.7 (ArC-H), 125.5 (ArC-H), 120.5 (ArC-H), 44.0 (benzylic C-H), 36.0 (q, t' Bu), 35.6 (q, $\left.{ }^{t} \mathrm{Bu}\right), 34.9\left(\mathrm{q},{ }^{\mathrm{t}} \mathrm{Bu}\right), 32.7\left(\mathrm{CH}_{3},{ }^{\mathrm{t}} \mathrm{Bu}\right), 31.2\left(\mathrm{CH}_{3},{ }^{\mathrm{t}} \mathrm{Bu}\right), 26.3\left(\mathrm{q},{ }^{\mathrm{t}} \mathrm{Bu}\right), 8.4\left(\mathrm{OSiCH}_{2} \mathrm{CH}_{3}\right), 8.2\left(\mathrm{OSiCH}_{2} \mathrm{CH}_{3}\right), 7.7\left(\mathrm{OSiCH}_{2} \mathrm{CH}_{3}\right)$, $7.2\left(\mathrm{OSiCH}_{2} \underline{\mathrm{C}} \mathrm{H}_{3}\right)$.

${ }^{29} \mathrm{Si}$ NMR $\left(99 \mathrm{MHz}\right.$, pyridine- $\left.\mathrm{d}_{5}\right) \delta_{\mathrm{si}} 13.09$.

Elemental analysis ([Ce $\left.\left.2\left(\mathrm{OSiEt}_{3}\right)_{4}\left(\mathrm{pTP}^{\mathrm{t}}\right)(\mathrm{THF})_{4}\right]\right): \mathrm{C} 62.05 \%, \mathrm{H} 9.54 \%, \mathrm{~N} 0.00 \%$ calculated; C $62.37 \%, \mathrm{H} 9.81 \%, \mathrm{~N}$ $0.00 \%$ found. 
Figure S21: ${ }^{1} \mathrm{H}$ NMR spectrum of $\left[\mathrm{Ce}\left(\mathrm{OSiEt}_{3}\right)_{4}\left(p \mathrm{PP}^{t}\right)\right]$ in $\mathrm{C}_{5} \mathrm{D}_{5} \mathrm{~N}$. Residual THF is also present.

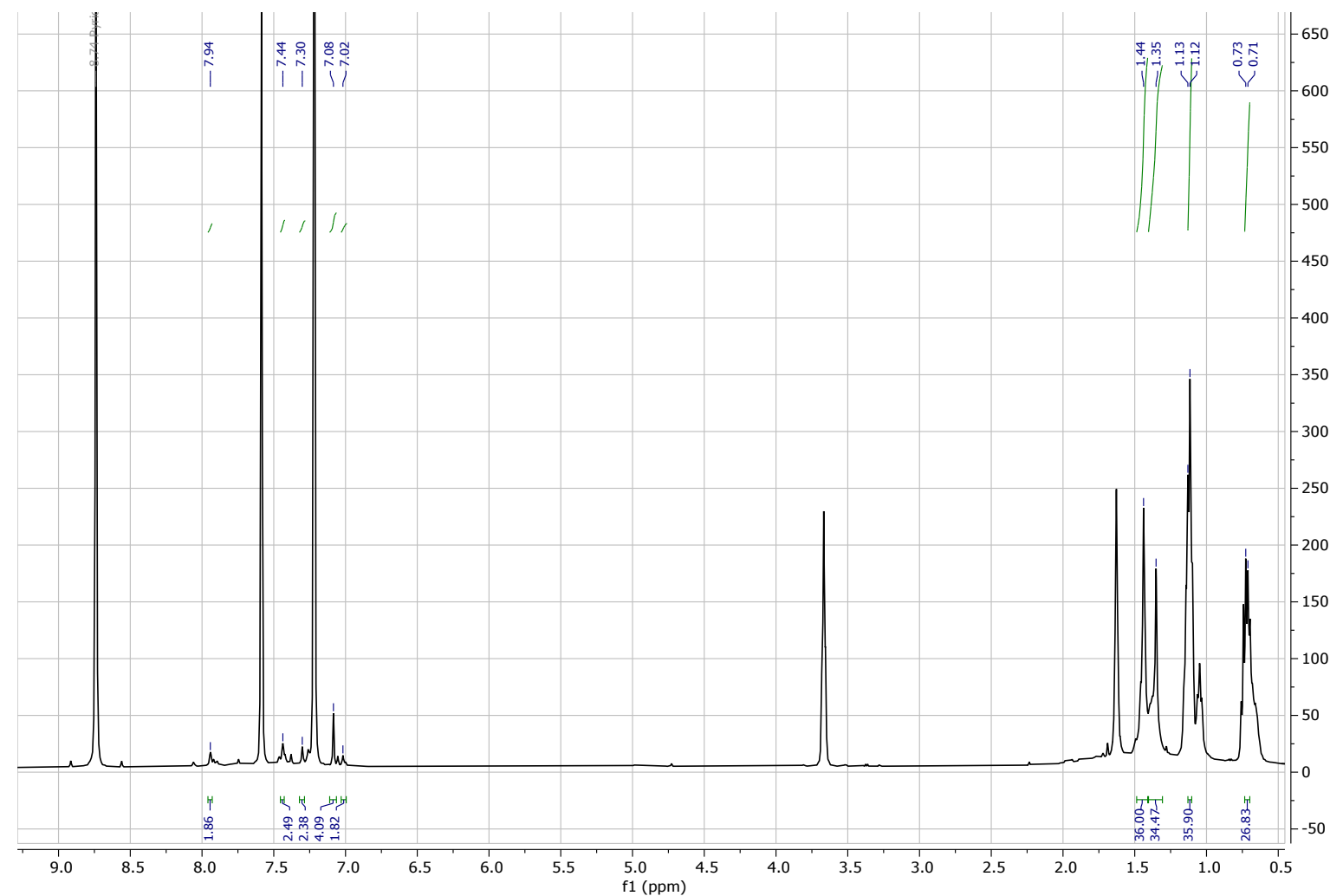

Figure S22: ${ }^{13} \mathrm{C}$ DEPTQ NMR spectrum of $\left[\mathrm{Ce}\left(\mathrm{OSiEt}_{3}\right)_{4}\left(p T \mathrm{Pt}^{\mathrm{t}}\right)\right]$ in $\mathrm{C}_{5} \mathrm{D}_{5} \mathrm{~N}$.

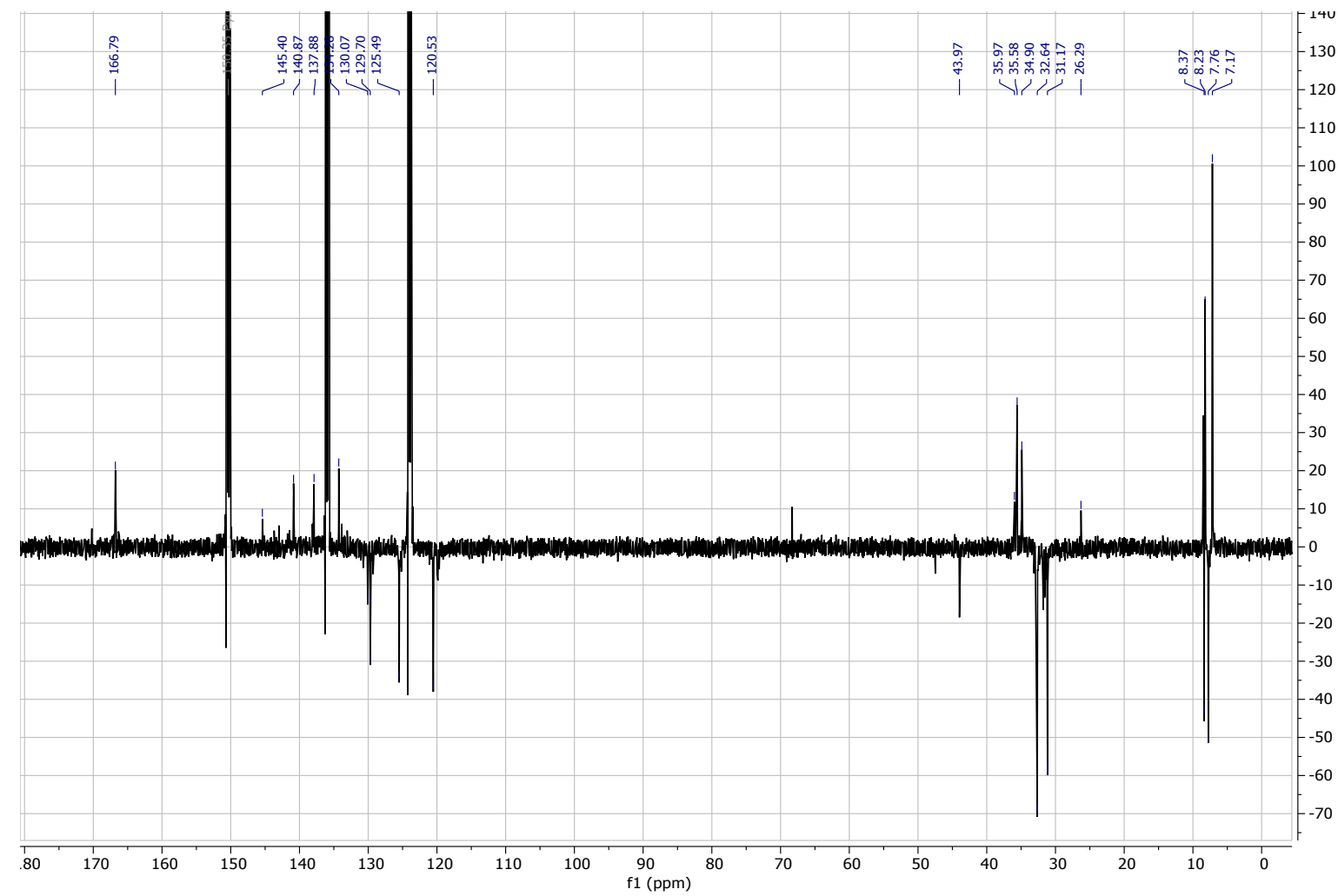




\section{S2.12 Cyclic voltammetry studies}

Measurements were conducted in THF using $0.1 \mathrm{M}\left[\mathrm{nBu}_{4} \mathrm{~N}\right]\left[\mathrm{PF}_{6}\right]$ as a supporting electrolyte. The mass of complex used was that required to form a $0.01 \mathrm{mM}$ solution.

Table S1: Reduction potentials of selected di-Ce(IV) complexes.

\begin{tabular}{|c|c|}
\hline Complex & $E_{\mathrm{pc}} \mathrm{vs} \mathrm{Fc/Fc^{+ }}$ \\
\hline & $\mathrm{V}$ \\
\hline $1-p-{ }^{\mathrm{t}} \mathrm{Bu}$ & -2.47 \\
\hline $2-p-{ }^{\mathrm{t}} \mathrm{Bu}$ & -1.98 \\
\hline 3 & -1.18 \\
\hline
\end{tabular}

Figure S23: Cyclic voltammograms of selected di-Ce(IV) complexes. $\rightarrow=$ scan direction
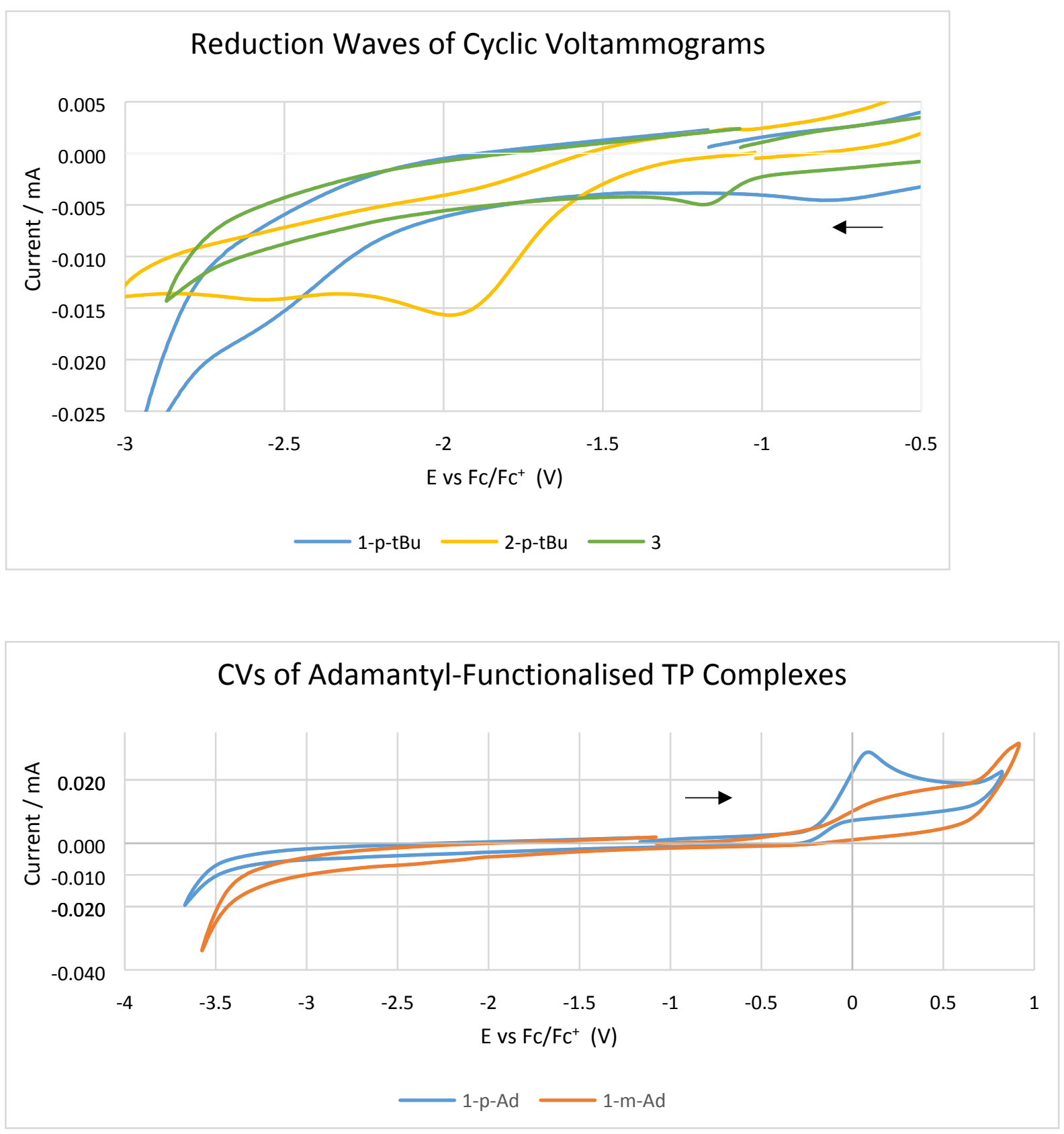

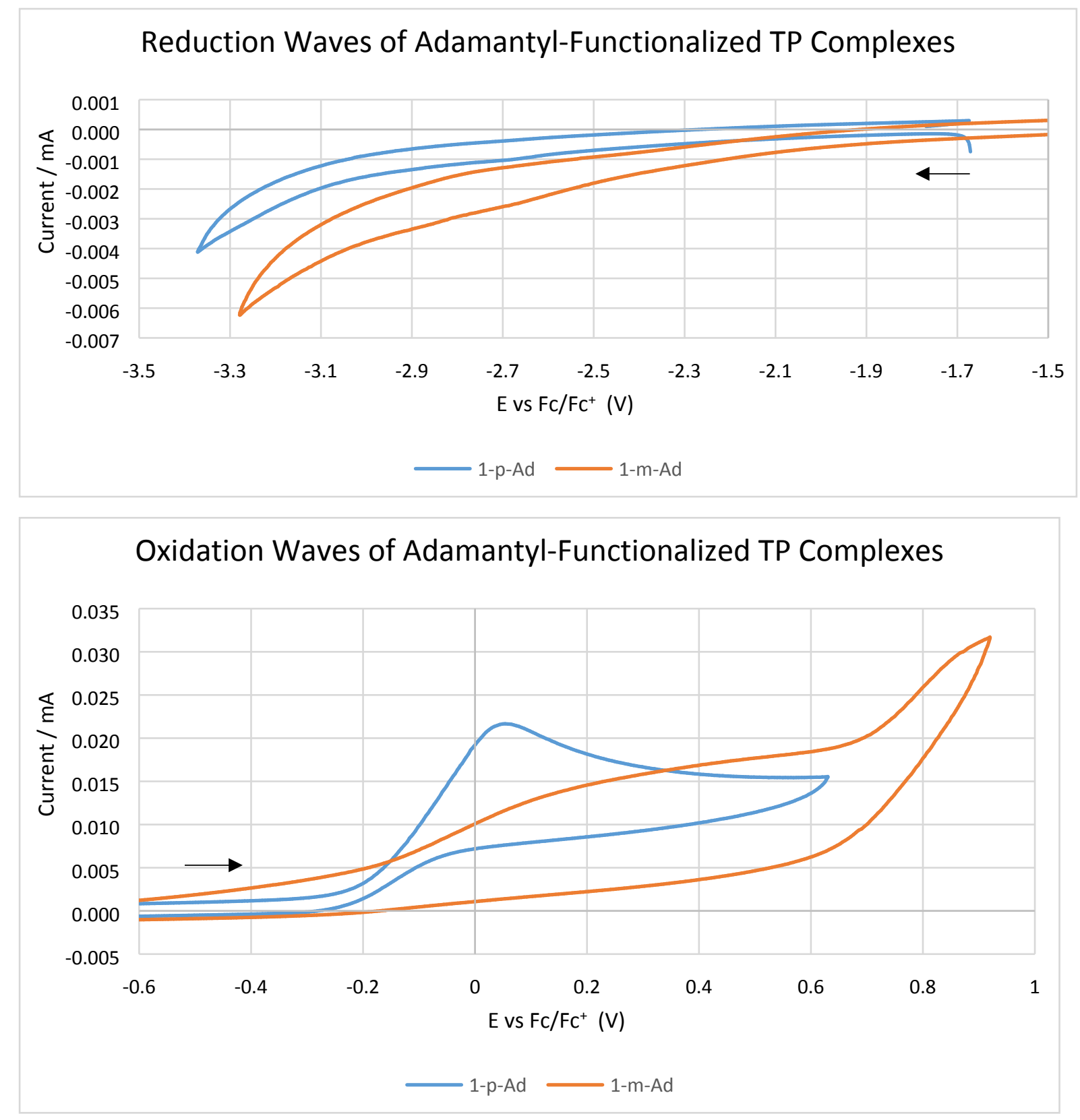


\section{S2.13 UV-Vis}

All UV-Vis spectra were recorded in THF, using concentrations of $3 \times 10^{-5} \mathrm{M}$ for 1-p-t $\mathrm{Bu}, \mathbf{1}-\boldsymbol{m}$ - $^{\mathrm{t}} \mathrm{Bu}, \mathbf{2}-\boldsymbol{p}$ -

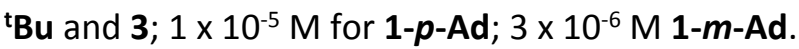

Figure S24: UV-Vis spectra of selected di-Ce(IV) complexes.

\section{UV-Vis spectra}

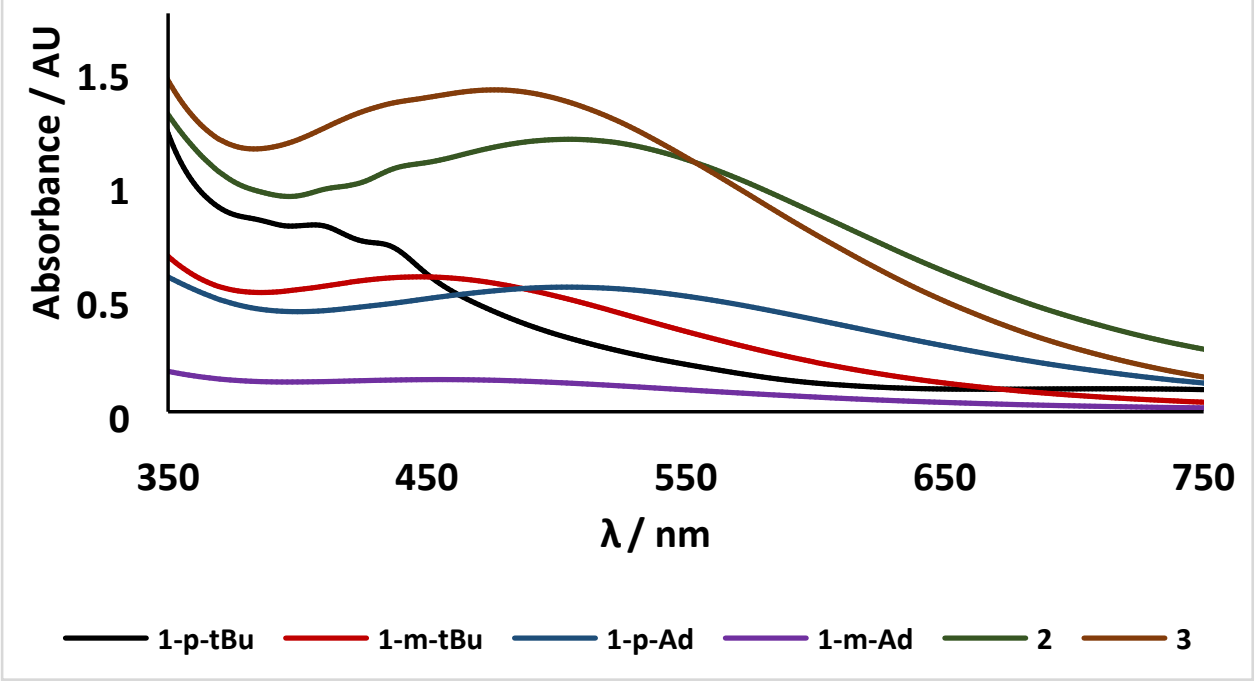

Table S2: $\lambda_{\max }$ values of selected di-Ce(IV) complexes.

\begin{tabular}{|c|c|}
\hline Compound & $\boldsymbol{\lambda}_{\max }$ \\
\hline & $\mathrm{nm}$ \\
\hline 1-p-tBu & 406.5 \\
\hline 1- $\boldsymbol{m}$ - ${ }^{\mathrm{t}} \mathrm{Bu}$ & 447.5 \\
\hline 1-p-Ad & 504 \\
\hline 1-m-Ad & 455 \\
\hline 2-p-tBu & 505 \\
\hline 3 & 476.5 \\
\hline
\end{tabular}




\section{S3 ROCOP Protocols}

\section{S3.1 Example procedure for $\mathrm{PA} / \mathrm{CHO}$ reactions, using $\left[\mathrm{NEt}_{4}\right]_{2}\left[\mathrm{Ce}_{2} \mathrm{Cl}_{2}\left(\mathrm{OSiMe}_{3}\right)_{4}\left(p \mathrm{TPt}^{\mathrm{t}}\right)\right]$ as catalyst.}

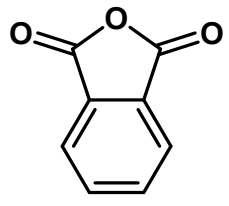

PA

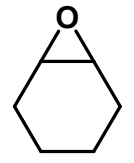

$\mathrm{CHO}$

\section{[cat]:[PA]:[CHO] = 1:100:800} neat, time, $100{ }^{\circ} \mathrm{C}$

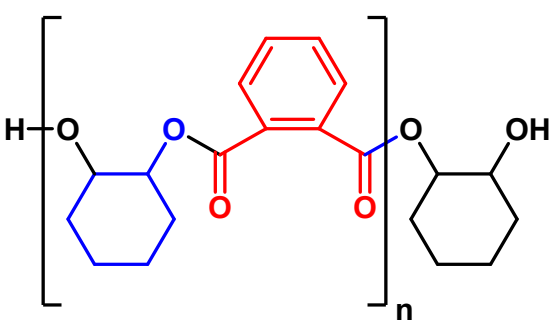

Equation S14: General reaction for anhydride/epoxide ROCOP.

In the glovebox a glass vial with Teflon-coated lid was charged with $\left[\mathrm{NEt}_{4}\right]_{2}\left[\mathrm{Ce}_{2} \mathrm{Cl}_{2}\left(\mathrm{OSiMe}_{3}\right)_{4}\left(p \mathrm{PPt}^{\mathrm{t}}\right)\right](14.2 \mathrm{mg}$, $0.0075 \mathrm{mmol}, 1$ equiv.) phthalic anhydride (111.1 mg, $0.75 \mathrm{mmol}, 100$ equiv.) and a stir bar. Cyclohexene oxide $(0.60 \mathrm{~mL}, 6.0 \mathrm{mmol}, 800$ equiv.) was added, the vial closed and then taken out of the glovebox. The reaction mixture was heated to $100{ }^{\circ} \mathrm{C}$ with constant stirring and after the appropriate reaction time the vial was opened to air and quenched by addition of $\mathrm{CDCl}_{3}(0.5 \mathrm{~mL})$. A sample of the crude polymer was analysed by ${ }^{1} \mathrm{H} N M R$ spectroscopy. The polymer was purified by evaporating volatiles under vacuum, dissolving the residue in a minimum volume of dichloromethane and precipitating the polymer by addition to stirring pentane.

SEC samples were prepared by filtering a solution ( $4 \mathrm{mg} \cdot \mathrm{mL}^{-1}$ in SEC grade THF) of the purified polymer.

For MALDI-ToF samples, the purified polymer, dithranol matrix and KI (cationizing agent) were dissolved in THF at $10 \mathrm{mg} \cdot \mathrm{mL}^{-1}$, and the solutions were mixed in a 2:2:1 volume ratio, respectively. A droplet ( $\left.2 \mu \mathrm{L}\right)$ of the resultant mixture was spotted on to the sample plate and submitted for MALDI-ToF MS analysis.

Diagnostic resonances of poly(cyclohexene phthalate): ${ }^{10}{ }^{1} \mathrm{H} N M R\left(500 \mathrm{MHz}, \mathrm{CDCl}_{3}\right) \delta_{\mathrm{H}} 7.59(\mathrm{br} \mathrm{m}, 2 \times \mathrm{Ar}-\mathrm{H}), 7.41$ (br m, $2 \times \mathrm{Ar}-\mathrm{H}), 5.15$ (br, $2 \times \mathrm{OC}-\mathrm{H}), 2.23\left(\mathrm{br} \mathrm{m},-\mathrm{CH}_{2}\right), 1.76\left(\mathrm{br} \mathrm{m},-\mathrm{CH}_{2}\right), 1.53\left(\mathrm{br} \mathrm{m},-\mathrm{CH}_{2}\right), 1.37\left(\mathrm{br} \mathrm{m},-\mathrm{CH}_{2}\right)$. ${ }^{13}\left\{{ }^{1} \mathrm{H}\right\}$ NMR $\left(500 \mathrm{MHz}, \mathrm{CDCl}_{3}\right) \delta_{\mathrm{c}} 166.8(\mathrm{O}-\mathrm{CH}-\mathrm{CH}-\mathrm{OC}=\mathrm{C}), 132.2(\operatorname{Ar} \underline{\mathrm{C}}), 131.2(\operatorname{Ar} \underline{\mathrm{C}}), 129.0(\operatorname{Ar} \underline{\mathrm{C}}), 74.8(\mathrm{O}-\underline{\mathrm{CH}}-\underline{\mathrm{C}} \mathrm{H}-$ $\mathrm{OC}=\mathrm{O}), 30.0\left(\mathrm{CH}_{2}-\mathrm{CH}_{2}-\mathrm{CH}-\mathrm{O}\right), 23.5\left(\mathrm{CH}_{2}-\mathrm{CH}_{2}-\mathrm{CH}-\mathrm{O}\right)$.

Figure S25: ${ }^{1 \mathrm{H}} \mathrm{NMR}$ spectrum of poly(cyclohexene phthalate) in $\mathrm{CDCl}_{3}$, produced by a $2.5 \mathrm{~h}$ reaction with catalyst 3.

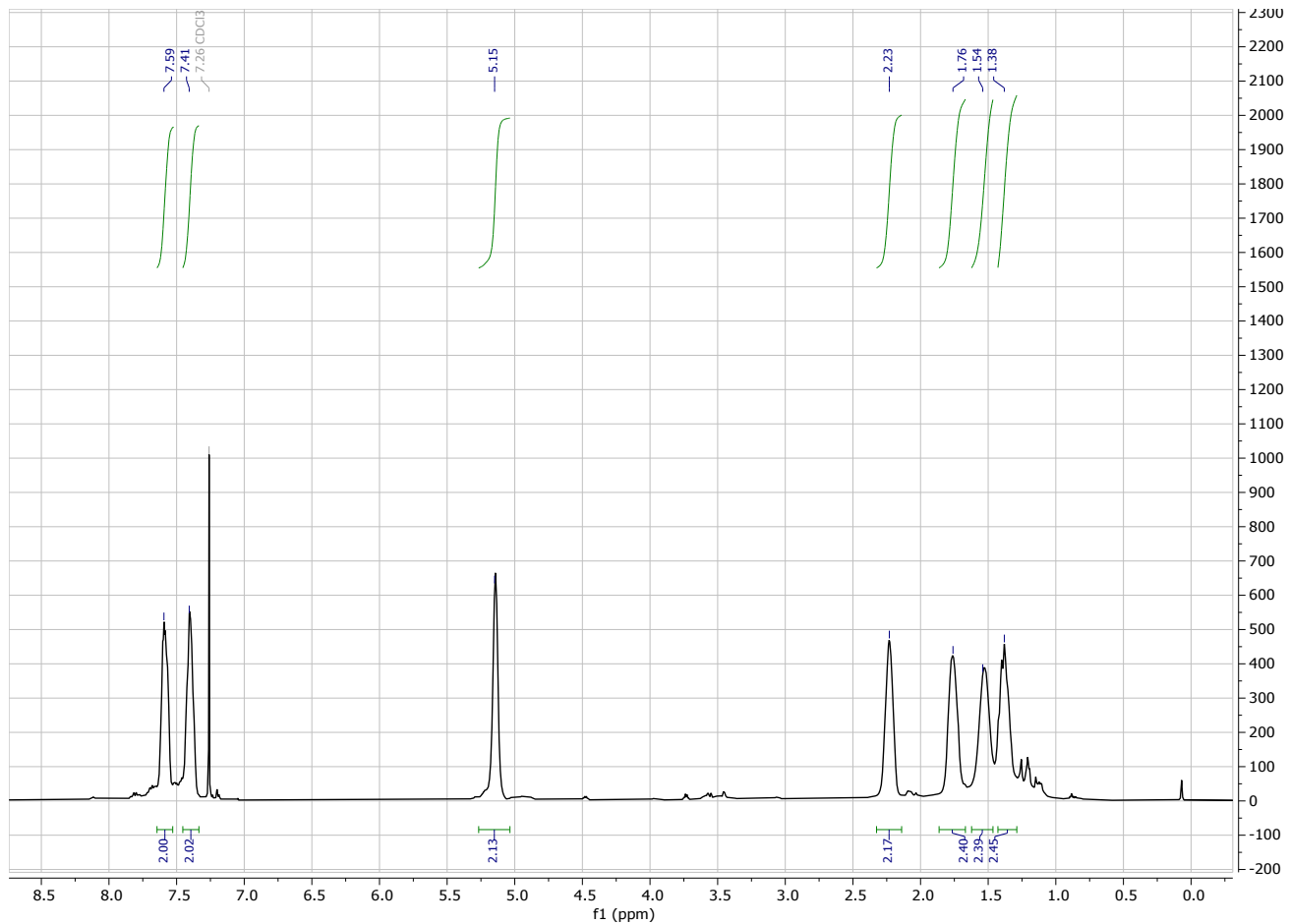


Figure S26: ${ }^{13} \mathrm{C}\left\{{ }^{1} \mathrm{H}\right\}$ NMR spectrum of poly (cyclohexene phthalate) in $\mathrm{CDCl}_{3}$, produced by a $2.5 \mathrm{~h}$ reaction with catalyst 3,100 equiv. PA and 800 equiv. $\mathrm{CHO}$.

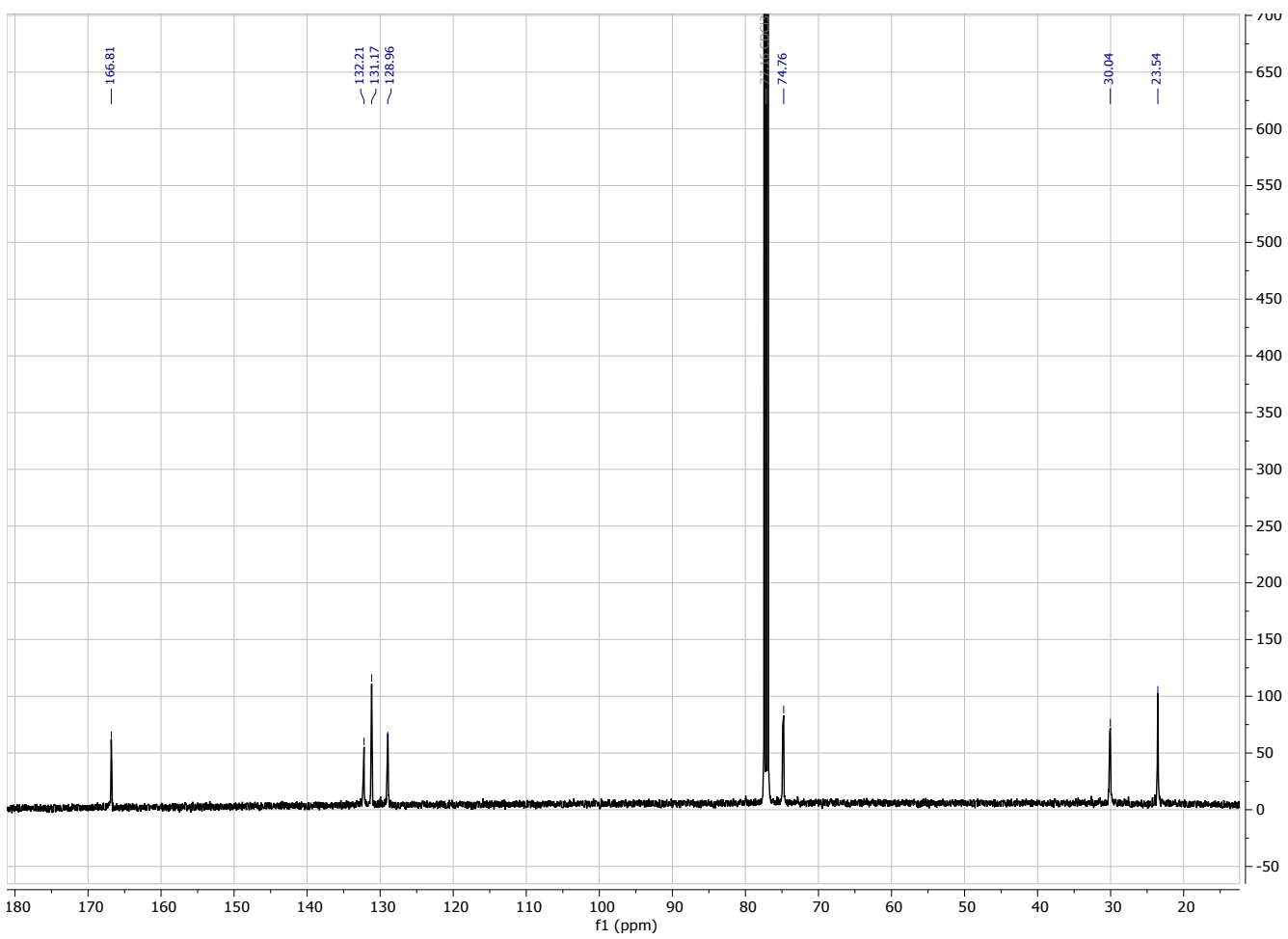

Figure S27: ${ }^{1} \mathrm{H}$ NMR spectrum of poly(cyclohexene phthalate) in $\mathrm{CDCl}_{3}$, produced by a $2 \mathrm{~h}$ reaction with catalyst 1-p-' $\mathrm{Bu}, 10$ equiv. PA and 80 equiv. $\mathrm{CHO}$. The small peak at $0.07 \mathrm{ppm}$ can be attributed to silicone grease present in the $\mathrm{CDCl}_{3}$ (confirmed in a spectrum of blank solvent). It is also observed in the spectrum in Figure $\mathrm{S} 7$ above, therefore is not due to a $-\mathrm{OSiMe}_{3}$ initiated polymer. Trace polyether, THF and hexane are also visible.

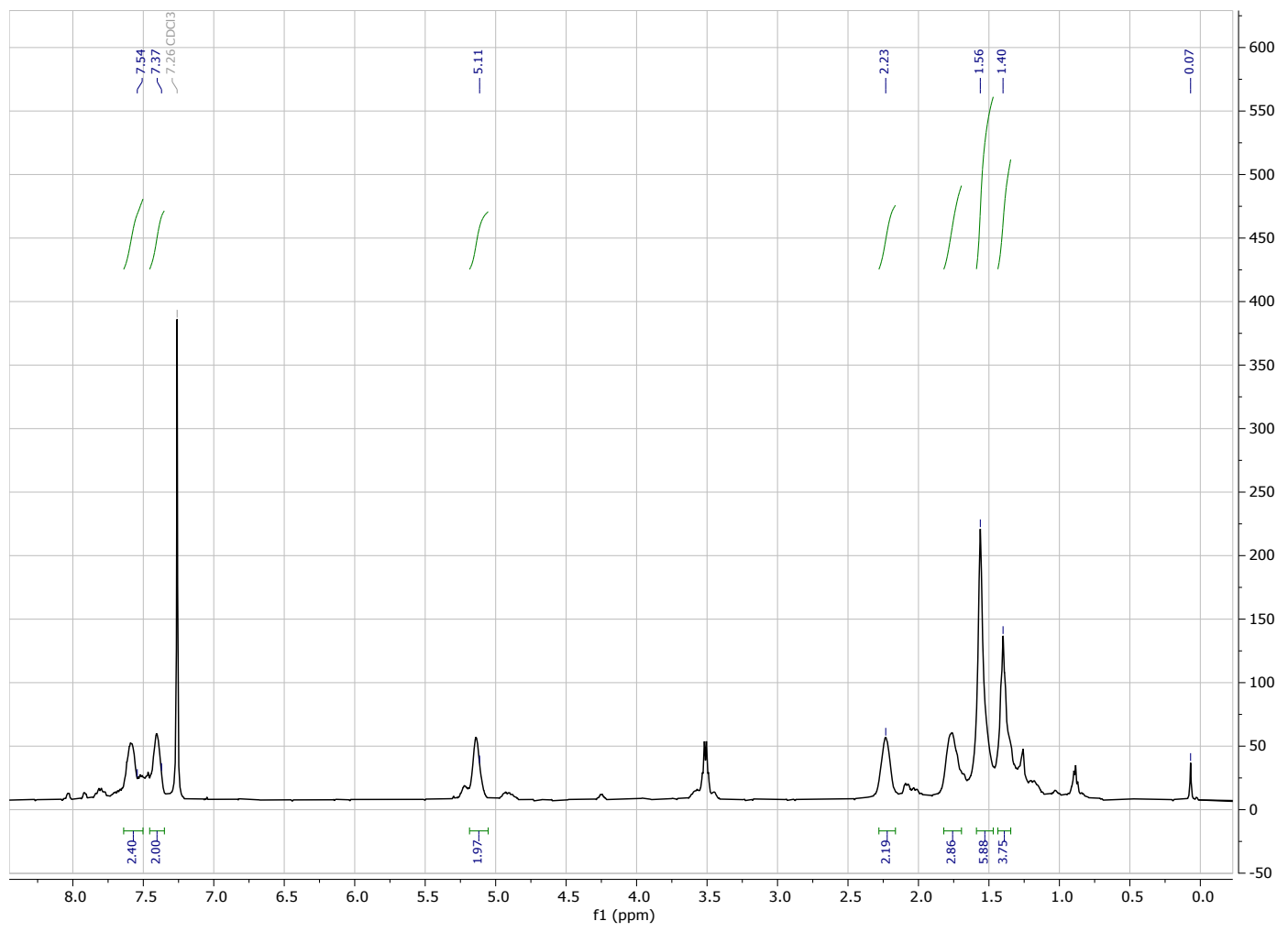


Table S3: Data for PA/CHO ROCOP at multiple time-points ${ }^{\mathrm{a}}$

\begin{tabular}{|c|c|c|c|c|c|c|c|c|}
\hline Entry & Catalyst & Loading & Time & $\begin{array}{c}\% \mathrm{PA} \\
\text { Conversion }^{\mathrm{b}}\end{array}$ & $\begin{array}{c}\% \text { Ester } \\
\text { Linkages }^{\mathrm{c}}\end{array}$ & TOF $^{\mathrm{e}}$ & $M_{n}(\exp )^{d}$ & $D^{d}$ \\
\hline & & mol\% & $\mathrm{h}$ & & & $\mathrm{mol} \cdot \mathrm{h}^{-1}$ & g. $\mathrm{mol}^{-1}$ & \\
\hline 1 & $1-m-{ }^{t} B u$ & 1.0 & 0.50 & 49 & 94 & 98 & 4690 & 1.29 \\
\hline 2 & $1-m-{ }^{t} B u$ & 1.0 & 0.75 & 86 & 97 & 115 & 5240 & 1.31 \\
\hline 3 & $1-m-{ }^{t} B u$ & 1.0 & 1.25 & 99 & 97 & 79 & 7120 & 1.32 \\
\hline \multirow{2}{*}{4} & \multirow{2}{*}{$1-p-{ }^{-} B u$} & \multirow{2}{*}{1.0} & \multirow{2}{*}{0.50} & \multirow{2}{*}{29} & \multirow{2}{*}{94} & \multirow{2}{*}{58} & $7730(5180)$ & $1.07(1.24)$ \\
\hline & & & & & & & 3420 & 1.05 \\
\hline 5 & $1-p-{ }^{t} B u$ & 1.0 & 1.25 & 83 & 96 & 66 & 6070 & 1.23 \\
\hline 6 & $1-p-{ }^{-} \mathrm{Bu}$ & 1.0 & 2.00 & 100 & 96 & 50 & 7360 & 1.22 \\
\hline 7 & 1-m-Ad & 1.0 & 0.17 & 38 & 91 & 229 & 2700 & 1.09 \\
\hline 8 & 1-m-Ad & 1.0 & 0.33 & 82 & 97 & 246 & 2800 & 1.08 \\
\hline 9 & $1-m-A d$ & 1.0 & 0.50 & 99 & 97 & 198 & 4400 & 1.10 \\
\hline 10 & 1-p-Ad & 1.0 & 0.50 & 51 & 97 & 102 & 3940 & 1.12 \\
\hline \multirow{2}{*}{11} & \multirow{2}{*}{ 1-p-Ad } & \multirow{2}{*}{1.0} & \multirow{2}{*}{0.75} & \multirow{2}{*}{84} & \multirow{2}{*}{97} & \multirow{2}{*}{112} & $10100(6200)$ & $1.06(1.27)$ \\
\hline & & & & & & & 4130 & 1.07 \\
\hline \multirow{2}{*}{12} & \multirow{2}{*}{ 1-p-Ad } & \multirow{2}{*}{1.0} & \multirow{2}{*}{1.25} & \multirow{2}{*}{99} & \multirow{2}{*}{99} & \multirow{2}{*}{79} & $10620(6730)$ & $1.07(1.28)$ \\
\hline & & & & & & & 4460 & 1.07 \\
\hline 13 & $2-p-{ }^{-} B u$ & 1.0 & 0.50 & 30 & 93 & 60 & 4420 & 1.05 \\
\hline \multirow{2}{*}{14} & \multirow{2}{*}{$2-p-{ }^{-} B u$} & \multirow{2}{*}{1.0} & \multirow{2}{*}{1.25} & \multirow{2}{*}{67} & \multirow{2}{*}{92} & \multirow{2}{*}{54} & $6620(4230)$ & $1.07(1.26)$ \\
\hline & & & & & & & 2580 & 1.09 \\
\hline & & & & & & & $9140(5700)$ & $1.05(1.24)$ \\
\hline 15 & 2-p-'Ви & 1.0 & 2.00 & 99 & 89 & 50 & 4090 & 1.07 \\
\hline 16 & 2 & 10 & 050 & 23 & 88 & 16 & $15920(7720)$ & $1.07(1.32)$ \\
\hline 10 & 3 & 1.0 & 0.50 & 23 & 80 & 40 & 4530 & 1.22 \\
\hline 17 & 3 & 10 & م00 & 85 & 95 & 43 & $16230(7840)$ & $1.08(1.35)$ \\
\hline 17 & 3 & 1.0 & 2.00 & כ0 & 93 & 45 & 5860 & 1.14 \\
\hline 18 & 3 & 10 & 250 & 09 & & & $17890(8360)$ & $1.07(1.38)$ \\
\hline 18 & 3 & 1.0 & 2.50 & 99 & 99 & 40 & 6490 & 1.14 \\
\hline 19 & 4 & 1.0 & 1.00 & 42 & 93 & 42 & - & - \\
\hline 20 & 4 & 10 & 200 & 97 & 04 & 19 & $10180(6530)$ & 1.05 (1.23) \\
\hline 20 & 4 & 1.0 & 2.00 & II & 94 & 45 & 4590 & 1.06 \\
\hline 21 & 5 & 1.0 & 1.00 & 27 & 61 & 27 & - & - \\
\hline 22 & 5 & 1.0 & 2.00 & 87 & 71 & 49 & 9400 & 1.38 \\
\hline 23 & {$\left[\mathrm{NEt}_{4}\right]_{2} \mathrm{CeCl}_{6}{ }^{\mathrm{f}}$} & 2.0 & 2.00 & 87 & 83 & 22 & 2700 & 1.23 \\
\hline
\end{tabular}

aStandard conditions: Reactions were run at $100{ }^{\circ} \mathrm{C}$ with a molar ratio of catalyst:PA:CHO of 1:100:800. ${ }^{b}$ Determined by ${ }^{1} \mathrm{H}$ NMR spectroscopy $\left(\mathrm{CDCl}_{3}\right)$ by integrating the normalized resonances for PA $(7.95 \mathrm{ppm})$ and

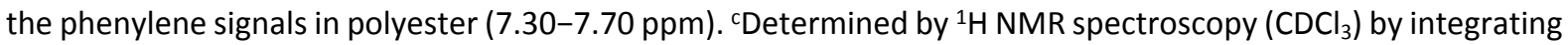
the normalized resonances for ester linkages $(5.10 \mathrm{ppm})$. ${ }^{d}$ Determined by SEC in THF using narrow- $\mathrm{M}_{\mathrm{n}}$ polystyrene standards to calibrate the instrument; $M_{n}$ multiplied by a correction factor of $1.85 .{ }^{11}$ Double entries represent bimodal distributions; the numbers in parentheses represent the total $M_{\mathrm{n}}$ and $\mathrm{B}$ values. ${ }^{\mathrm{T}}$ Turnover frequency (TOF) $=$ (number of moles of anhydride consumed/number of moles of catalyst)/time. ${ }^{\mathrm{f}} 2.0 \mathrm{~mol} \%$ catalyst loading. 
Table S4: Raw $M_{n}$ and $M_{w}$ data corresponding to runs in Table S3.

\begin{tabular}{|c|c|c|c|c|c|}
\hline Entry & Catalyst & $M_{w}(\text { raw, exp })^{a}$ & $M_{n}(\text { raw, exp })^{a}$ & $M_{n}(\text { corr, exp })^{b}$ & $D^{c}$ \\
\hline & & g.mol-1 & g.mol-1 & g.mol-1 & \\
\hline 1 & $1-m-{ }^{\mathrm{t} B u}$ & 3270 & 2540 & 4690 & 1.29 \\
\hline 2 & $1-m-{ }^{\mathrm{t}} \mathrm{Bu}$ & 3710 & 2830 & 5240 & 1.31 \\
\hline 3 & $1-m-{ }^{t} \mathrm{Bu}$ & 5090 & 3850 & 7120 & 1.32 \\
\hline \multirow{2}{*}{4} & \multirow{2}{*}{$1-p-{ }^{t} \mathrm{Bu}$} & 4465 (3480) & $4180(2800)$ & $7730(5180)$ & $1.07(1.24)$ \\
\hline & & 1950 & 1850 & 3420 & 1.05 \\
\hline 5 & $1-p-{ }^{t} \mathrm{Bu}$ & 4050 & 3280 & 6070 & 1.23 \\
\hline 6 & $1-p-{ }^{t} \mathrm{Bu}$ & 4870 & 3980 & 7360 & 1.22 \\
\hline 7 & 1-m-Ad & 1600 & 1460 & 2700 & 1.09 \\
\hline 8 & 1-m-Ad & 1630 & 1510 & 2800 & 1.08 \\
\hline 9 & $1-m-A d$ & 2620 & 2380 & 4400 & 1.10 \\
\hline 10 & $1-p-A d$ & 2380 & 2130 & 3940 & 1.12 \\
\hline \multirow{2}{*}{11} & \multirow{2}{*}{$1-p-A d$} & $5770(4260)$ & $5460(3350)$ & $10100(6200)$ & $1.06(1.27)$ \\
\hline & & 2440 & 2230 & 4130 & 1.07 \\
\hline \multirow{2}{*}{12} & \multirow{2}{*}{$1-p-A d$} & $6120(4650)$ & $5740(3640)$ & $10620(6730)$ & $1.07(1.28)$ \\
\hline & & 2590 & 2410 & 4460 & 1.07 \\
\hline 13 & $2-p-{ }^{t} \mathrm{Bu}$ & 2500 & 2390 & 4420 & 1.05 \\
\hline \multirow{2}{*}{14} & \multirow{2}{*}{$2-p-{ }^{t} \mathrm{Bu}$} & $3820(2890)$ & $3580(2290)$ & $6620(4230)$ & $1.07(1.26)$ \\
\hline & & 1520 & 1400 & 2580 & 1.09 \\
\hline \multirow{2}{*}{15} & \multirow{2}{*}{$2-p-{ }^{t} B u$} & 5190 (3800) & 4940 (3080) & $9140(5700)$ & $1.05(1.24)$ \\
\hline & & 2362 & 2210 & 4090 & 1.7 \\
\hline \multirow{2}{*}{16} & \multirow{2}{*}{3} & $9210(5500)$ & 8610 (4170) & $15920(7720)$ & $1.07(1.32)$ \\
\hline & & 3000 & 2450 & 4530 & 1.22 \\
\hline \multirow{2}{*}{17} & \multirow{2}{*}{3} & 9500 (5720) & 8770 (4240) & $16230(7840)$ & $1.08(1.35)$ \\
\hline & & 3600 & 3170 & 5860 & 1.14 \\
\hline \multirow{2}{*}{18} & \multirow{2}{*}{3} & $10310(6230)$ & $9670(4520)$ & $17890(8360)$ & $1.07(1.38)$ \\
\hline & & 4000 & 3510 & 6490 & 1.14 \\
\hline 19 & 4 & - & - & - & - \\
\hline \multirow{2}{*}{20} & \multirow{2}{*}{4} & $5780(4330)$ & $5500(3530)$ & $10180(6530)$ & $1.05(1.23)$ \\
\hline & & 2640 & 2480 & 4590 & 1.06 \\
\hline 21 & 5 & - & - & - & - \\
\hline 22 & 5 & 7020 & 5080 & 9400 & 1.38 \\
\hline 23 & {$\left[\mathrm{NEt}_{4}\right]_{2} \mathrm{CeCl}_{6}{ }^{\mathrm{f}}$} & 1790 & 1460 & 2700 & 1.23 \\
\hline
\end{tabular}

${ }^{a}$ Double entries represent bimodal distributions; the numbers in parentheses represent the total $M_{\mathrm{w}}, M_{\mathrm{n}}$ and $\mathrm{\theta}$ values. ${ }^{b} M_{\mathrm{n}}$ multiplied by a correction factor of 1.85 . ${ }^{\mathrm{C}} \mathrm{B}=M_{\mathrm{w}} / M_{\mathrm{n}}$. For total molar masses, the entire peak and shoulders were integrated; for bimodal samples the minimum between the two peaks was chosen as the boundary between the two integrated areas. 


\section{S3.2 Example procedure for ROCOP substrate scope}

In the glovebox a glass vial with Teflon-coated lid was charged with 1- $\mathbf{m - A d ~ ( 1 8 . 1 ~ m g , ~} 0.0075$ mmol, 1 equiv.) and the relevant anhydride $(0.75 \mathrm{mmol}, 100$ equiv. $)$, and a stir bar.

For CHO and LO reactions, the relevant epoxide $(6.0 \mathrm{mmol}, 800$ equiv.) was added, the vial closed and then taken out of the glovebox.

For $P O$ reactions, propylene oxide $(0.42 \mathrm{~mL}, 6.0 \mathrm{mmol}, 800$ equiv.) and toluene $(1.5 \mathrm{~mL})$ were added the vial closed and then taken out of the glovebox.

The reaction mixture was heated to $100{ }^{\circ} \mathrm{C}$ with constant stirring. After the appropriate reaction time the vial was opened to air and the contents rinsed into a round-bottom flask with dichloromethane, volatiles were removed under vacuum and the crude polymer analysed by ${ }^{1} \mathrm{H} \mathrm{NMR}$ spectroscopy $\left(\mathrm{CDCl}_{3}\right)$. The polymer was purified by dissolving in a minimum volume of dichloromethane and precipitating by addition to stirring pentane.

SEC/MALDI-TOF sample preparations are identical as for the $\mathrm{PA} / \mathrm{CHO}$ reactions.

Diagnostic resonances of poly(propylene phthalate): ${ }^{12}{ }^{1} \mathrm{H}$ NMR $\left(600 \mathrm{MHz}, \mathrm{CDCl}_{3}\right) \delta_{\mathrm{H}} 7.70(\mathrm{br} \mathrm{m}, 2 \mathrm{H}, \mathrm{Ar}-\mathrm{H}), 7.48$ (br m, $2 \mathrm{H}, \mathrm{Ar}-\mathrm{H}), 5.41\left(\mathrm{br}, 1 \mathrm{H},(\mathrm{CO}) \mathrm{O}-\mathrm{CH}\left(\mathrm{CH}_{3}\right)-\mathrm{CH}_{2}\right), 4.40$ (br m, 2H, (CO)O-CH(CH$\left.\left.)_{3}\right)-\mathrm{CH}_{2}\right), 1.35$ (br, 3H, (CO)O$\mathrm{CH}\left(\mathrm{CH}_{3}\right) \mathrm{CH}_{2}$ ). (Residual pentane visible in spectrum below.)

Figure S28: ${ }^{1} \mathrm{H}$ NMR spectrum of poly(propylene phthalate) in $\mathrm{CDCl}_{3}$, produced by a $2.5 \mathrm{~h}$ reaction with catalyst 1-m-Ad, 100 equiv. PA and 800 equiv. PO.

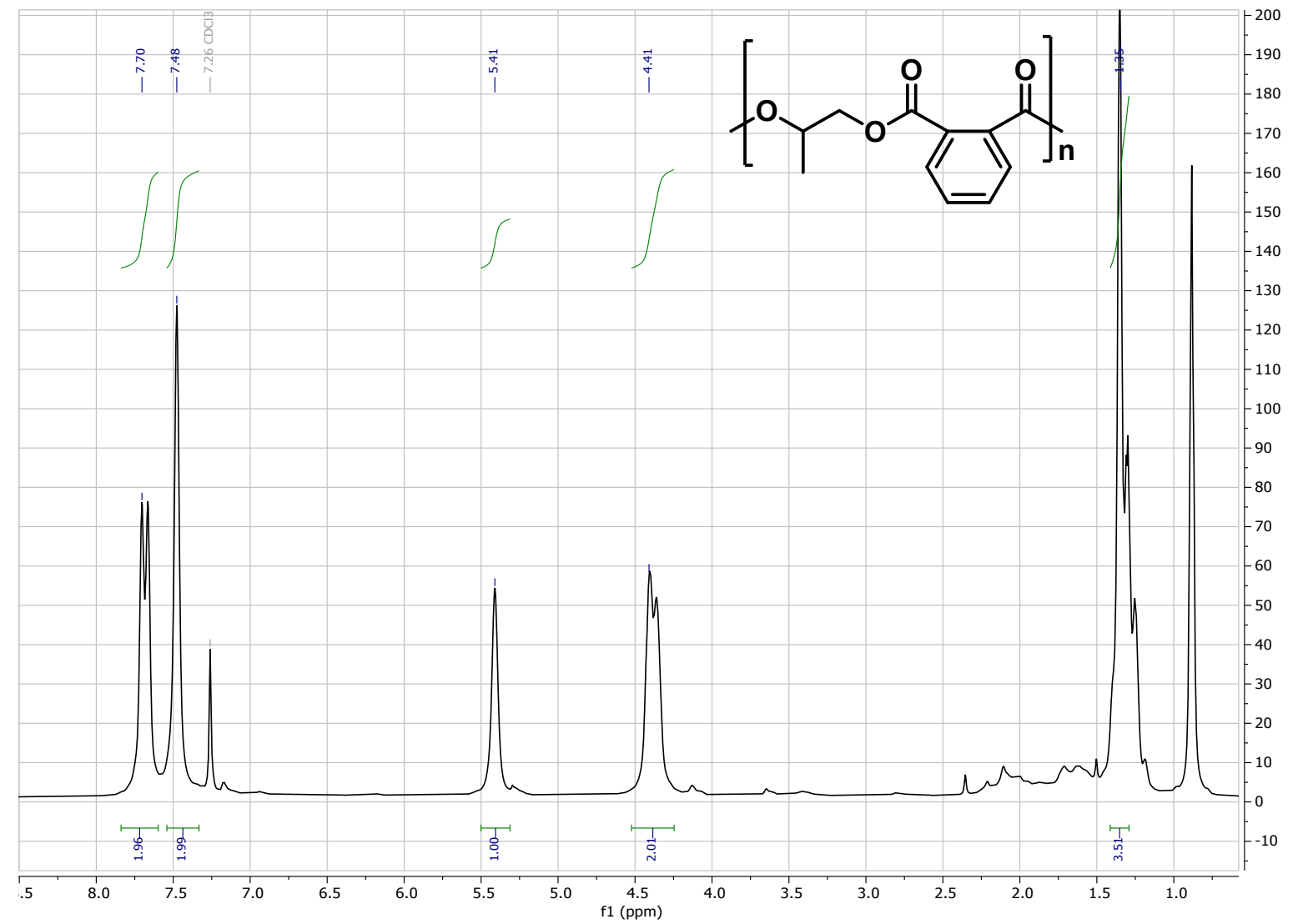


Diagnostic resonances of poly(propylene succinate): ${ }^{13}{ }^{1} \mathrm{H} ~ N M R\left(500 \mathrm{MHz}, \mathrm{CDCl}_{3}\right) \delta_{\mathrm{H}} 5.12\left(\mathrm{br}, 1 \mathrm{H},(\mathrm{CO}) \mathrm{O}-\mathrm{CH}\left(\mathrm{CH}_{3}\right)-\right.$ $\mathrm{CH} 2), 4.17\left(\mathrm{~m}, 1 \mathrm{H},(\mathrm{CO}) \mathrm{O}-\mathrm{CH}\left(\mathrm{CH}_{3}\right)-\mathrm{CH}_{2}\right), 4.07\left(\mathrm{~m}, 1 \mathrm{H},(\mathrm{CO}) \mathrm{O}-\mathrm{CH}\left(\mathrm{CH}_{3}\right)-\mathrm{CH}_{2}\right), 2.61(\mathrm{~m}, 4 \mathrm{H},(\mathrm{CO})-\mathrm{CH} 2-\mathrm{CH} 2-(\mathrm{CO})), 1.22$ $\left(\mathrm{d}, 4 \mathrm{H},(\mathrm{CO}) \mathrm{O}-\mathrm{CH}\left(\mathrm{CH}_{3}\right)-\mathrm{CH}_{2}\right)$.

Figure S29: ${ }^{1} \mathrm{H}$ NMR spectrum of poly(propylene succinate) in $\mathrm{CDCl}_{3}$, produced by a $2.5 \mathrm{~h}$ reaction with catalyst 1-m-Ad, 100 equiv. SA and 800 equiv. PO.

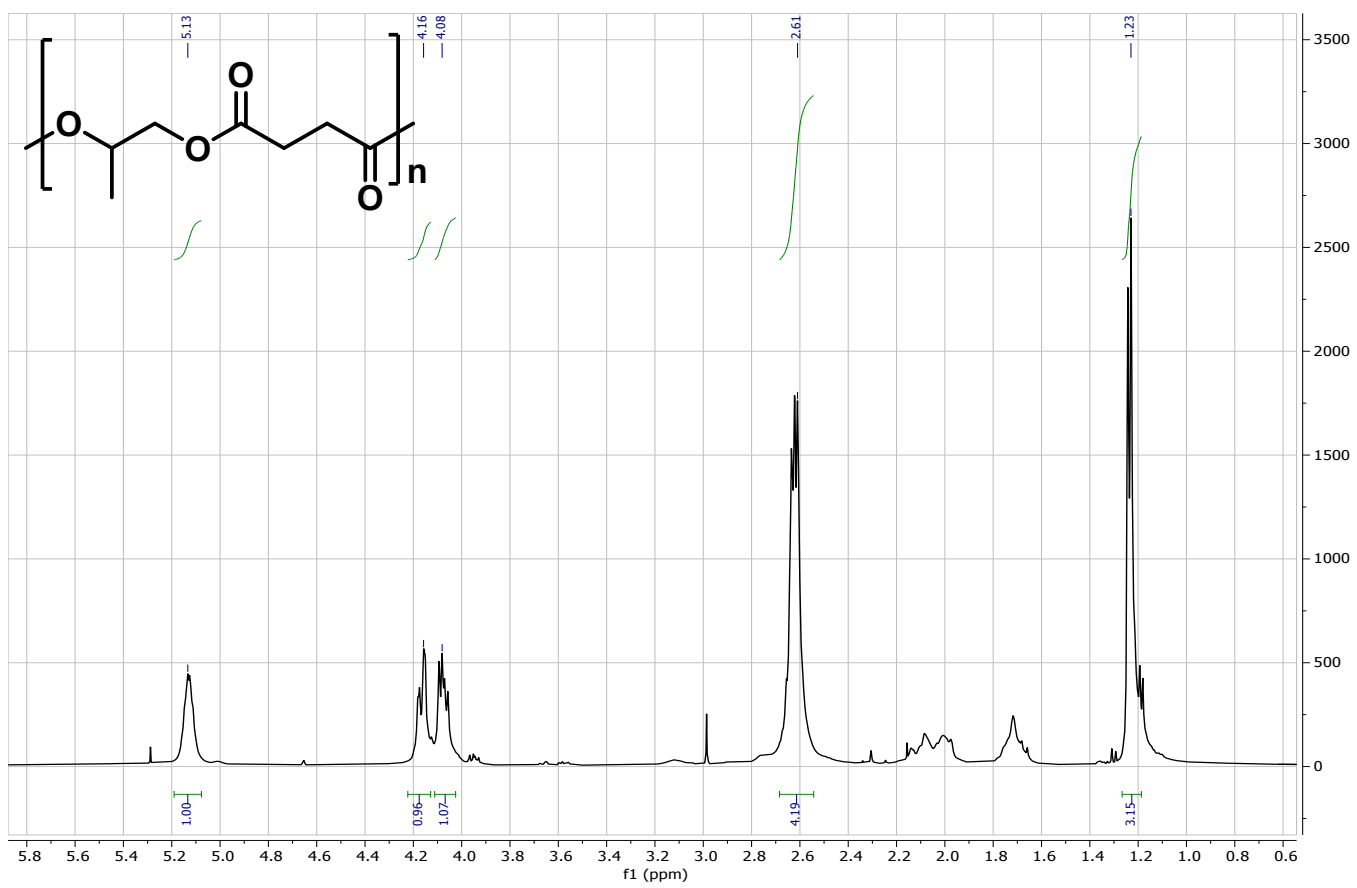

Diagnostic resonances of poly(cyclohexene succinate): ${ }^{14}{ }^{1} \mathrm{H} \mathrm{NMR}\left(500 \mathrm{MHz}, \mathrm{CDCl}_{3}\right) \delta_{\mathrm{H}} 4.80(\mathrm{br}, 2 \times \mathrm{OC}-\mathrm{H}), 2.54$ (br, $\left.4 \mathrm{H},(\mathrm{CO})-\mathrm{CH}_{2}-\mathrm{CH}_{2}-(\mathrm{CO})\right), 2.01$ (br, $4 \mathrm{H}$, cyclohexyl $\left.\mathrm{CH}_{2}\right), 1.70\left(\mathrm{br}, 4 \mathrm{H}\right.$, cyclohexyl $\left.\mathrm{CH}_{2}\right)$.

Figure S30: ${ }^{1} \mathrm{H}$ NMR spectrum of poly(cyclohexene succinate) in $\mathrm{CDCl}_{3}$, produced by a $0.5 \mathrm{~h}$ reaction with catalyst 1-m-Ad, 100 equiv. SA and 800 equiv. $\mathrm{CHO}$.

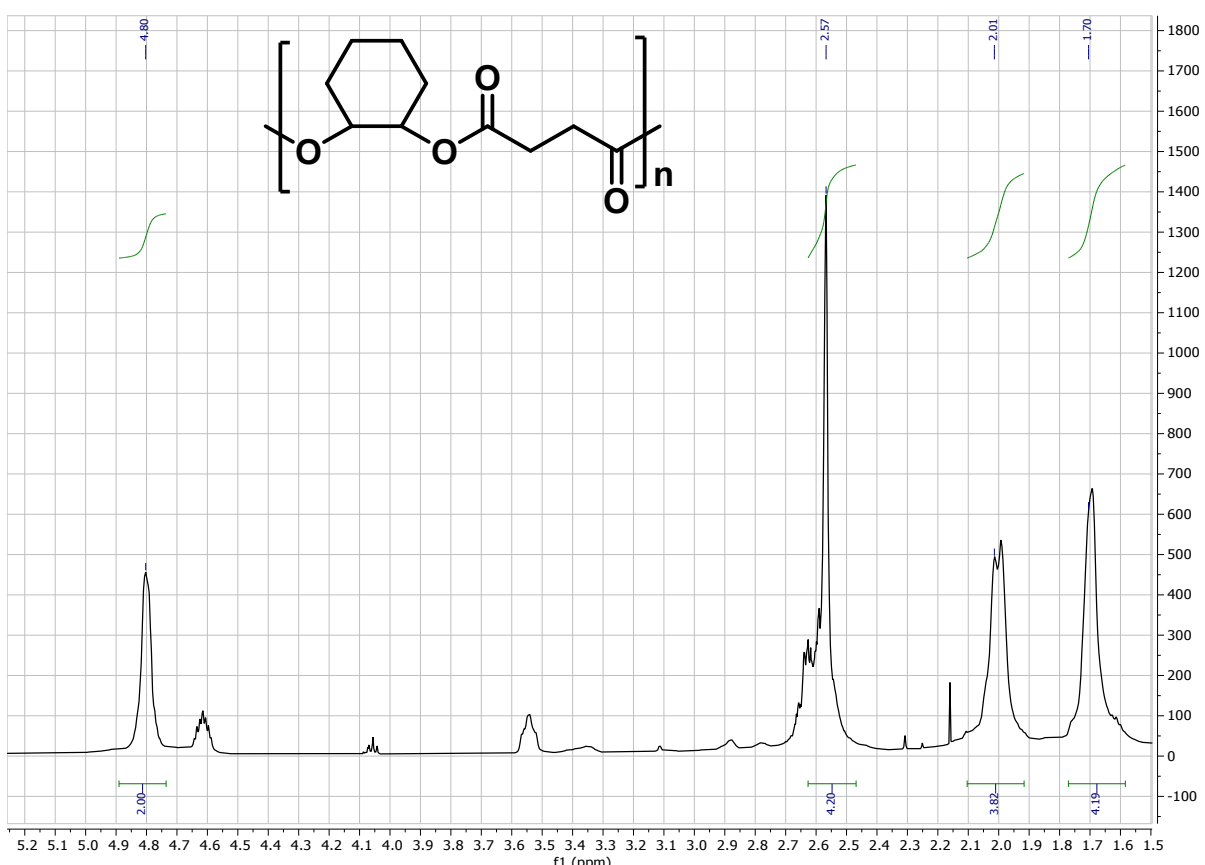


Diagnostic resonances of poly(limonene phthalate): ${ }^{15}{ }^{1} \mathrm{H} \mathrm{NMR}\left(500 \mathrm{MHz}, \mathrm{CDCl}_{3}\right)$ $\delta_{\mathrm{H}}$ 7.64-7.30 (br m, 4H, ArH), $5.41(\mathrm{br}, 1 \mathrm{H}, \mathrm{OCCH}), 4.55\left(\mathrm{~m}, 2 \mathrm{H}, \mathrm{C}=\mathrm{CH}_{2}\right)$.

Figure S31: ${ }^{1} \mathrm{H}$ NMR spectrum of poly(limonene phthalate) in $\mathrm{CDCl}_{3}$, produced by a $18 \mathrm{~h}$ reaction with catalyst 1-m-Ad, 100 equiv. PA and 800 equiv. LO.

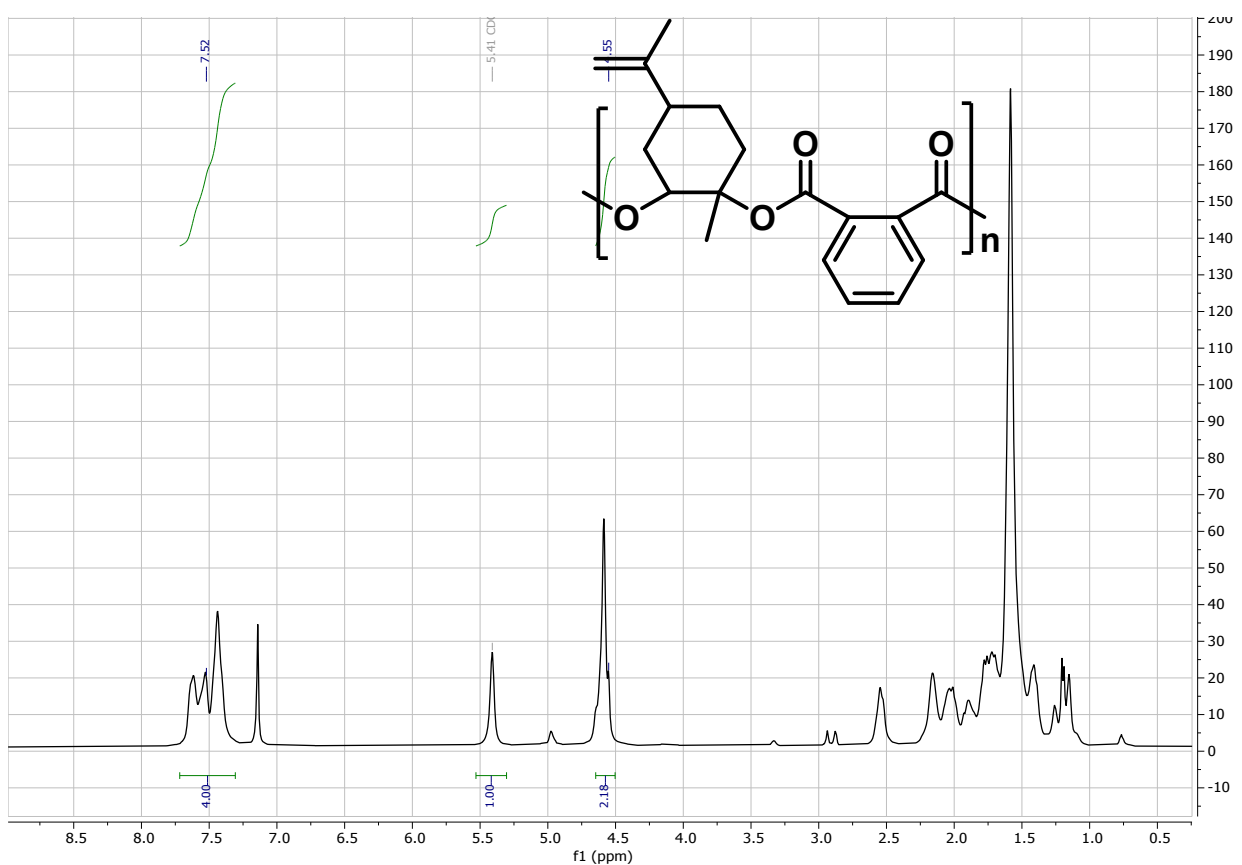

Diagnostic resonances of poly(limonene succinate): ${ }^{16}{ }^{1} \mathrm{H} \mathrm{NMR}\left(500 \mathrm{MHz}, \mathrm{CDCl}_{3}\right) \delta_{\mathrm{H}} 5.20(\mathrm{br}, 1 \mathrm{H}, \mathrm{OCCH}), 4.82(\mathrm{~m}$, $\left.2 \mathrm{H}, \mathrm{C}=\mathrm{CH}_{2}\right), 2.65\left(\mathrm{br}, 4 \mathrm{H},(\mathrm{CO})-\mathrm{CH}_{2}-\mathrm{CH}_{2}-(\mathrm{CO})\right)$.

Figure S32: ${ }^{1} \mathrm{H}$ NMR spectrum of poly(limonene succinate) in $\mathrm{CDCl}_{3}$, produced by a $18 \mathrm{~h}$ reaction with catalyst 1$m$-Ad, 100 equiv. SA and 800 equiv. LO.

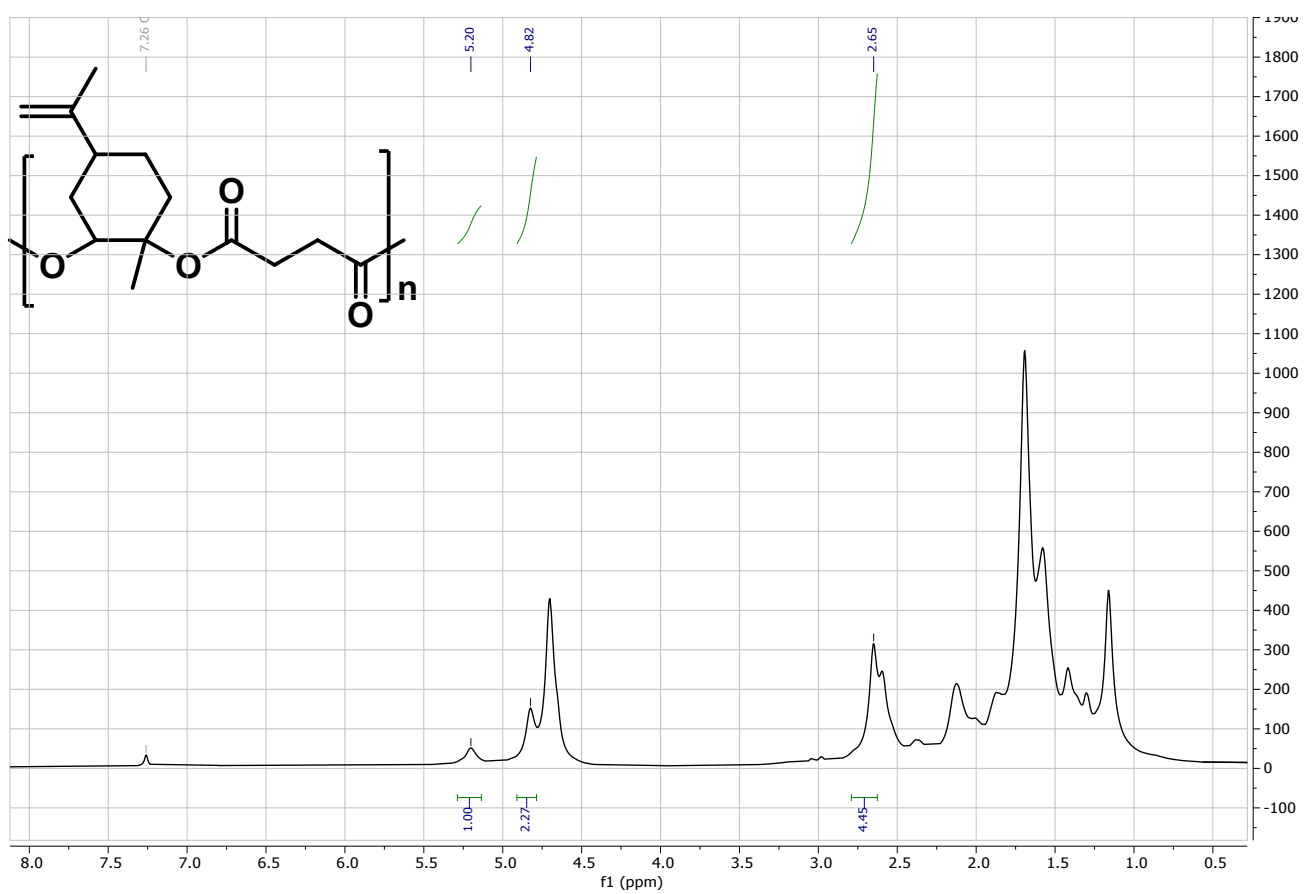


Table S5: Substrate scope using 1-m-Ad as catalyst. ${ }^{a}$

\begin{tabular}{|c|c|c|c|c|c|c|c|c|}
\hline $\begin{array}{l}\text { Entry / } \\
\text { catalyst } \\
\text { loading }\end{array}$ & $\begin{array}{l}\text { Monomer } \\
\text { Combination }\end{array}$ & Solvent & TON $^{b}$ & TOF & $\begin{array}{c}\% \\
\text { Ester }\end{array}$ & $\begin{array}{c}M_{w} \\
(\exp )^{e}\end{array}$ & $M_{n}(\exp )^{e}$ & $D^{e}$ \\
\hline & & & & $\mathrm{mol} \cdot \mathrm{h}^{-1}$ & & g. $\mathrm{mol}^{-1}$ & g. mol-1 & \\
\hline \multirow[t]{2}{*}{1 / 1 mol\% } & \multirow[t]{2}{*}{ PO/PA } & \multirow[t]{2}{*}{ toluene } & \multirow[t]{2}{*}{100} & \multirow[t]{2}{*}{40} & \multirow[t]{2}{*}{96} & $\begin{array}{c}5330 \\
(5520)\end{array}$ & $\begin{array}{c}4990 \\
(3890)\end{array}$ & $\begin{array}{c}1.07 \\
(1.42)\end{array}$ \\
\hline & & & & & & 1790 & 1640 & 1.09 \\
\hline \multirow[t]{2}{*}{$2^{c} / 0.5 \mathrm{~mol} \%$} & \multirow[t]{2}{*}{ PO/PA } & \multirow[t]{2}{*}{ toluene } & \multirow[t]{2}{*}{99} & \multirow[t]{2}{*}{11} & \multirow[t]{2}{*}{97} & $\begin{array}{c}23853 \\
(20660)\end{array}$ & $\begin{array}{c}19370 \\
(13510)\end{array}$ & $\begin{array}{c}1.23 \\
(1.53)\end{array}$ \\
\hline & & & & & & 6290 & 5750 & 1.09 \\
\hline \multirow[t]{2}{*}{$3^{c} / 1$ mol\% } & \multirow[t]{2}{*}{ LO/PA } & \multirow[t]{2}{*}{ neat } & \multirow[t]{2}{*}{82} & \multirow[t]{2}{*}{5} & \multirow[t]{2}{*}{94} & $\begin{array}{c}8490 \\
(6370)\end{array}$ & $\begin{array}{c}7740 \\
(4740)\end{array}$ & $\begin{array}{c}1.10 \\
(1.34)\end{array}$ \\
\hline & & & & & & 3280 & 3020 & 1.09 \\
\hline $4^{d} / 1$ mol\% & $\mathrm{CHO} / \mathrm{SA}$ & neat & 92 & 184 & 90 & 4070 & 2790 & 1.46 \\
\hline 5c/ $0.5 \mathrm{~mol} \%$ & $\mathrm{CHO} / \mathrm{SA}$ & neat & 98 & 11 & 88 & 5200 & 3860 & 1.35 \\
\hline \multirow[t]{2}{*}{6 / 1 mol\% } & \multirow[t]{2}{*}{$\mathrm{PO} / \mathrm{SA}$} & \multirow[t]{2}{*}{ toluene } & \multirow[t]{2}{*}{98} & \multirow[t]{2}{*}{39} & \multirow[t]{2}{*}{92} & $\begin{array}{c}5320 \\
(1560)\end{array}$ & $\begin{array}{c}3890 \\
(1220)\end{array}$ & $\begin{array}{c}1.37 \\
(1.28)\end{array}$ \\
\hline & & & & & & 1090 & 1070 & 1.02 \\
\hline \multirow[t]{2}{*}{ 7c/ 1 mol\% } & \multirow[t]{2}{*}{ LO/SA } & \multirow[t]{2}{*}{ neat } & \multirow[t]{2}{*}{89} & \multirow[t]{2}{*}{5} & \multirow[t]{2}{*}{86} & $\begin{array}{c}1720 \\
(1890)\end{array}$ & $\begin{array}{c}1540 \\
(1250)\end{array}$ & $\begin{array}{c}1.11 \\
(1.51)\end{array}$ \\
\hline & & & & & & 768 & 760 & 1.01 \\
\hline
\end{tabular}

aStandard conditions: Reactions were run at $100{ }^{\circ} \mathrm{C}$ for $2.5 \mathrm{~h}$ with a molar ratio of 1-m-Ad:anhydride:epoxide of 1:100:800. Due to the low boiling point of $P O$ toluene was used as solvent in the indicated reactions. ${ }^{b}$ Determined by ${ }^{1} \mathrm{H}$ NMR spectroscopy $\left(\mathrm{CDCl}_{3}\right)$ by integrating the normalized resonances for anhydride and polyester (conversion); and for ester and ether linkages (selectivity). ${ }^{c} 18 \mathrm{~h}$ reaction time. ${ }^{\mathrm{d}} 0.5 \mathrm{~h}$ reaction time. eNo correction factor has been applied for $M_{n}$. Double entries represent bimodal distributions, the numbers in parentheses represent the total $M_{\mathrm{n}}$ and $\theta$ values. $\theta=M_{\mathrm{w}} / M_{\mathrm{n}}$. ${ }^{\text {fBased }}$ on two polymer chains formed per catalyst molecule, where $M_{n}$ (calc) = (molar mass of repeat unit $\mathrm{x} \%$ anhydride conversion)/(catalyst loading $\mathrm{x} 2$ ). To the best of our knowledge, no correction factors have been reported for these monomer combinations which limits the comparison of the observed and calculated $M_{n}$ values for these monomer combinations. 


\section{S3.3 Rate equation determination experiments}

In the glovebox a $10 \mathrm{~cm}^{3}$ ampoule was charged with 1-p- ${ }^{\mathrm{t}} \mathrm{Bu}(56.6 \mathrm{mg}, 0.03 \mathrm{mmol}, 1$ equiv.) and phthalic anhydride ( $444.3 \mathrm{mg}, 3.0 \mathrm{mmol}, 100$ equiv.), and a stir bar. Cyclohexene oxide ( $2.4 \mathrm{~mL}, 24 \mathrm{mmol}, 800$ equiv.) was added and the reaction mixture was then heated to $100{ }^{\circ} \mathrm{C}$ with constant stirring. At the start of the reaction and every 15 minutes thereafter, a few drops of the reaction mixture were transferred via cannula, and any reaction halted, by addition to a NMR tube containing $0.5 \mathrm{~mL} \mathrm{CDCl}{ }_{3}$ at $0{ }^{\circ} \mathrm{C}$. The ${ }^{1} \mathrm{H} N M R$ spectrum of each sample was recorded, allowing monitoring of PA conversion and \% ester linkages over time. [1-p-tBu] was varied by changing the mass of $\mathbf{1}-\boldsymbol{p}$ - ${ }^{\mathrm{t}} \mathrm{Bu}$ and keeping all other variables constant. [CHO] was altered by decreasing the volume used from the standard 800 equiv. $\mathrm{CHO}$ and replacing the lost volume with the same volume of toluene. [PA] was calculated from the ${ }^{1} \mathrm{H}$ NMR spectra.
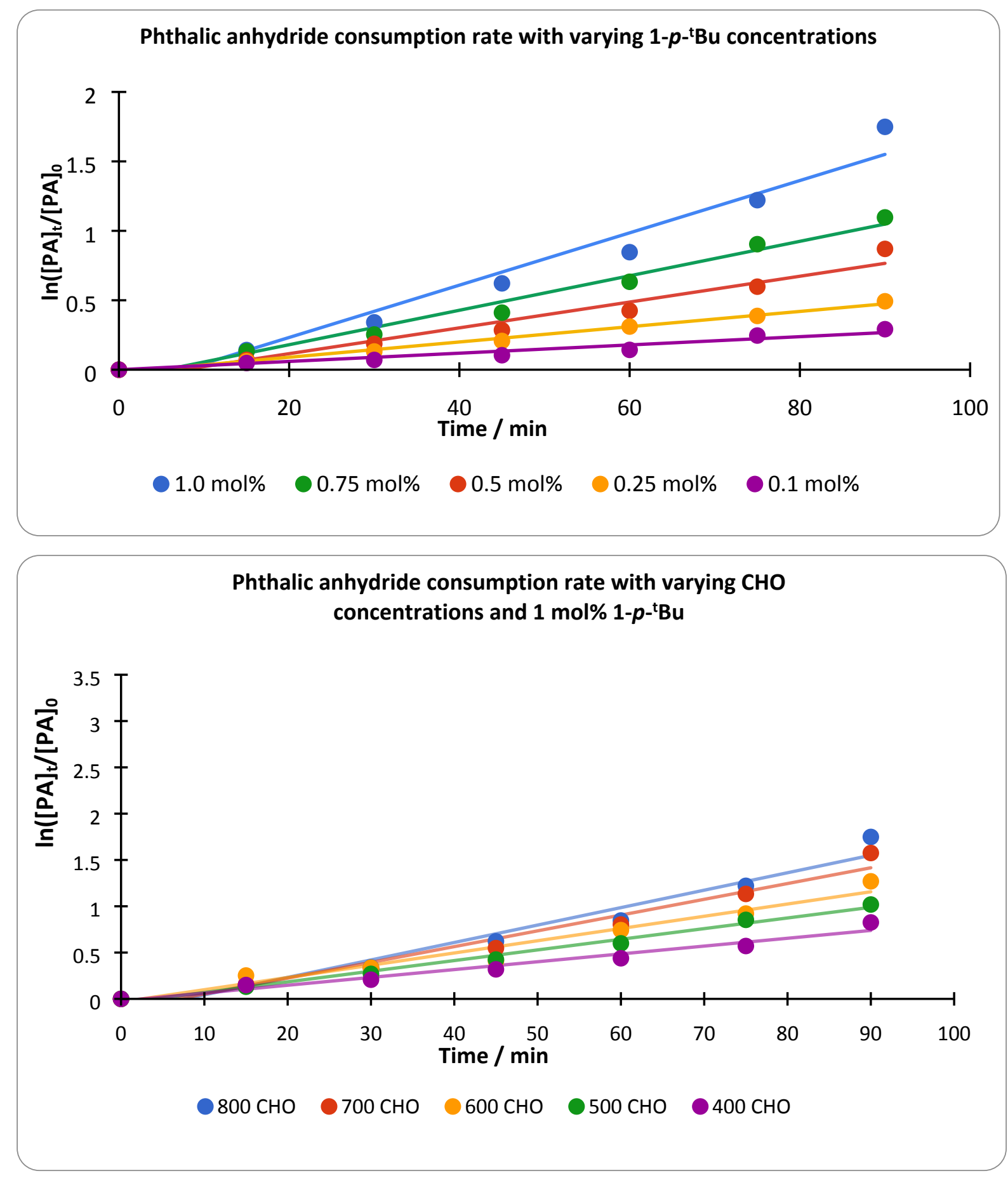


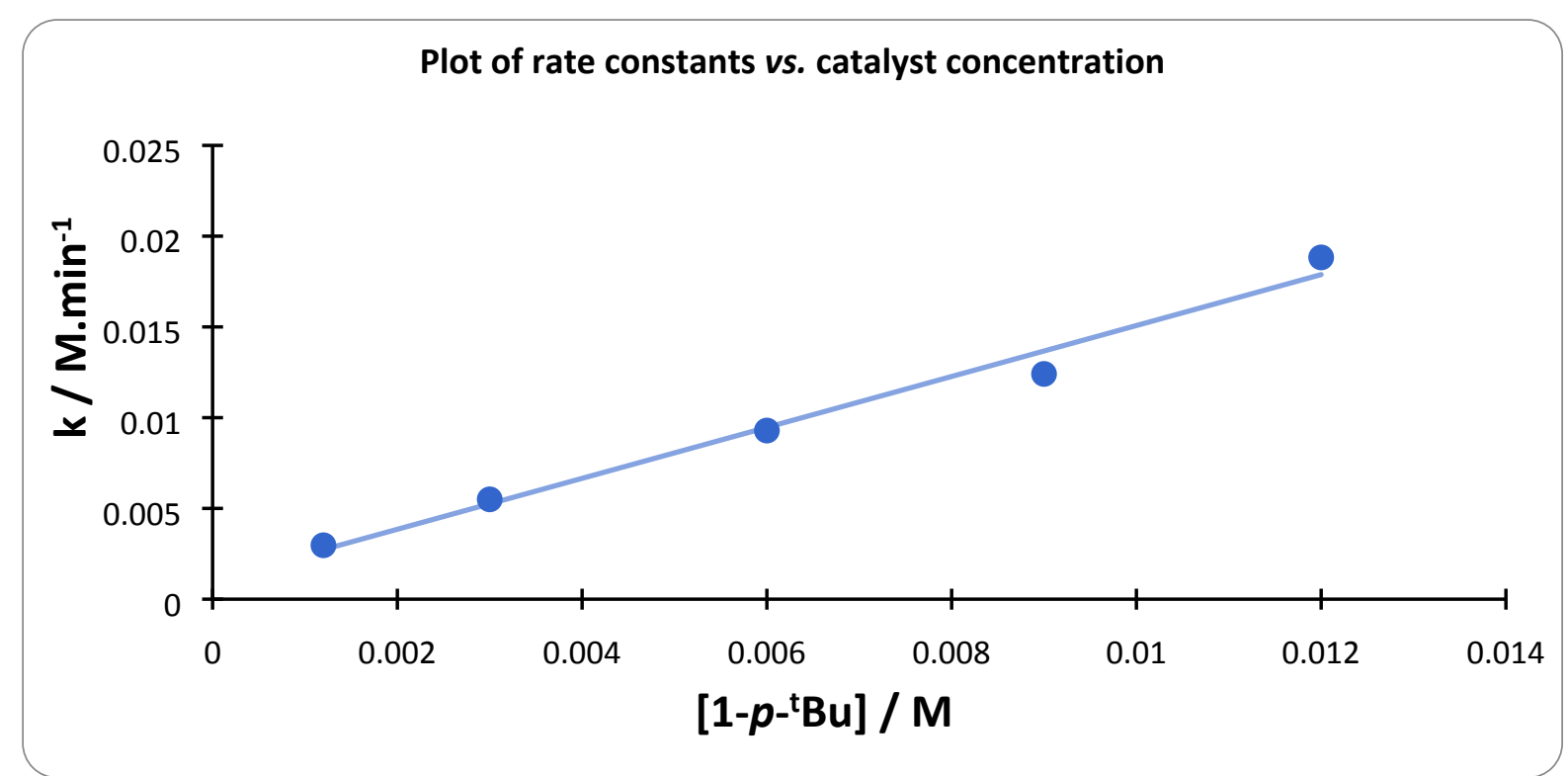

Figure S33: Kinetic plots. Top: Plot showing how the rate of PA consumption changes with different loadings of 1-p-tBu. Each line represents a different \% catalyst loading (i.e., concentration), and it is clear that the steeper lines for higher catalyst concentrations represent faster PA consumption. Middle: Plot showing how the rate of PA consumption changes with different concentrations of $\mathrm{CHO}$, but a constant concentration of 1-p-t $\mathrm{Bu}$. Each line represents a different $\mathrm{CHO}$ concentration, written in terms of $\mathrm{CHO}$ equivalents here for simplicity. We observe higher rates for higher $\mathrm{CHO}$ concentrations. Bottom: Plot of the rate constants $(k)$ determined at each value of [1-p-t $\mathrm{Bu}$ ] against the values of [1-p-t $\mathrm{Bu}$ ]. The data fit a straight line of best fit, suggesting that the overall polymerisation reaction has a first order dependence on catalyst concentration.

\section{S3.4 ROCOP Mechanistic discussion}

By considering all of the kinetic data we have obtained, together with that published elsewhere, some details around the general ROCOP mechanism can be summarised. ${ }^{17,}{ }^{18}$ Initial reaction rates are not affected by the amount of anhydride consumption, suggesting a zero order dependence on anhydride concentration. The initiation is deduced to be first order with respect to both catalyst concentration and epoxide concentration. Therefore the rate expression for this process can be expressed as: $r a t e=k$ [catalyst][epoxide]. For the initiation step, MALDI-ToF mass spectrometry is useful for identifying possible end-groups for polymer chains, which are effectively also the initiating groups for starting polymerisations, i.e. the anion which first attacks and ring-opens an epoxide coordinated to a metal.

The propagation step, however, is more complicated to investigate compared to the initiation, and with respect to studying plausible mechanisms. The simplest, or most ideal, possibility is for the opened epoxide to attack a coordinated anhydride molecule, with the epoxide tether now disassociating from the metal. This is followed by a new epoxide molecule coordinating, which is in turn attacked by the now ring-opened anhydride, leaving space for a new anhydride molecule and the process repeats. All of this could occur around a single metal center, or a "chain-shuttling" mechanism could exist based upon two proximal metal centers - the latter is depicted in Figure S28. The propagation step can be further complicated by the presence of competing processes such as transesterification and ether insertions. 


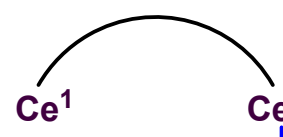<smiles>C[Ge]OC1CCCCC1OC(=O)P</smiles>
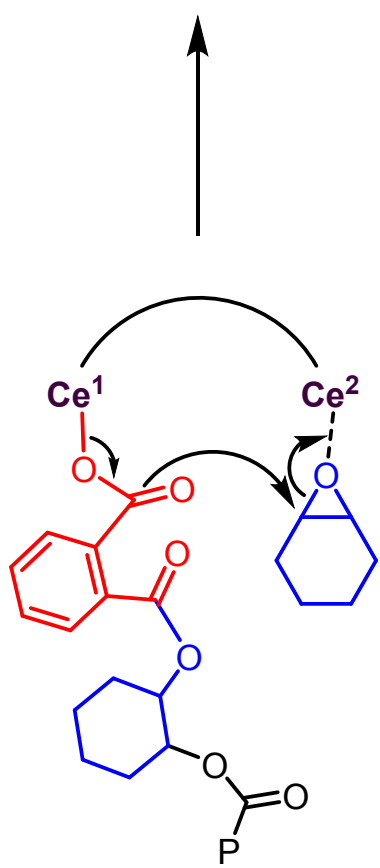
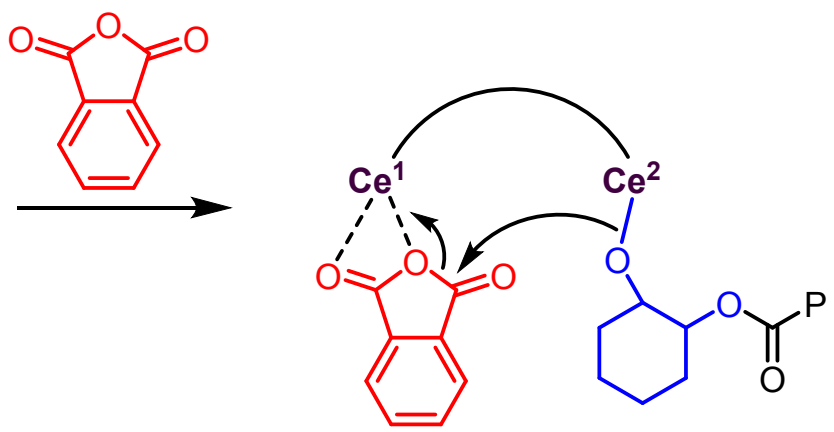
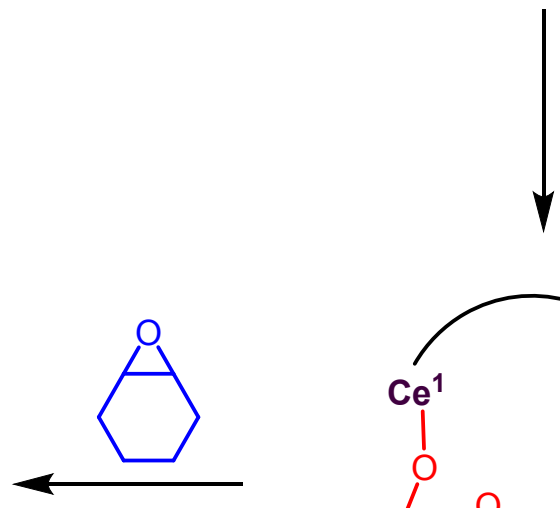

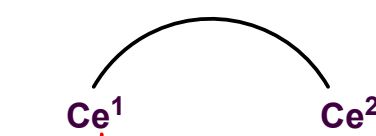

Figure S34: Plausible mechanism for propagation step that involves metal-metal cooperativity. 


\section{S3.5 SEC Traces}

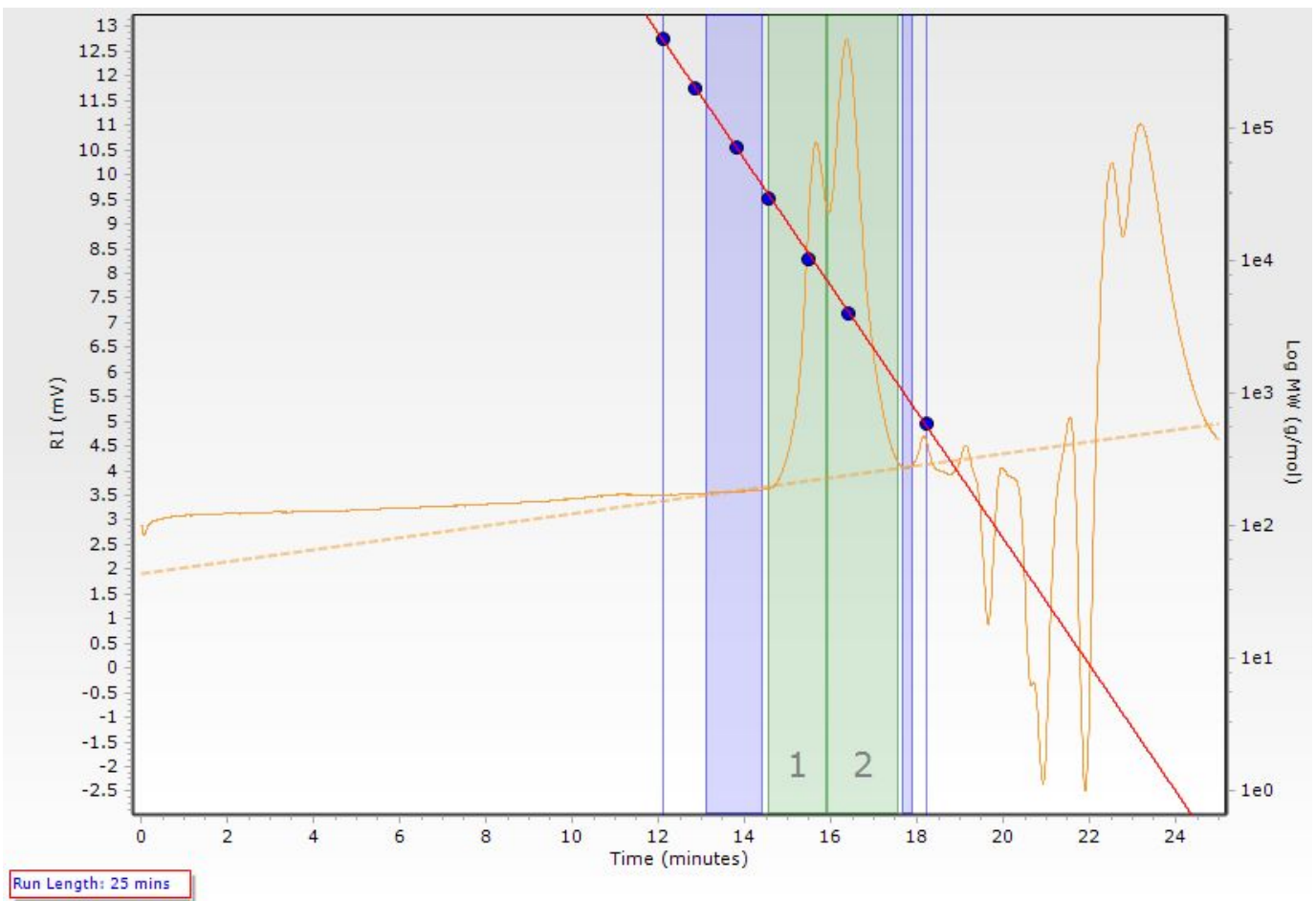

Figure S35: SEC trace for PA/CHO co-polyester formed by 1- $\boldsymbol{m}$ - $^{\mathrm{t}} \mathrm{Bu}$ (Table S3 Entry 3).

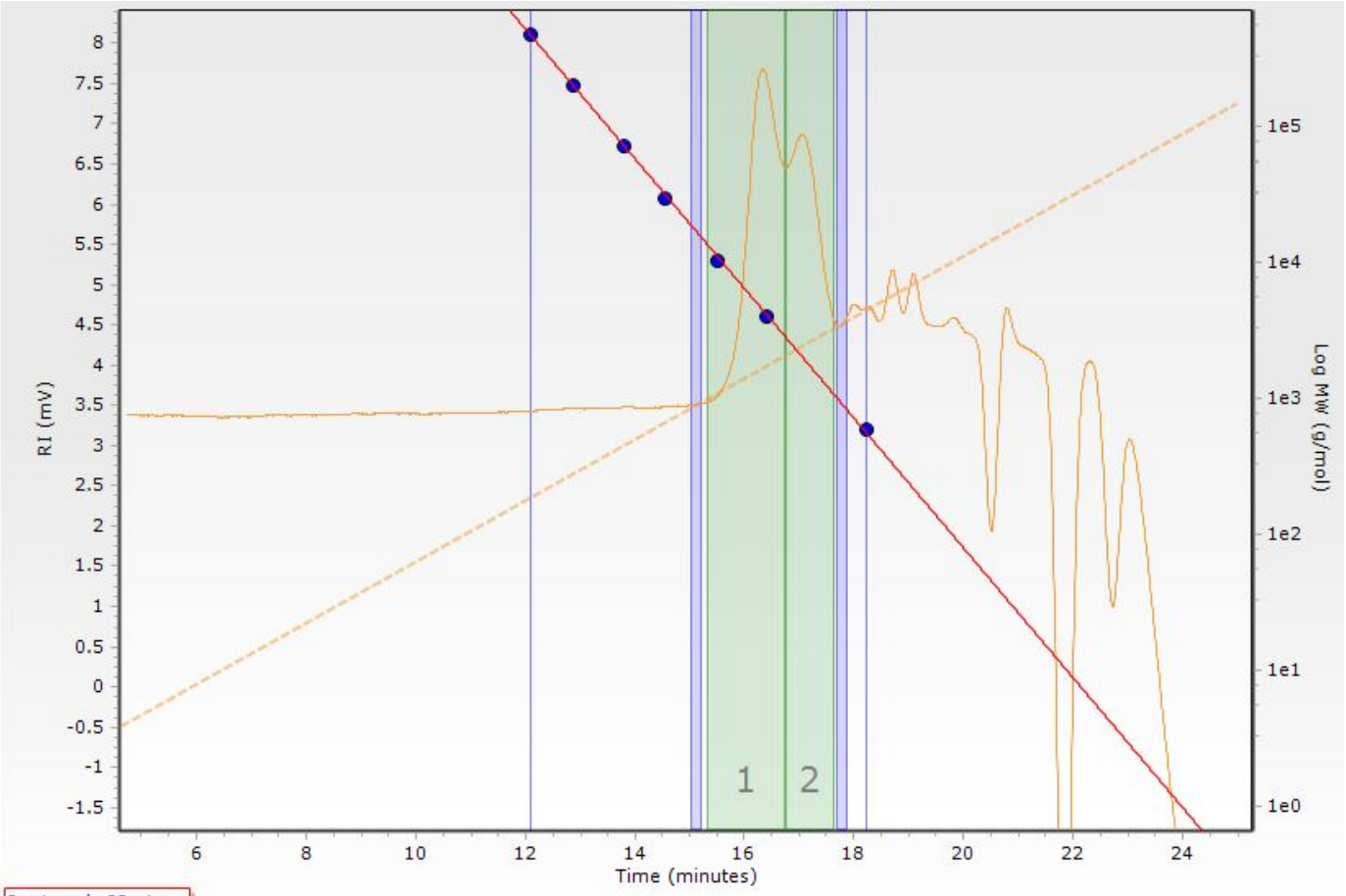

Run Length: 25 mins

Figure S36: SEC trace for PA/CHO co-polyester formed by 1-p-'Bu (Table S3 Entry 4). 


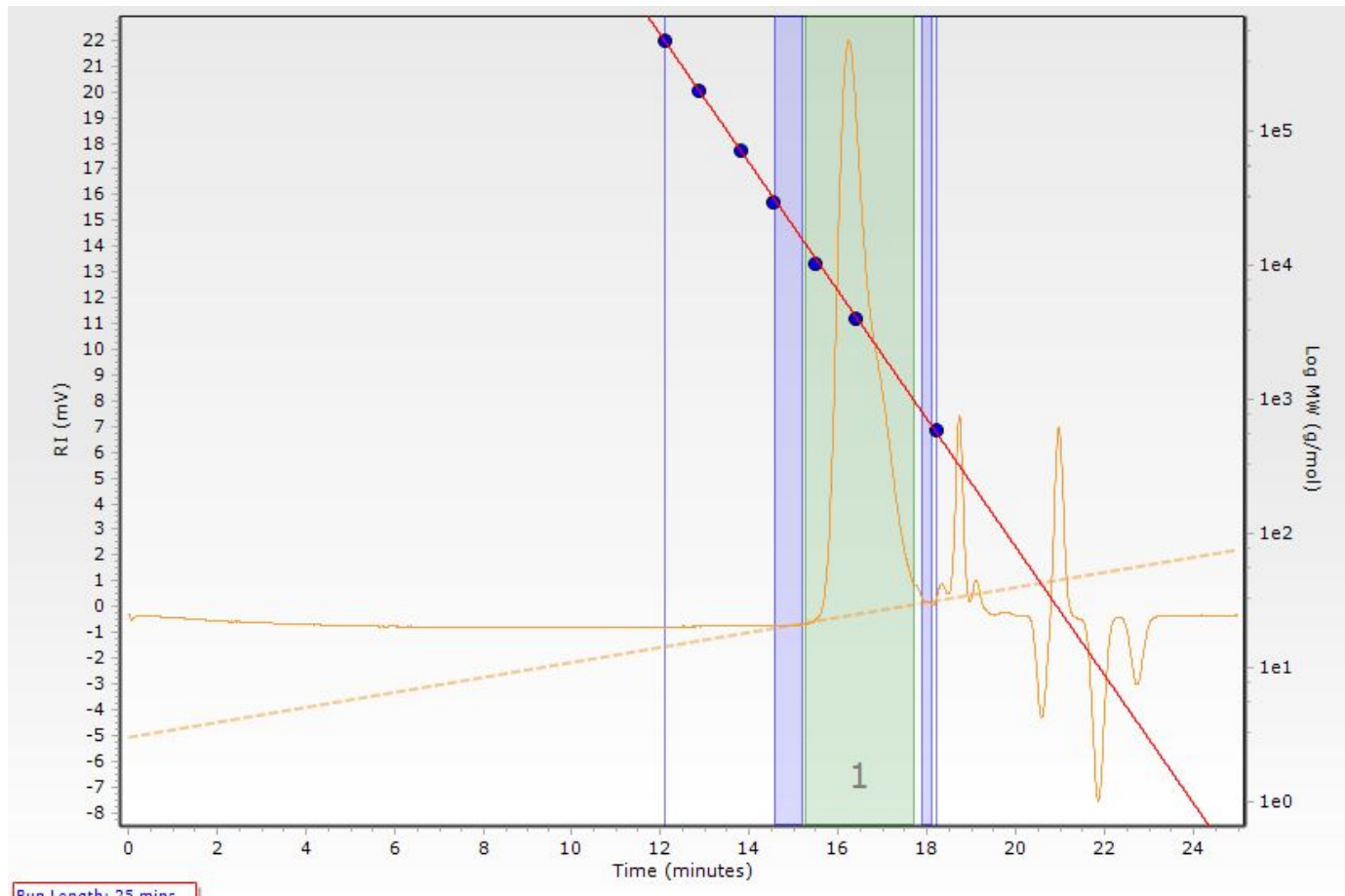

Run Length: 25 mins

Figure S37: SEC trace for PA/CHO co-polyester formed by 1-p-'Bu (Table S3 Entry 5).

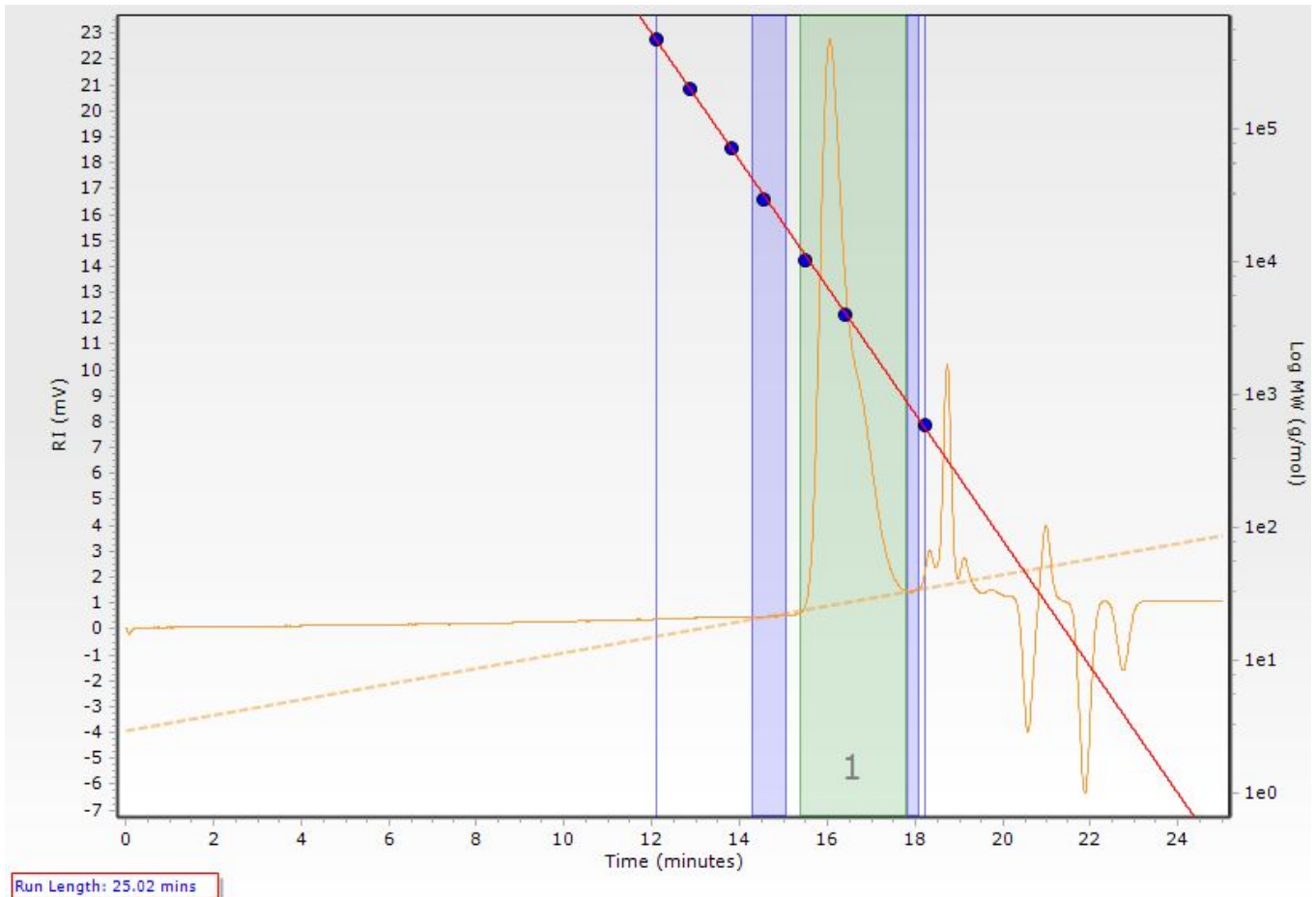

Figure S38: SEC trace for PA/CHO co-polyester formed by 1-p- ${ }^{\mathrm{t}} \mathrm{Bu}$ (Table S3 Entry 6). 


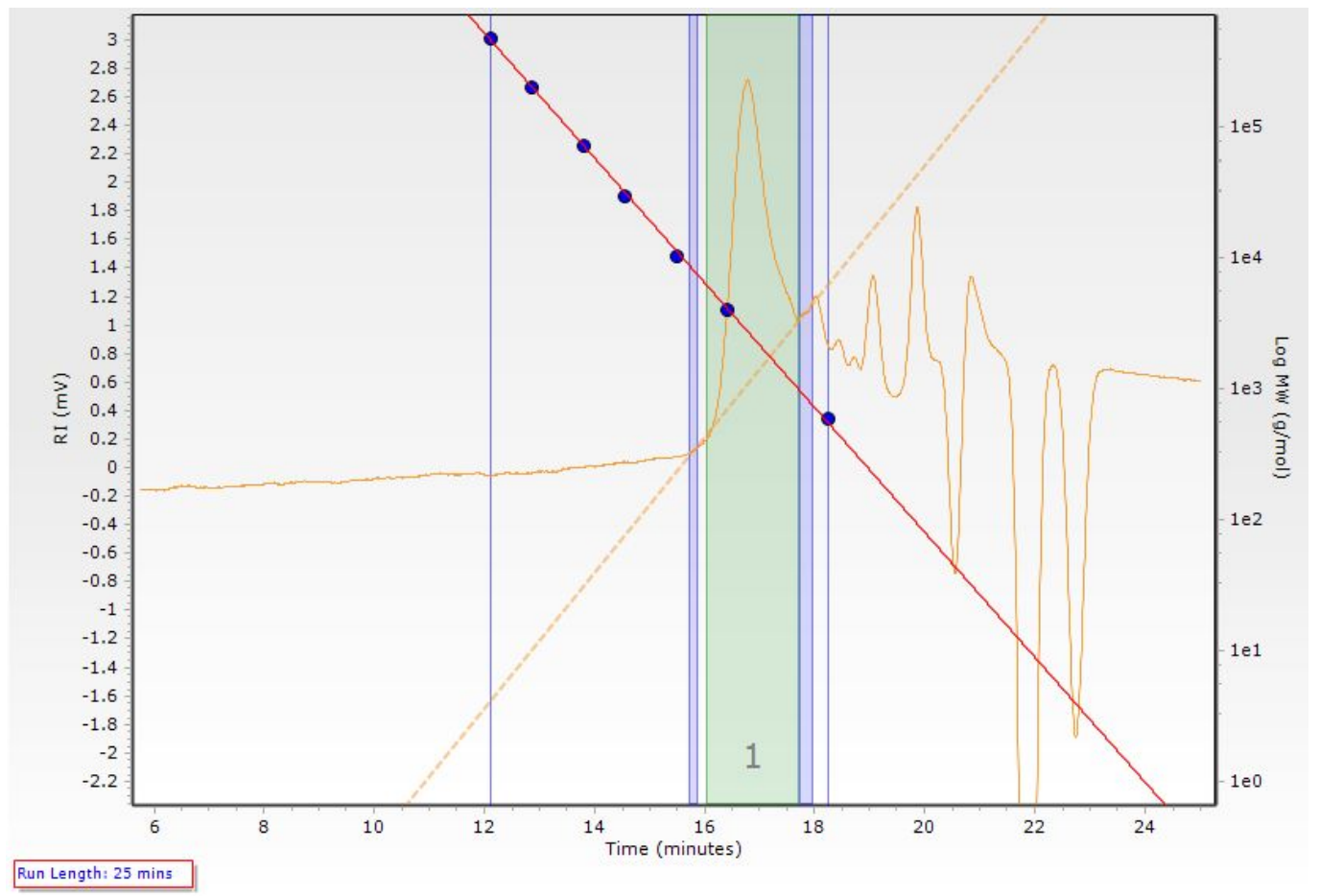

Figure S39: SEC trace for PA/CHO co-polyester formed by 1-m-Ad (Table S3 Entry 9).

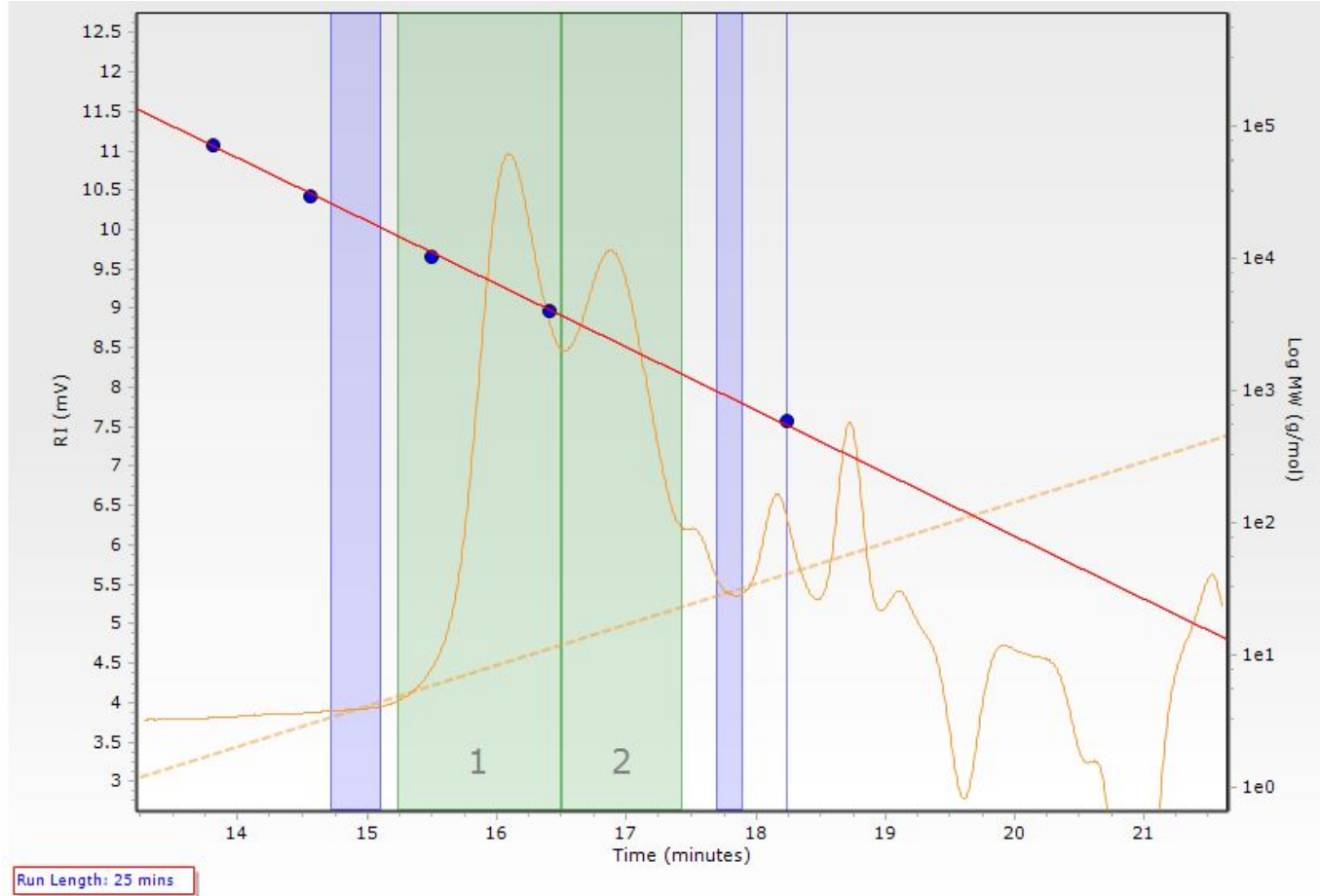

Figure S40: SEC trace for PA/CHO co-polyester formed by 1-p-Ad (Table S3 Entry 11). 


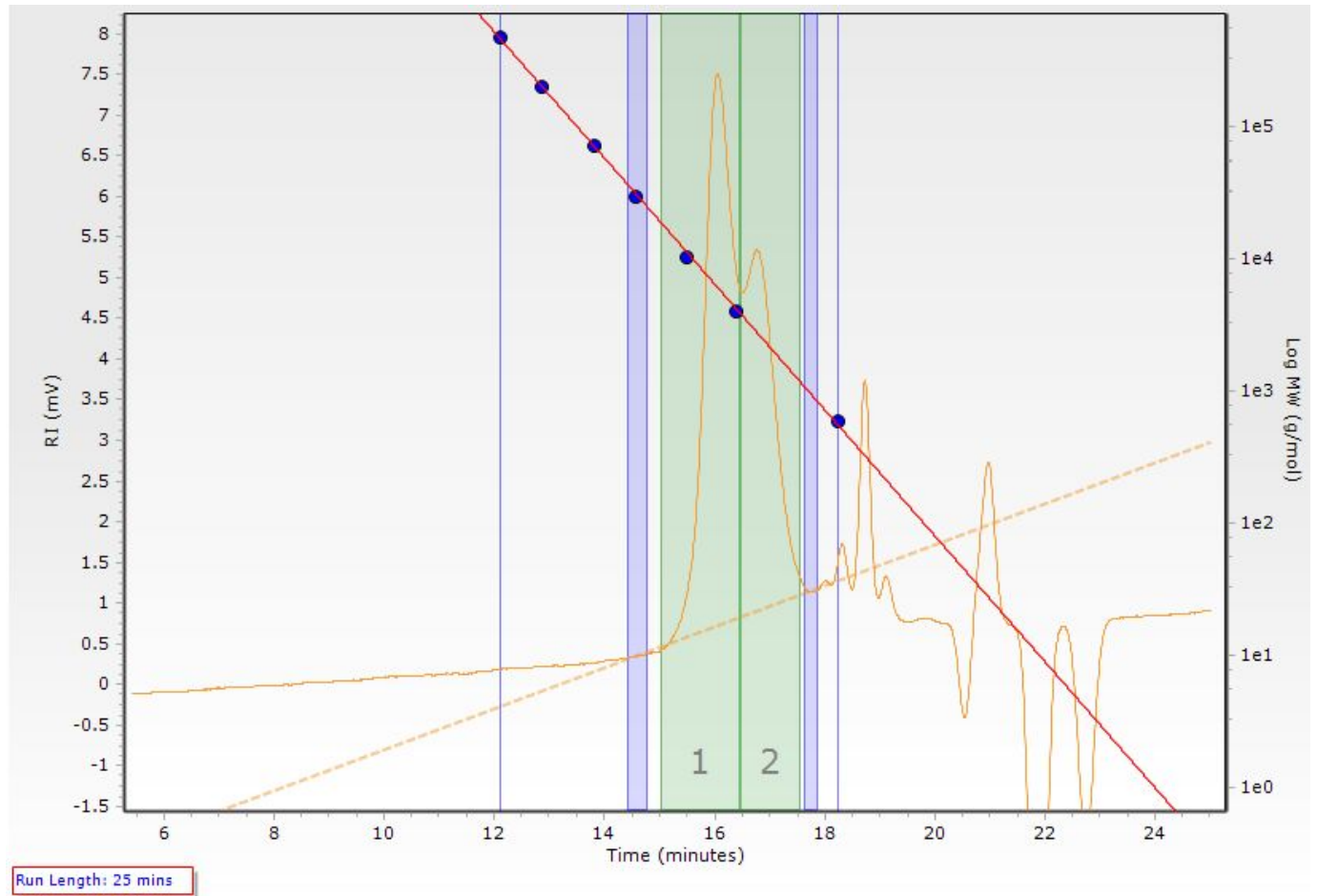

Figure S41: SEC trace for PA/CHO co-polyester formed by 1-p-Ad (Table S3 Entry 12).

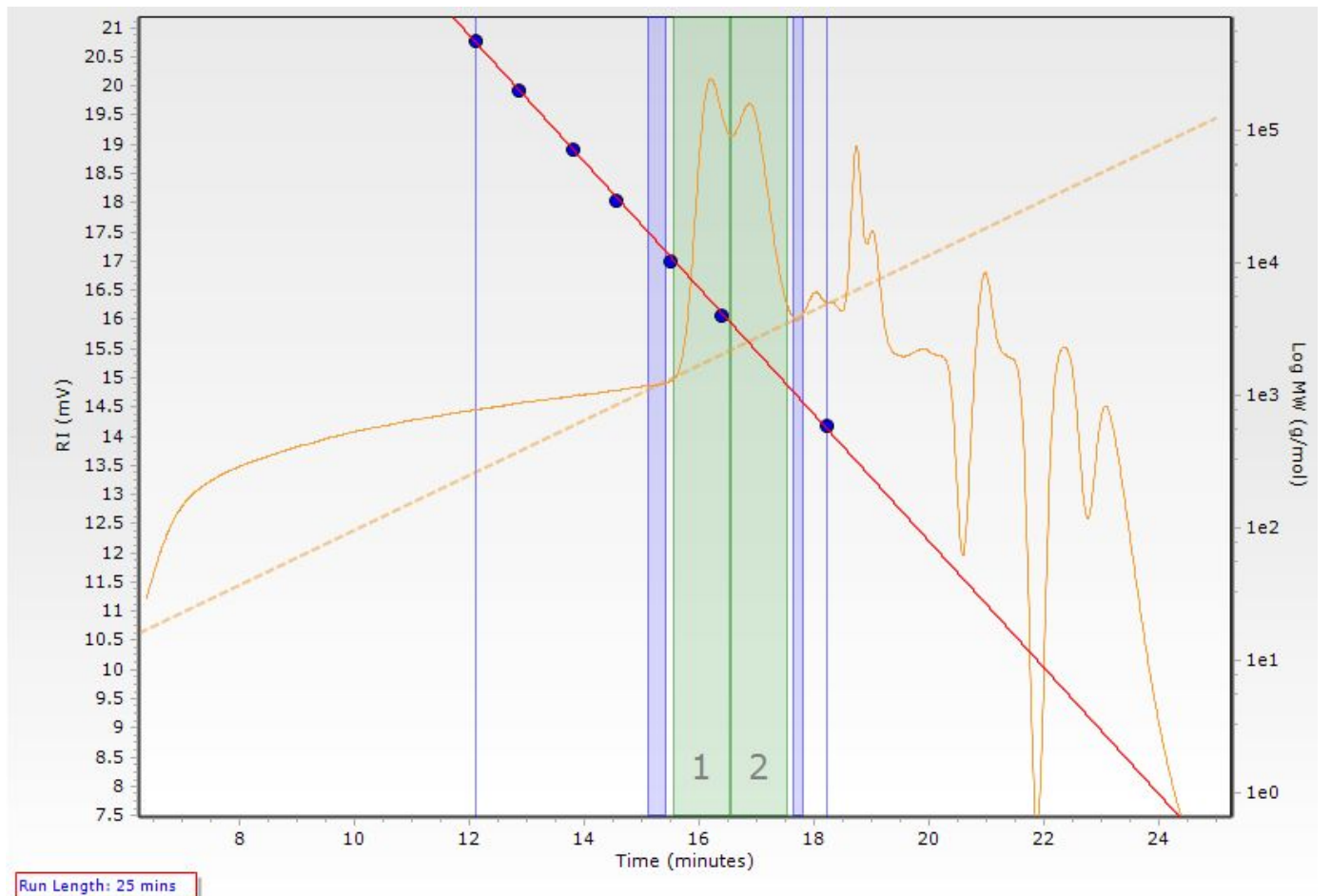

Figure S42: SEC trace for PA/CHO co-polyester formed by 2-p-'Bu (Table S3 Entry 15). 


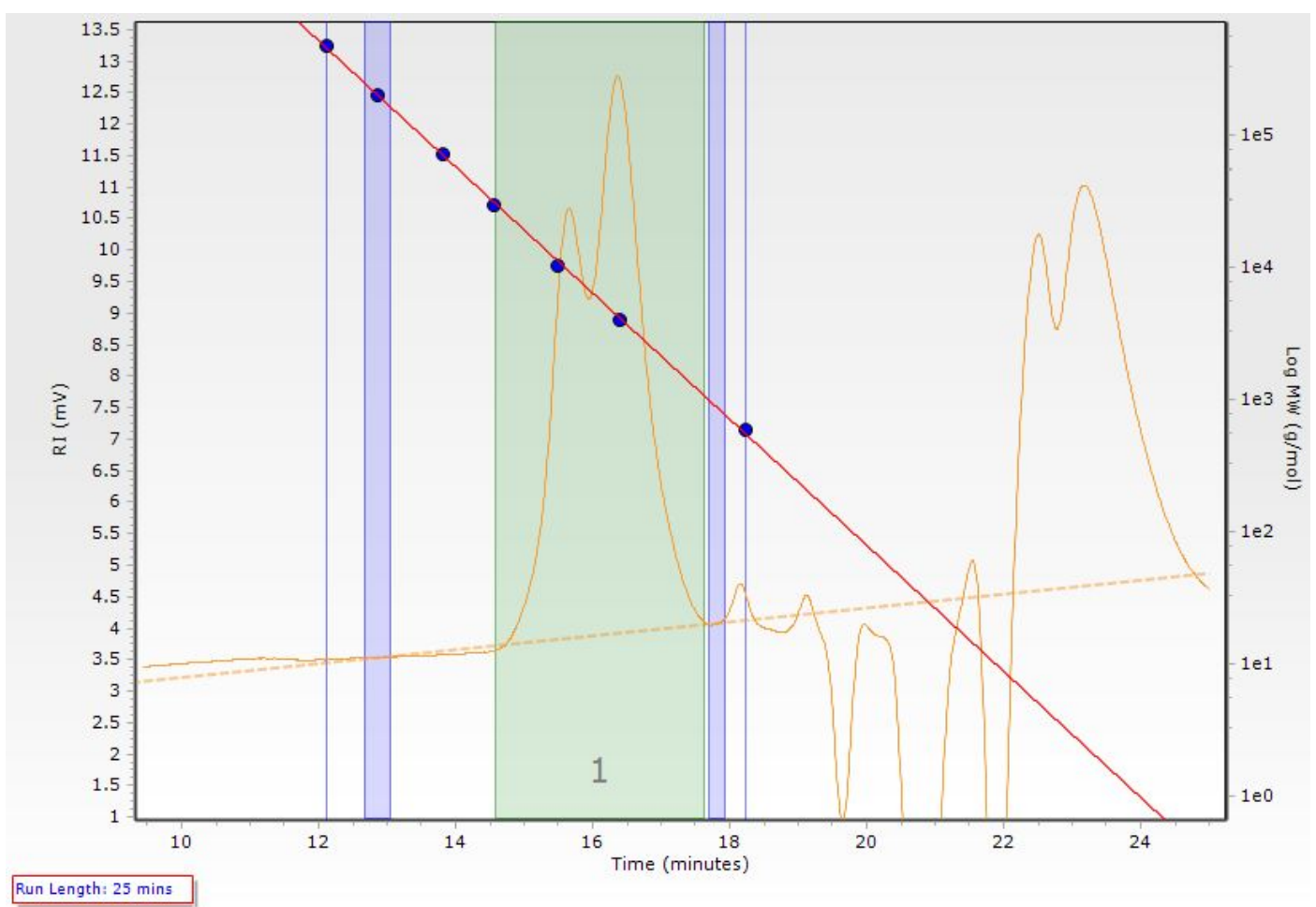

Figure S43: SEC trace for PA/CHO co-polyester formed by 3 (Table S3 Entry 18).

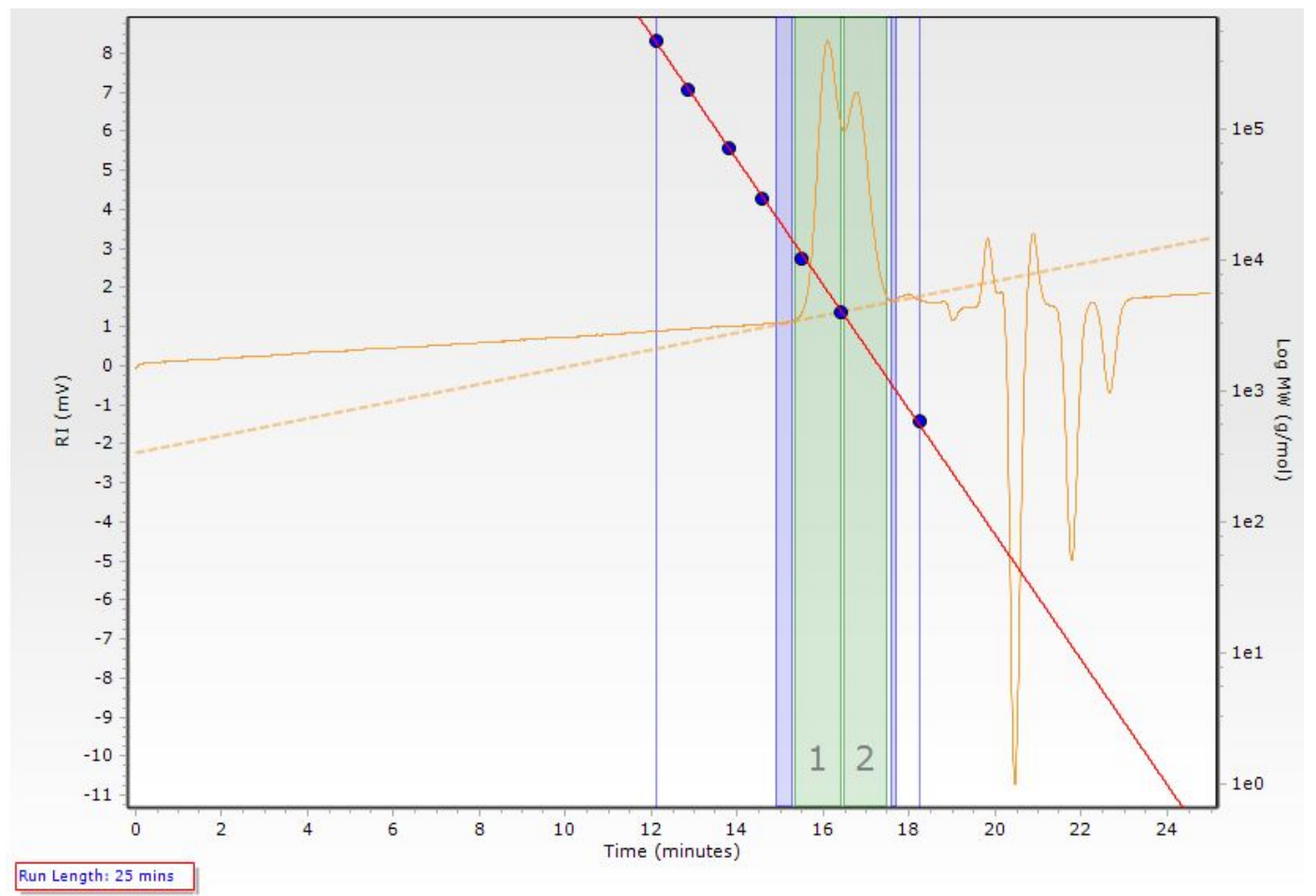

Figure S44: SEC trace for PA/CHO co-polyester formed by 4 (Table S3 Entry 20). 


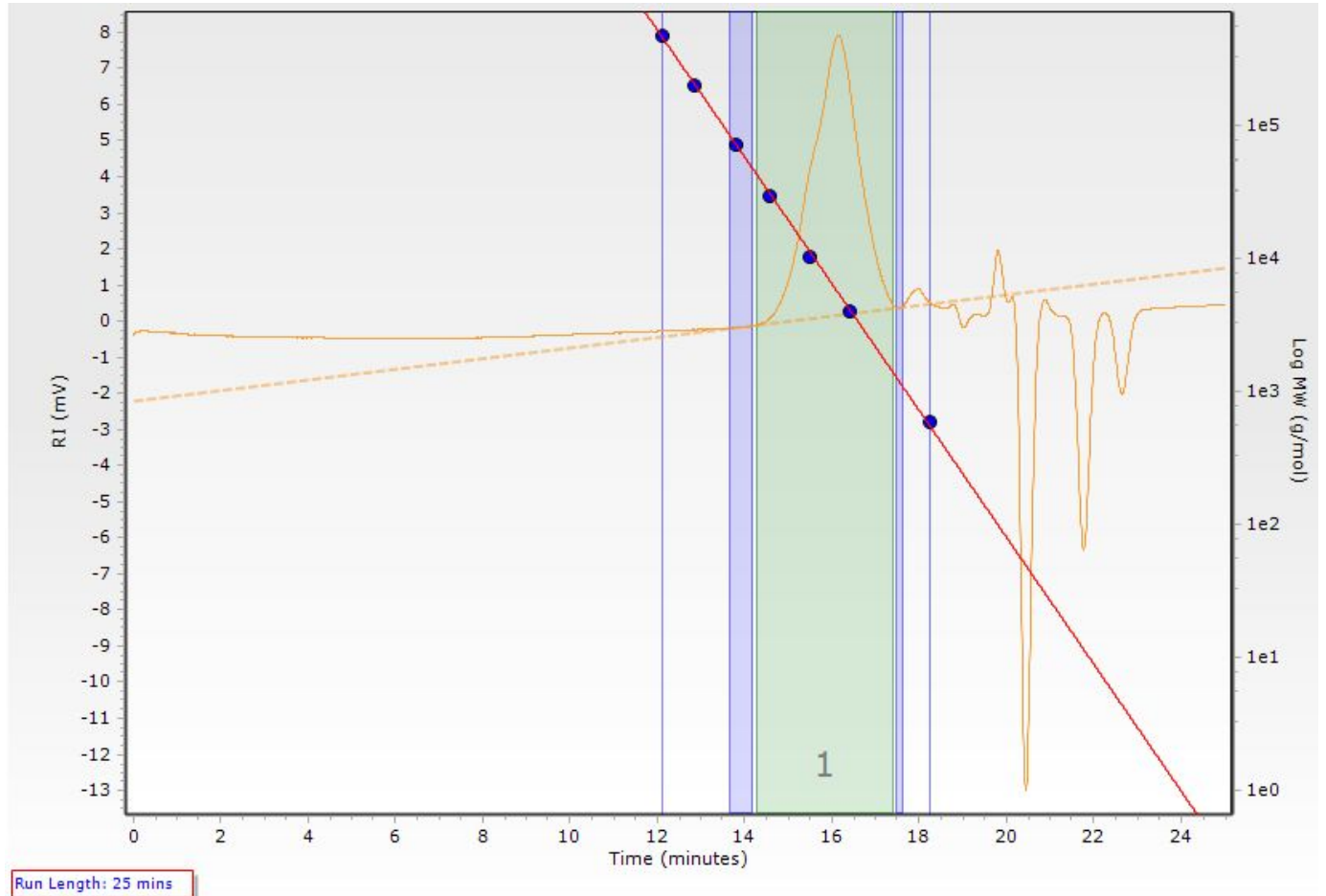

Figure S45: SEC trace for PA/CHO co-polyester formed by $\mathbf{5}$ (Table S3 Entry 22).

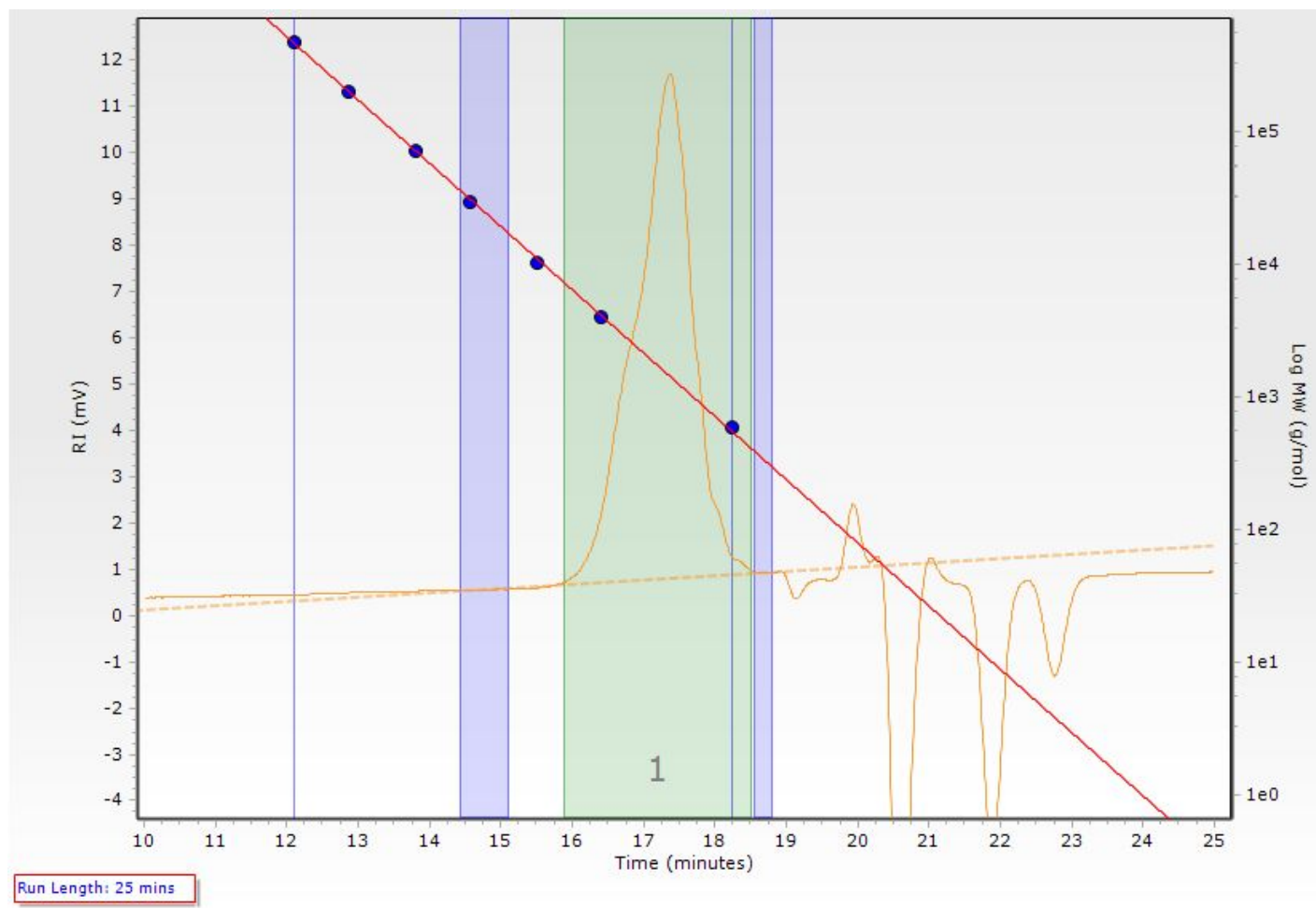

Figure S46: SEC trace for PA/CHO co-polyester formed by $\left[\mathrm{NEt}_{4}\right]_{2} \mathrm{CeCl}_{6}$ (Table S3 Entry 23). 


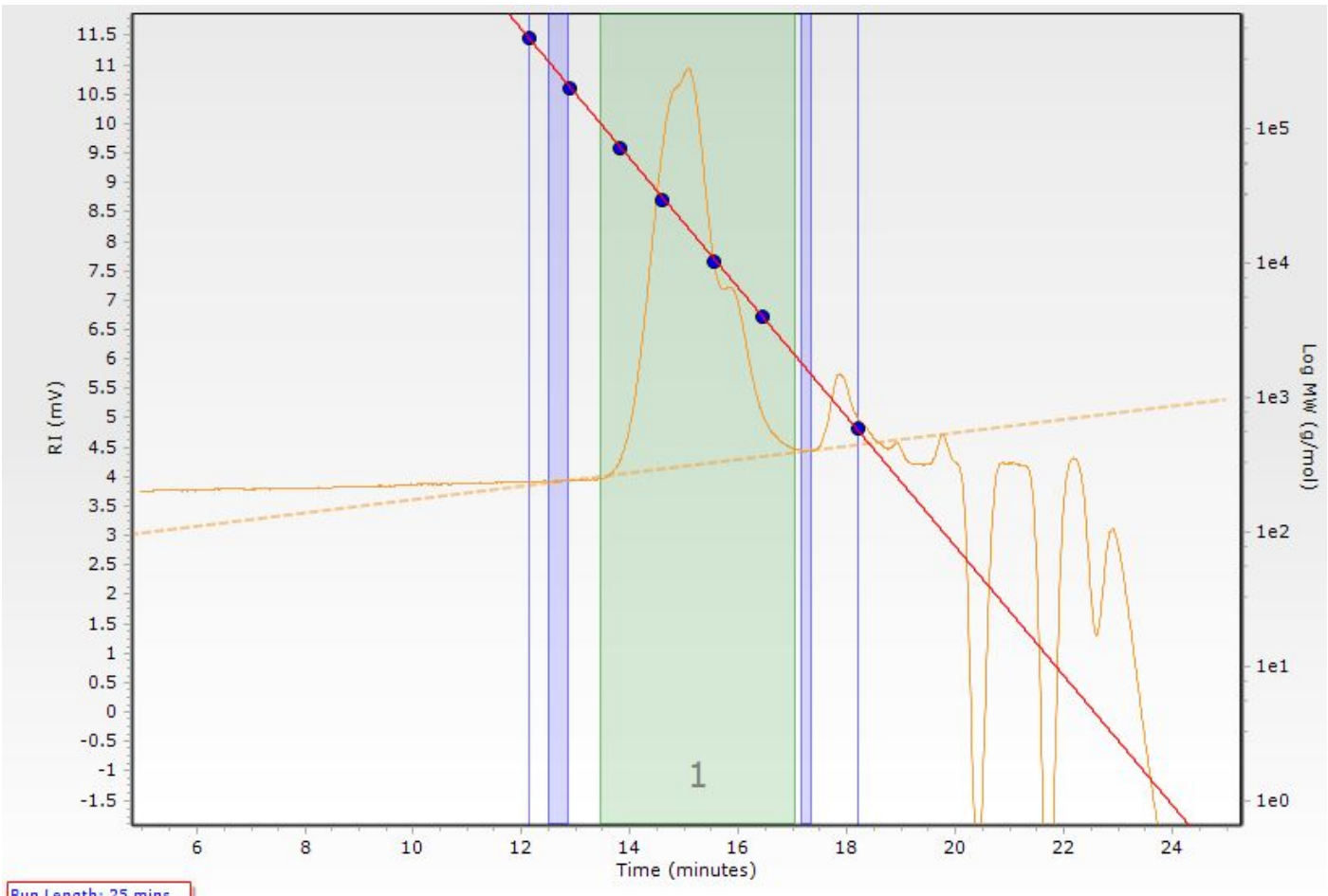

Run Length: 25 mins

Figure S47: SEC trace for PA/PO co-polyester formed by 1-m-Ad (Table S5 Entry 2).

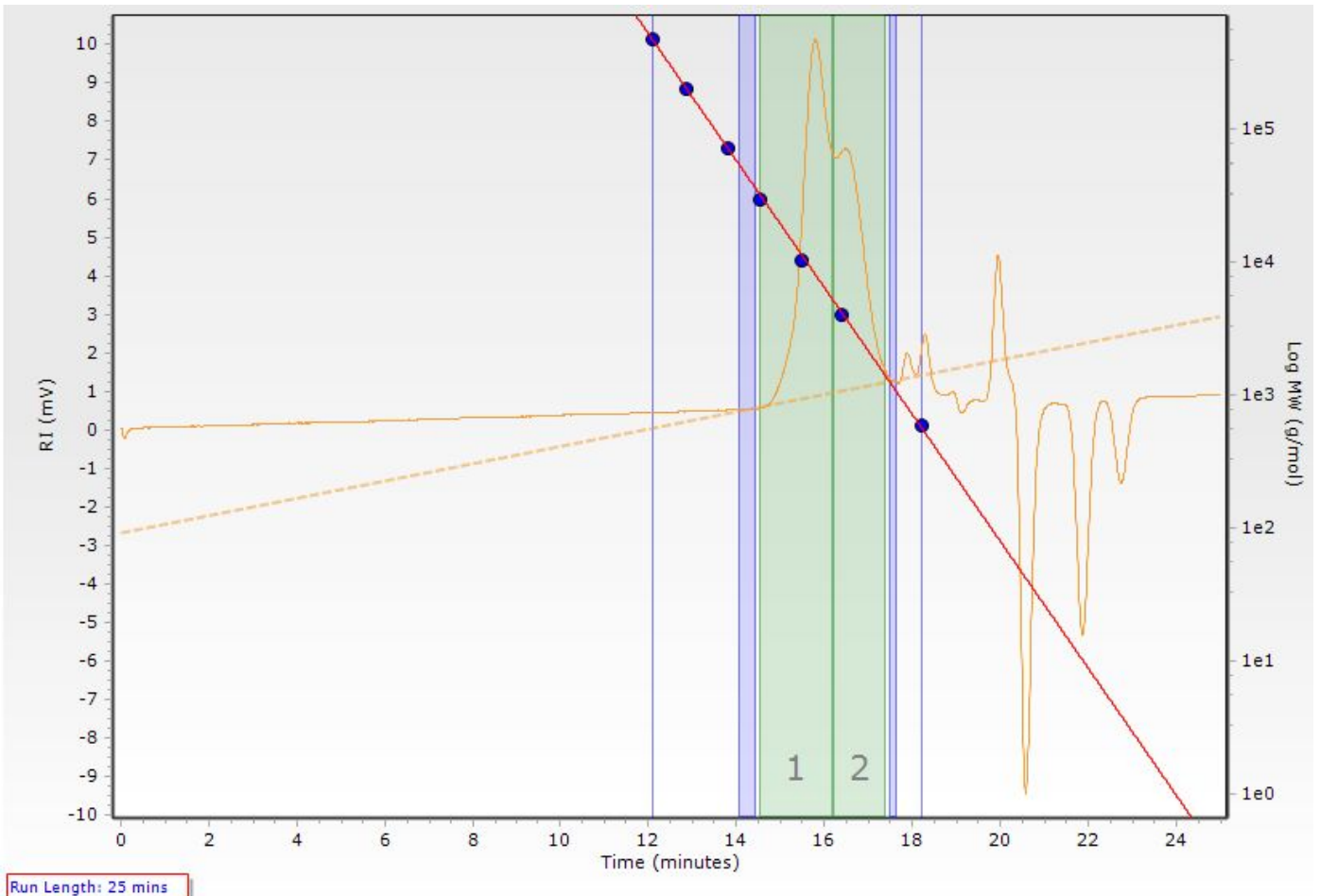

Figure S48: SEC trace for PA/LO co-polyester formed by 1-m-Ad (Table S5 Entry 3). 


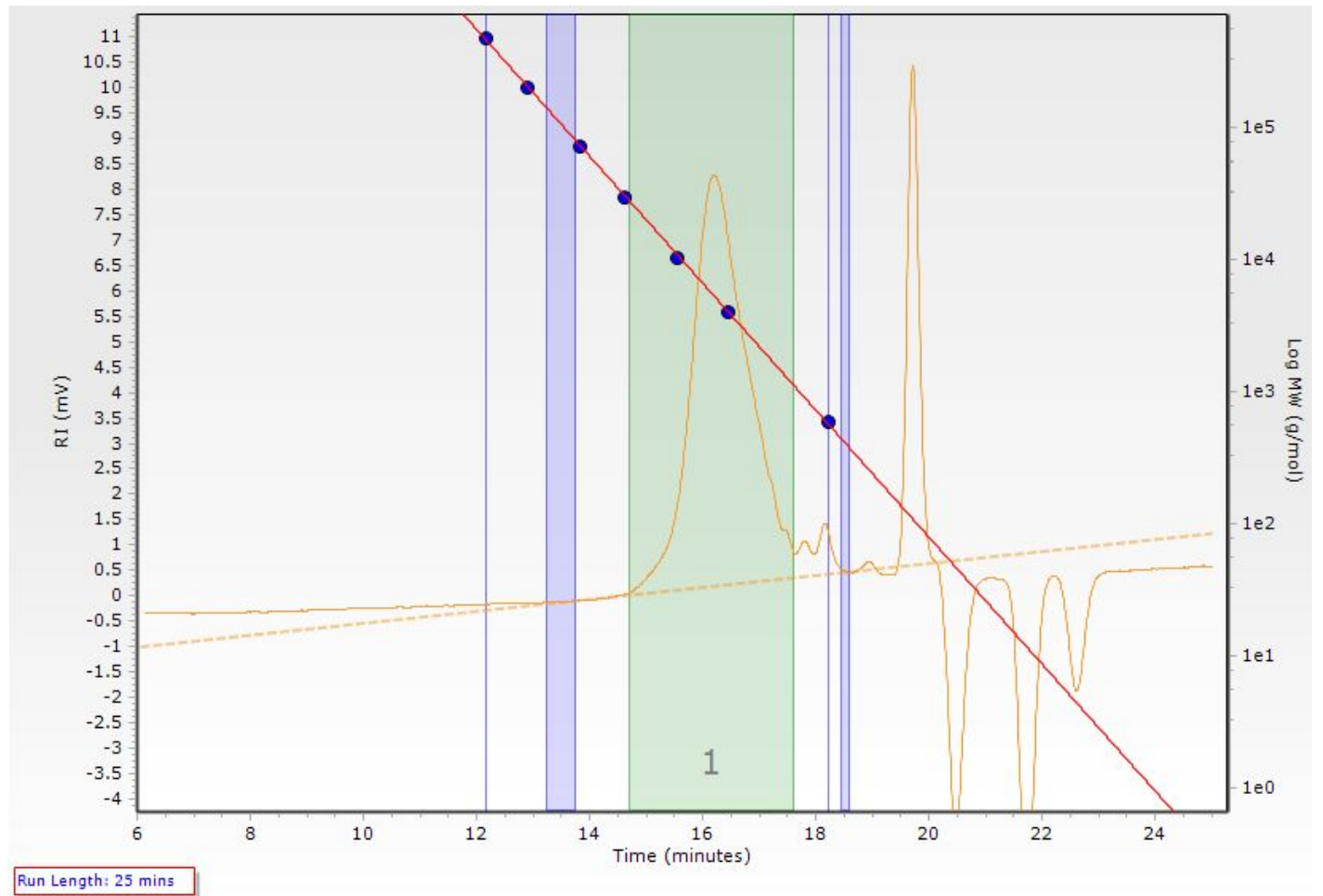

Figure S49: SEC trace for SA/CHO co-polyester formed by 1-m-Ad (Table S5 Entry 5).

\section{S3.6 MALDI Data}

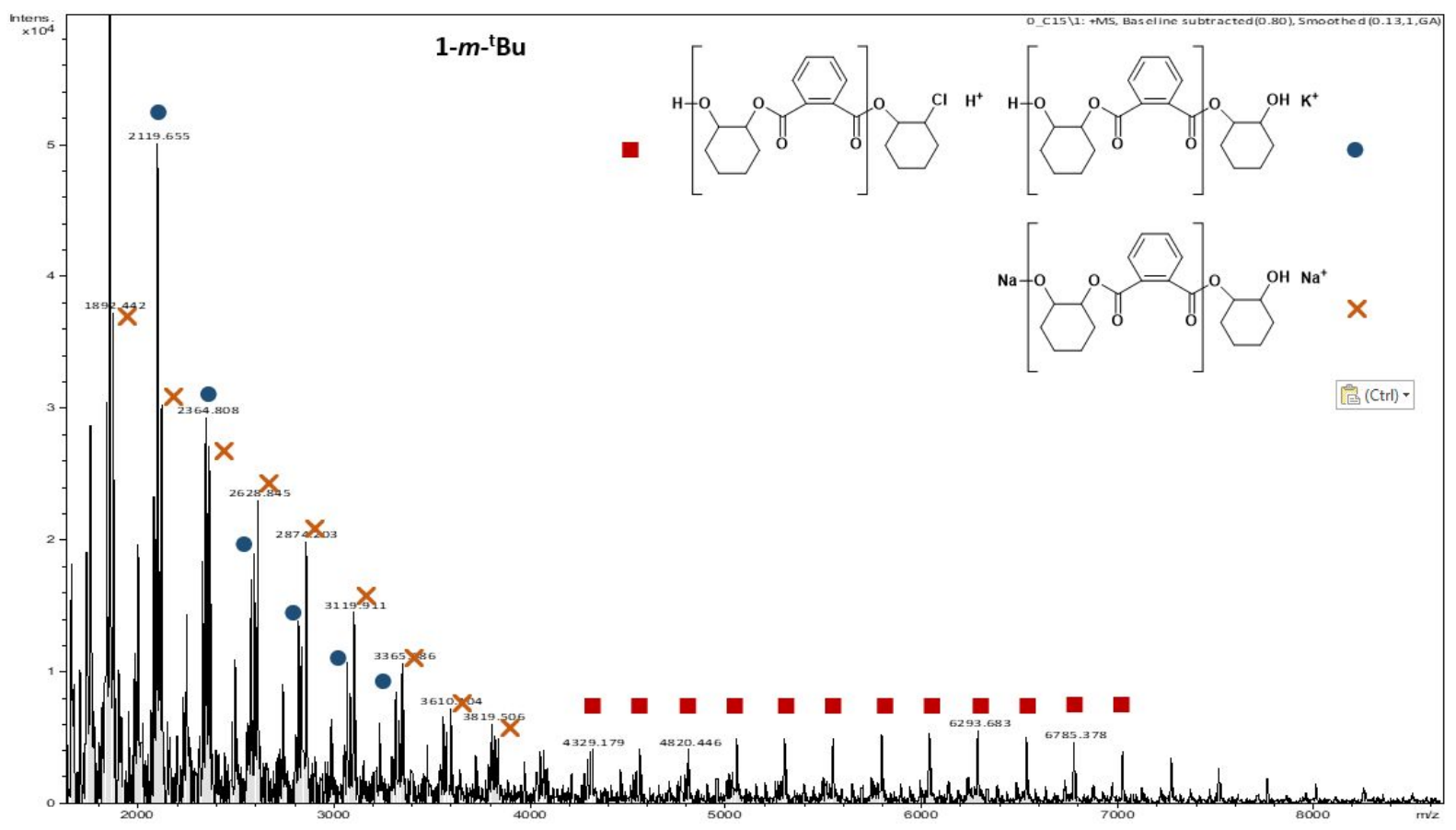

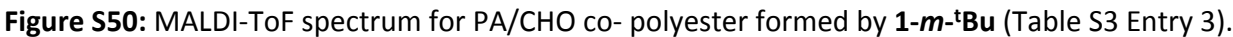




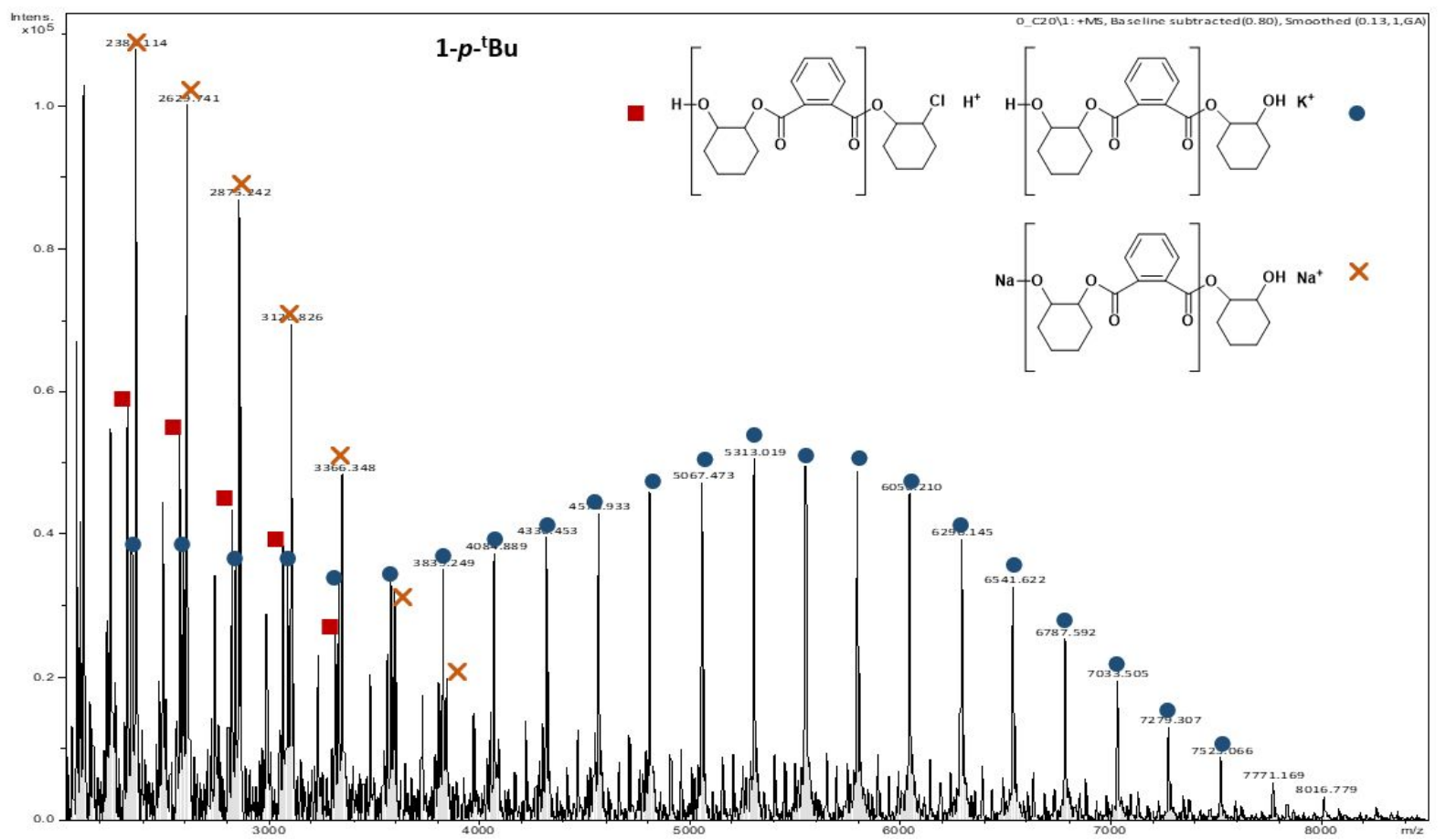

Figure S51: MALDI-ToF spectrum for PA/CHO co-polyester formed by 1-p-'Bu (Table S3 Entry 5).

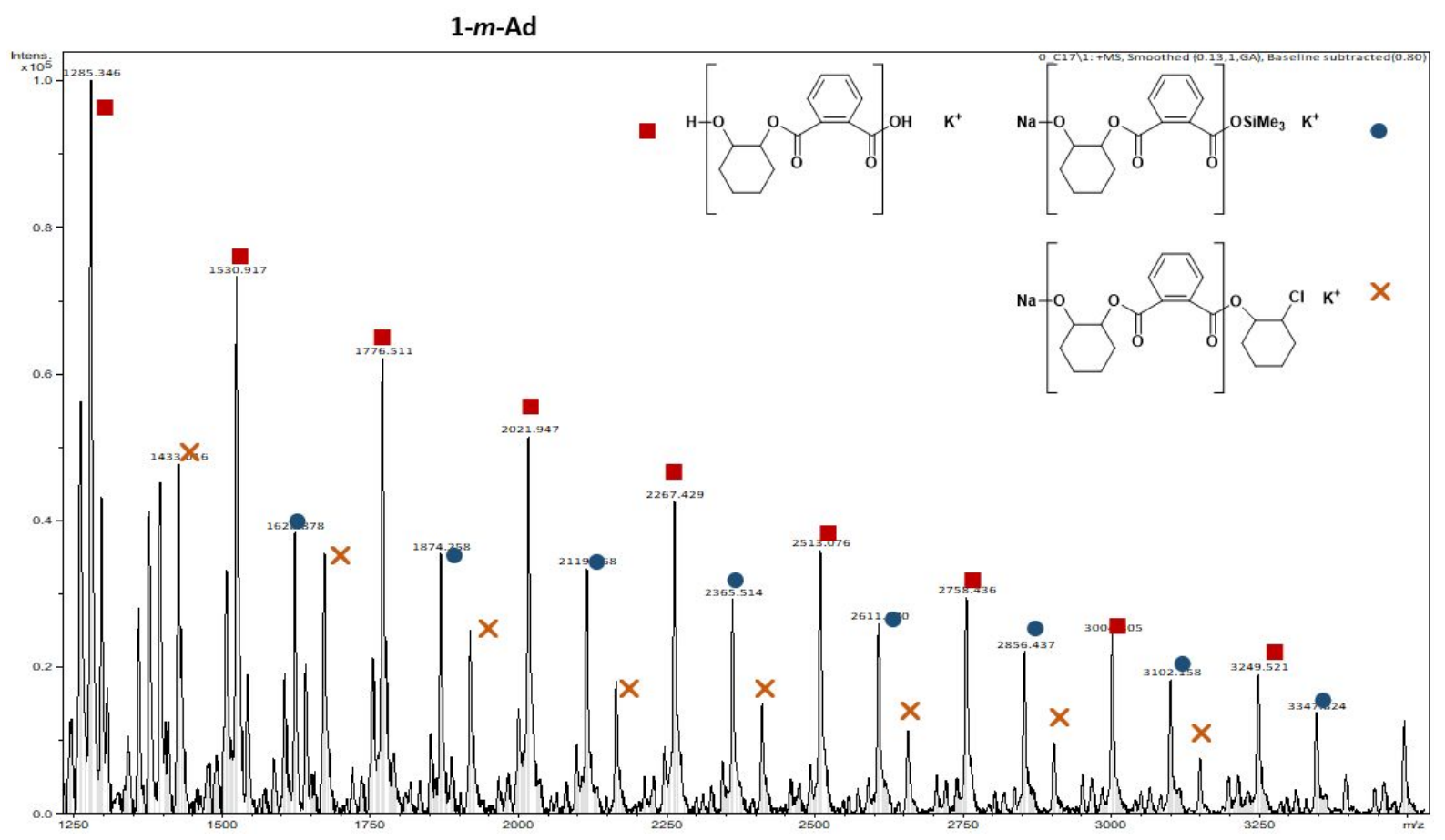

Figure S52: MALDI-ToF spectrum for the PA/CHO co-polyester formed by 1-m-Ad (Table S3 Entry 9). 


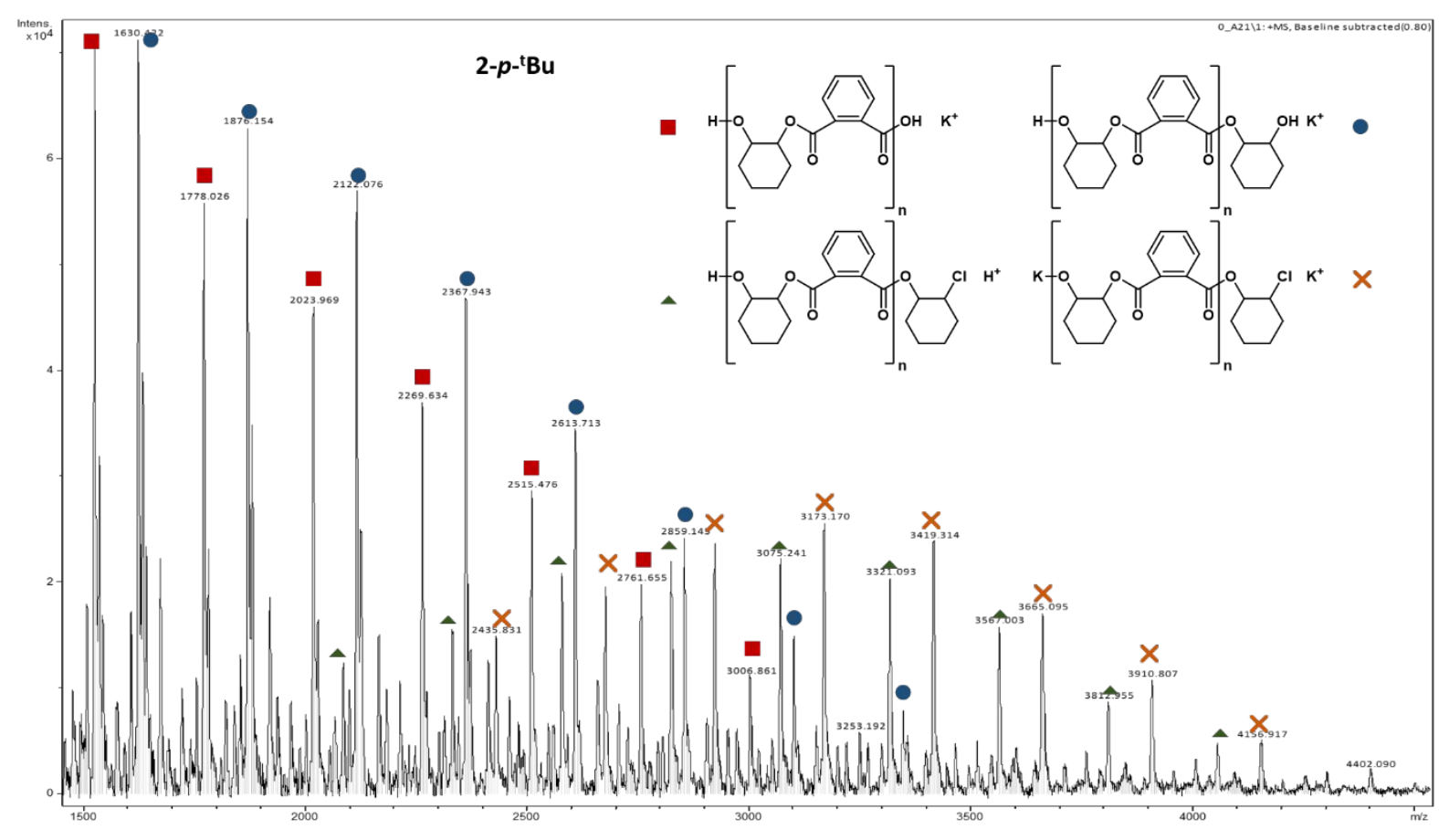

Figure S53: MALDI-ToF spectrum for the PA/CHO co-polyester formed by 2-p-tBu (Table S3 Entry 13).

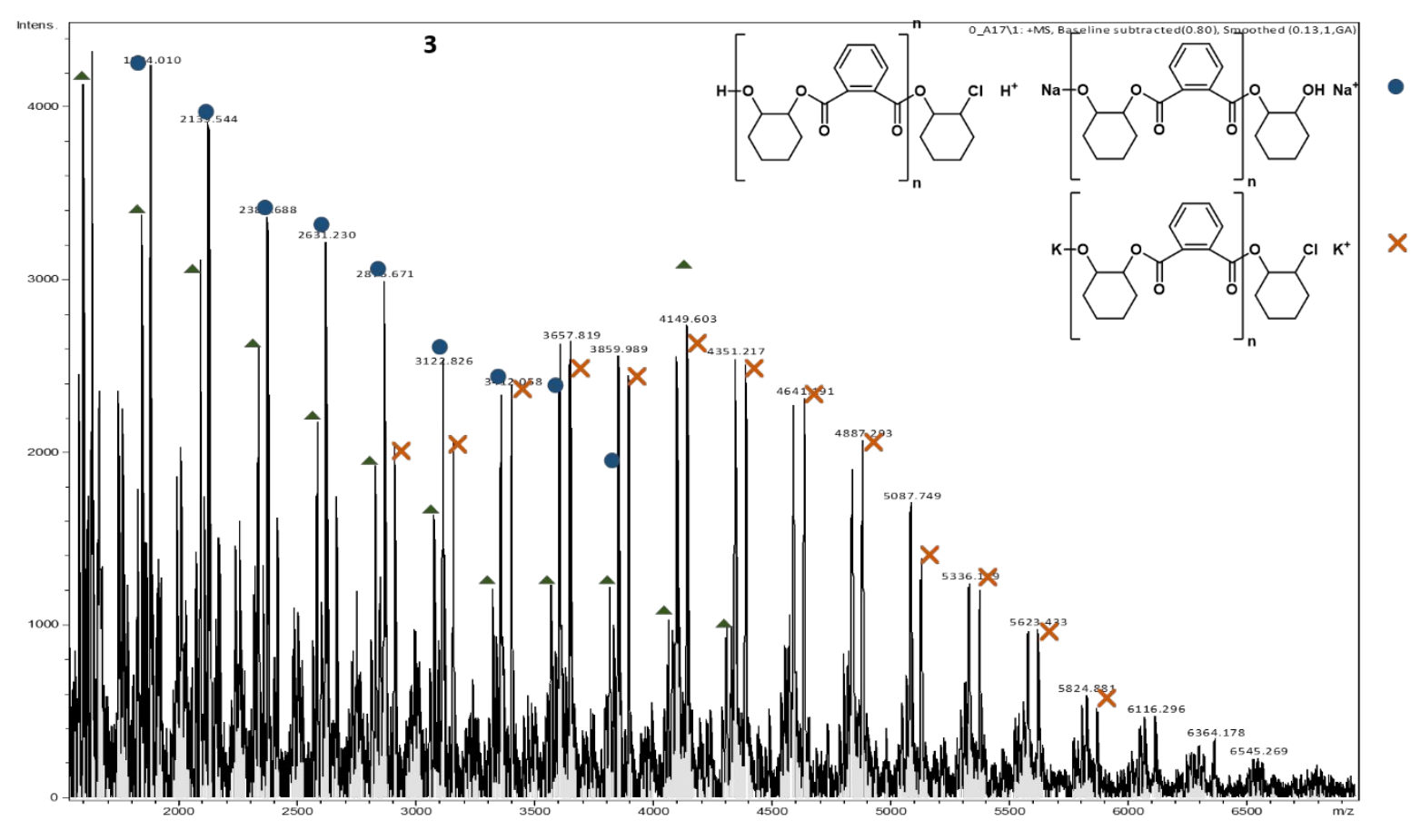

Figure S54: MALDI-ToF spectrum for PA/CHO co-polyester formed by 3 (Table S3 Entry 17). 


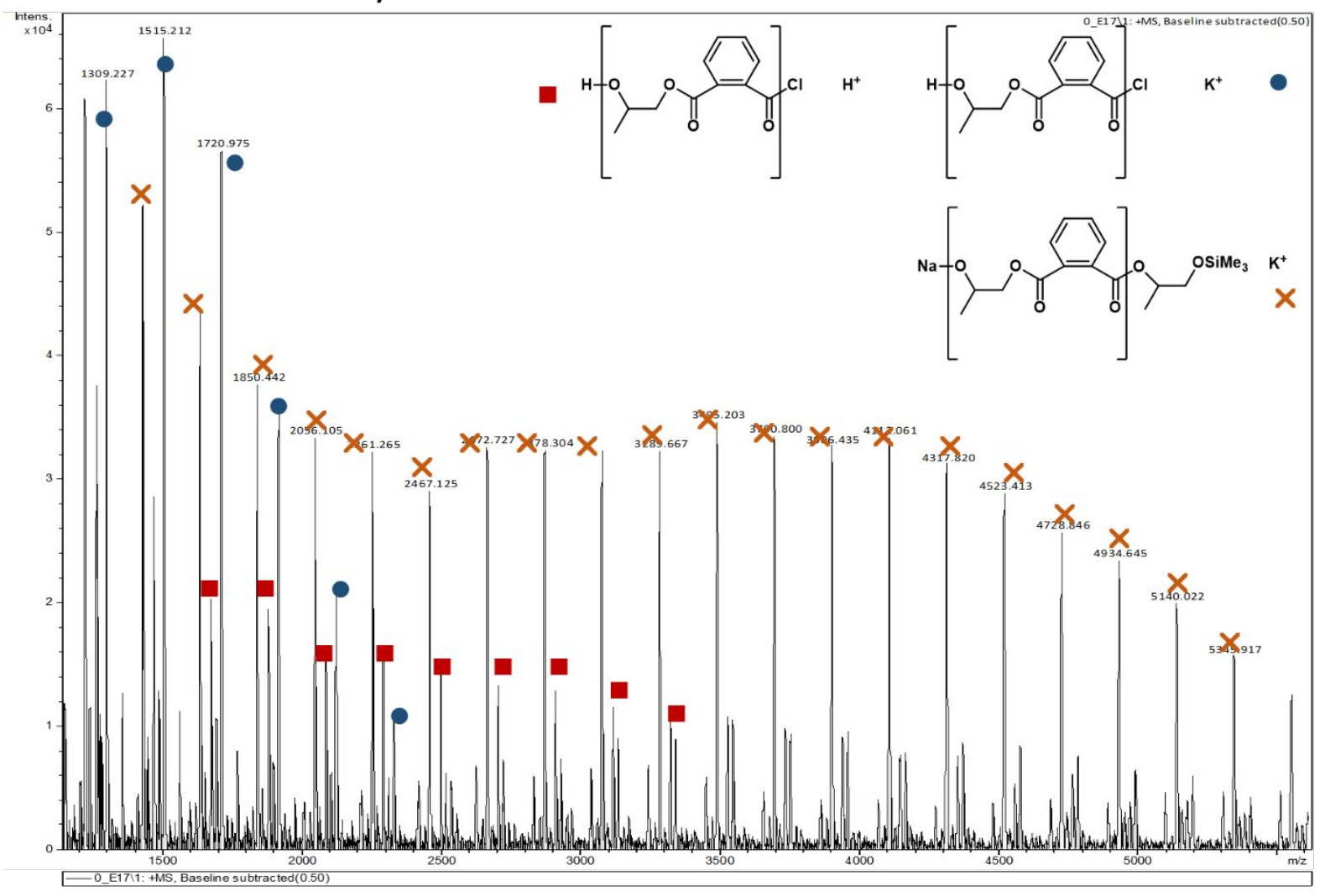

Figure S55: MALDI-ToF spectrum for PA/PO co-polyester formed by 1-m-Ad (Table S4 Entry 1).

\section{SA/PO}

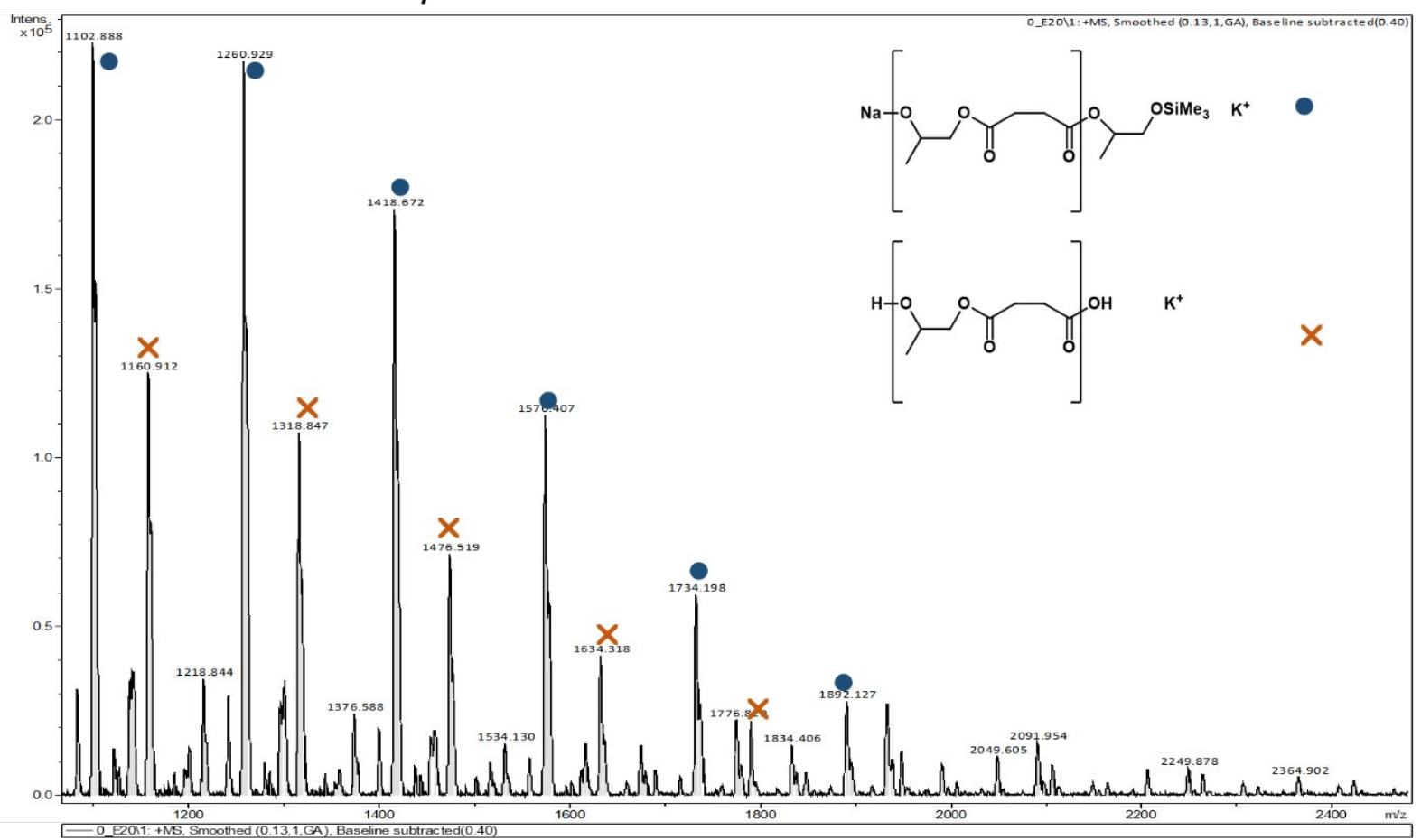

Figure S56: MALDI-ToF spectrum for SA/PO co-polyester formed by 1-m-Ad (Table S4 Entry 4). 


\section{S4. Crystallography}

Single crystal X-ray diffraction data were collected using an Excalibur Eos diffractometer, fitted with a CCD area detector and using MoKa radiation $\left(\lambda=0.71073 \AA\right.$ ) at $190 \mathrm{~K}$. The molecular structure of $\mathbf{1}^{*}$-p-Ad was solved using SHELXT ${ }^{19}$ and least-square refined using SHELXL ${ }^{20}$ in Olex2. ${ }^{21}$ Hydrogen atoms were treated by constrained refinement.

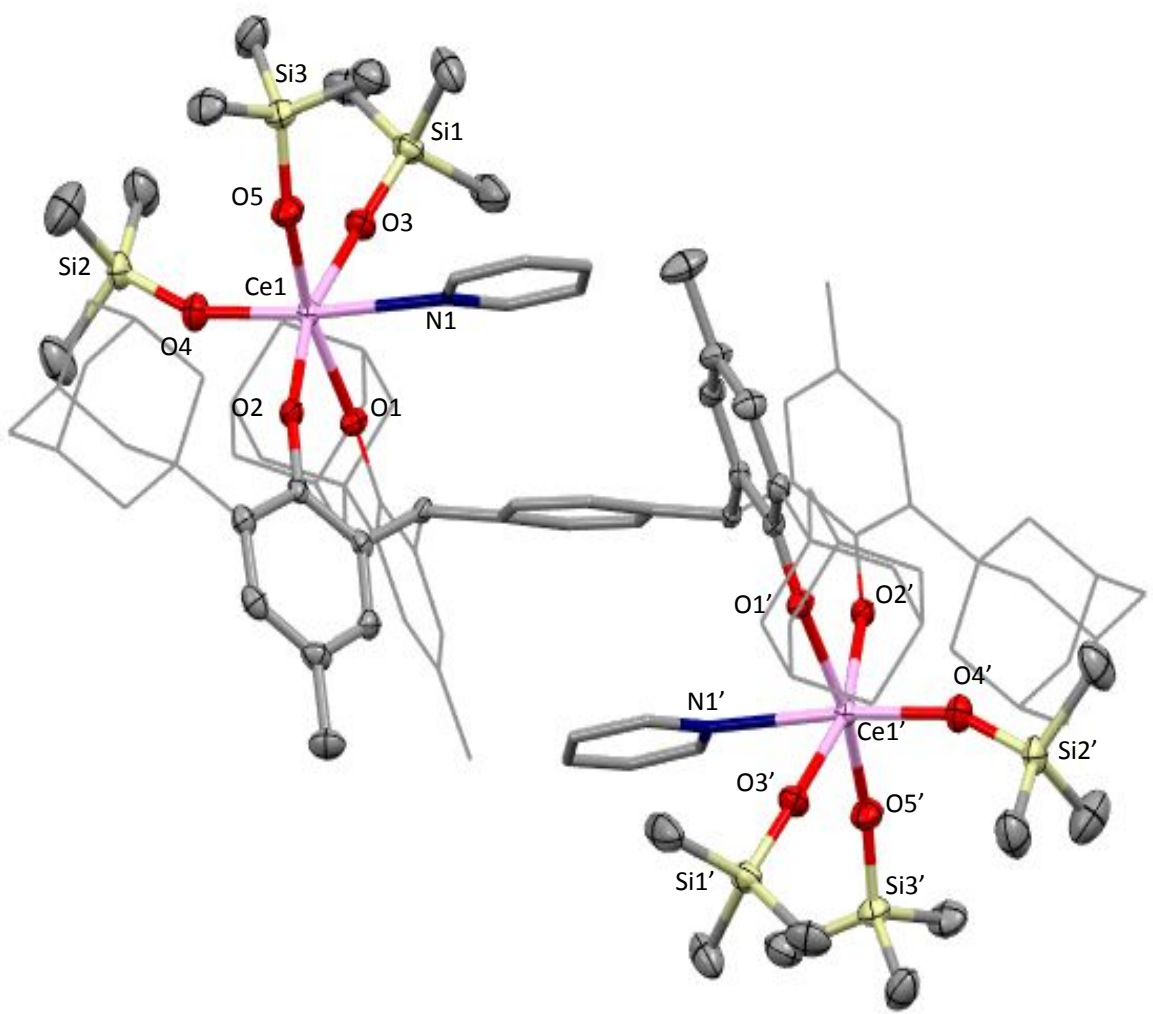

Figure S57: Solid-state structure of the dianion [1*-p-Ad] $]^{2-}$ with selected $\mathrm{C}$ and non-C/H atoms shown at $50 \%$ ellipsoid probability, coordinated solvent and selected $C$ atoms drawn capped stick, peripheral $C \& O$ atoms drawn wireframe. Tetraethylammonium cations, all $\mathrm{H}$ atoms and two pyridine lattice solvent molecules omitted for clarity. Selected bond distances $(\AA ̊)$ and angles $\left({ }^{\circ}\right)$ for 1*-p-Ad: Ce1-01 2.231(2), Ce1-02 2.209(2), Ce1-O3 2.151(3), Ce1-04 2.154(3), Ce1-O5 2.168(3), Ce1-N1 2.659(3), O1-Ce1-O2 85.35(9), O1-Ce1-O3 87.96(10), O2-Ce1-O4 98.58(10), O2-Ce1-O5 90.47(10), O3-Ce1O4 98.45(11), O3-Ce1-N1 79.46(11). 
Table S6: Crystallographic data for $\mathbf{1}^{*}$-p-Ad.

\begin{tabular}{|c|c|}
\hline \multicolumn{2}{|l|}{ Crystal data } \\
\hline CCDC deposition no. & 2032589 \\
\hline Chemical formula & $\mathrm{C}_{104} \mathrm{H}_{150} \mathrm{Ce}_{2} \mathrm{~N}_{2} \mathrm{O}_{10} \mathrm{Si}_{6} \cdot 2\left(\mathrm{C}_{8} \mathrm{H}_{20} \mathrm{~N}\right) \cdot 4\left(\mathrm{C}_{5} \mathrm{H}_{5} \mathrm{~N}\right)$ \\
\hline$M_{\mathrm{r}}$ & 2613.93 \\
\hline $\begin{array}{l}\text { Crystal system, } \\
\text { space group }\end{array}$ & Triclinic, $P 1$ \\
\hline Temperature $(\mathrm{K})$ & 190 \\
\hline$a, b, c(\AA)$ & 14.1625 (7), 14.4859 (7), 19.8390 (8) \\
\hline$\alpha, \beta, \gamma\left(^{\circ}\right)$ & $97.975(4), 105.839(4), 112.170(4)$ \\
\hline$V\left(\AA^{3}\right)$ & $3490.0(3)$ \\
\hline$Z$ & 1 \\
\hline Radiation type & Mo Ka \\
\hline$\mu\left(\mathrm{mm}^{-1}\right)$ & 0.75 \\
\hline Crystal size $(\mathrm{mm})$ & $0.19 \times 0.14 \times 0.08$ \\
\hline \multicolumn{2}{|l|}{ Data collection } \\
\hline Diffractometer & Xcalibur, Eos \\
\hline $\begin{array}{l}\text { Absorption } \\
\text { correction }\end{array}$ & $\begin{array}{l}\text { Analytical } \\
\text { CrysAlis PRO 1.171.40.54a (Rigaku Oxford Diffraction, 2019) Analytical numeric absorption correction using a } \\
\text { multifaceted crystal model based on expressions derived by R.C. Clark \& J.S. Reid. (Clark, R. C. \& Reid, J. S. } \\
\text { (1995). Acta Cryst. A51, 887-897) Empirical absorption correction using spherical harmonics, implemented in } \\
\text { SCALE3 ABSPACK scaling algorithm. }\end{array}$ \\
\hline$T_{\min ,} T_{\max }$ & $0.805,0.896$ \\
\hline $\begin{array}{l}\text { No. of measured, } \\
\text { independent and } \\
\text { observed }[I>2 \sigma(I)] \\
\text { reflections }\end{array}$ & $66835,14152,11546$ \\
\hline$R_{\text {int }}$ & 0.084 \\
\hline$(\sin \theta / \lambda)_{\max }\left(\AA^{-1}\right)$ & 0.625 \\
\hline \multicolumn{2}{|l|}{ Refinement } \\
\hline $\begin{array}{l}R\left[F^{2}>\right. \\
\left.2 \sigma\left(F^{2}\right)\right], w R\left(F^{2}\right), S\end{array}$ & $0.054,0.123,1.08$ \\
\hline No. of reflections & 14152 \\
\hline No. of parameters & 763 \\
\hline $\mathrm{H}$-atom treatment & $\mathrm{H}$-atom parameters constrained \\
\hline$\Delta \rho_{\max }, \Delta \rho_{\min }\left(\mathrm{e} \AA^{-3}\right)$ & $1.69,-0.80$ \\
\hline
\end{tabular}

\section{S5. References}


1. Wells, J. A. L.; Seymour, M. L.; Suvova, M.; Arnold, P. L., Dinuclear Uranium Complexation and Manipulation Using Robust Tetraaryloxides. Dalton Trans. 2016, 45 (40), 16026-16032.

2. Arnold, P. L.; Cowie, B. E.; Halliday, C. J. V., Isolation of f-Block Cation Pairs by Stretched Dinucleating Aryloxide Platforms. manuscript in preparation.

3. Gademann, K. C., David E.; Jacobsen, Eric N., Highly Enantioselective Inverse-ElectronDemand Hetero-Diels-Alder Reactions of $\alpha, \beta$-Unsaturated Aldehydes. Angew. Chem. Int. Ed. 2002, 41 (No. 16), 3059-3061.

4. Mansell, S. M.; Perandones, B. F.; Arnold, P. L., New UIII and U'v Silylamides and an Improved Synthesis of $\mathrm{NaN}\left(\mathrm{SiMe}_{2} \mathrm{R}\right)_{2}(\mathrm{R}=\mathrm{Me}$, Ph). J. Organomet. Chem. 2010, 695 (25-26), 2814-2821.

5. Loble, M. W.; Keith, J. M.; Altman, A. B.; Stieber, S. C.; Batista, E. R.; Boland, K. S.; Conradson, S. D.; Clark, D. L.; Lezama Pacheco, J.; Kozimor, S. A.; Martin, R. L.; Minasian, S. G.; Olson, A. C.; Scott, B. L.; Shuh, D. K.; Tyliszczak, T.; Wilkerson, M. P.; Zehnder, R. A., Covalency in Lanthanides. An X-ray Absorption Spectroscopy and Density Functional Theory Study of $\operatorname{LnCl}_{6}(\mathrm{x}-)(\mathrm{x}=$ 3, 2). J. Am. Chem. Soc. 2015, 137 (7), 2506-23.

6. Cetinkaya, B.; Gumrukcu, I.; Lappert, M. F., Lithium and Sodium 2,6-Di- tert-butylphenoxides and the Crystal and Molecular Structure of $\left[\mathrm{Li}\left(\mathrm{OC}_{6} \mathrm{H}_{2} \mathrm{Me}-4-\mathrm{Bu}, 2-2,6\right)\left(\mathrm{OEt}_{2}\right)\right]_{2}$. J. Am. Chem. Soc. 1980, 102 (6), 2086-2088.

7. Chen, H.-Y.; Mialon, L.; Abboud, K. A.; Miller, S. A., Comparative Study of Lactide Polymerization with Lithium, Sodium, Magnesium, and Calcium Complexes of BHT. Organometallics 2012, 31 (15), 5252-5261.

8. $\quad$ Friedrich, J.; Maichle-Mössmer, C.; Schrenk, C.; Schnepf, A.; Anwander, R., Ceric Ammonium Nitrate and Ceric Ammonium Chloride as Precursors for Ceric Siloxides: Ammonia and Ammonium Inclusion. Eur. J. Inorg. Chem. 2019, 19 (1), 79-90.

9. $\quad$ Gradeff, P. S.; Yunlu, K.; Deming, T. J.; Olofson, J. M.; Doedens, R. J.; Evans, W. J., Synthesis of Yttrium and Lanthanide Silyloxy Complexes from Anhydrous Nitrate and Oxo Alkoxide Precursors and the X-ray Crystal Structure of [Ce(OSiPh3)3(THF)3](THF). Inorg Chem 1990, 29, 420-424.

10. Garden, J. A.; Saini, P. K.; Williams, C. K., Greater than the Sum of Its Parts: A Heterodinuclear Polymerization Catalyst. J. Am. Chem. Soc. 2015, 137 (48), 15078-81.

11. Romain, C.; Garden, J. A.; Trott, G.; Buchard, A.; White, A. J. P.; Williams, C. K., Di-Zinc-Aryl Complexes: $\mathrm{CO}_{2}$ Insertions and Applications in Polymerisation Catalysis. Chemistry 2017, 23 (30), 7367-7376.

12. Jeon, J. Y.; Eo, S. C.; Varghese, J. K.; Lee, B. Y., Copolymerization and Terpolymerization of Carbon Dioxide/Propylene Oxide/Phthalic Anhydride Using a (salen)Co(III) Complex Tethering Four Quaternary Ammonium Salts. Beilstein J. Org. Chem. 2014, 10, 1787-95.

13. Longo, J. M.; DiCiccio, A. M.; Coates, G. W., Poly(propylene succinate): A New Polymer Stereocomplex. J. Am. Chem. Soc. 2014, 136 (45), 15897-900.

14. Isnard, F.; Santulli, F.; Cozzolino, M.; Lamberti, M.; Pellecchia, C.; Mazzeo, M., Tetracoordinate Aluminum Complexes Bearing Phenoxy-Based Ligands as Catalysts for Epoxide/Anhydride Copolymerization: some Mechanistic Insights. Catal. Sci. Technol. 2019, 9 (12), 3090-3098.

15. Peña Carrodeguas, L.; Martín, C.; Kleij, A. W., Semiaromatic Polyesters Derived from Renewable Terpene Oxides with High Glass Transitions. Macromolecules 2017, 50 (14), 5337-5345. 16. Martinez de Sarasa Buchaca, M.; de la Cruz-Martinez, F.; Martinez, J.; Alonso-Moreno, C.; Fernandez-Baeza, J.; Tejeda, J.; Niza, E.; Castro-Osma, J. A.; Otero, A.; Lara-Sanchez, A., Alternating Copolymerization of Epoxides and Anhydrides Catalyzed by Aluminum Complexes. ACS Omega 2018, 3 (12), 17581-17589.

17. Trott, G.; Garden, J. A.; Williams, C. K., Heterodinuclear Zinc and Magnesium Catalysts for Epoxide $/ \mathrm{CO}_{2}$ Ring Opening Copolymerizations. Chem. Sci. 2019, 10 (17), 4618-4627.

18. Saini, P. K.; Romain, C.; Zhu, Y.; Williams, C. K., Di-Magnesium and Zinc Catalysts for the Copolymerization of Phthalic Anhydride and Cyclohexene Oxide. Polym. Chem. 2014, 5 (20), 60686075. 
19. Sheldrick, G. M., SHELXT - Integrated Space-Group and Crystal-Structure Determination. Acta Cryst. 2015, A71, 3-8.

20. Sheldrick, G. M., Crystal Structure Refinement with SHELXL. Acta Cryst. 2015, C71, 3-8.

21. Dolomanov, O. V.; Bourhis, L. J.; Gildea, R. J.; Howard, J. A. K.; Puschmann, H., OLEX2: A Complete Structure Solution, Refinement and Analysis Program. J. Appl. Crystallogr. 2009, 42 (2), 339-341. 